Ricardo Cesare Roman Amigo

\title{
Design of Adsorption Systems by Topology Optimisation
}




\section{Ricardo Cesare Roman Amigo}

\section{Design of Adsorption Systems by Topology Optimisation}

Submitted to the Escola Politécnica of the Universidade de São Paulo in partial fulfilment of the requirements for the degree of Doutor em Ciências.

Área de Concentração:

Control and Mechanical Automation Engineering

Orientador:

Prof. Dr. Emilio Carlos Nelli Silva

Co-orientador:

Dr. Robert William Hewson 
Autorizo a reprodução e divulgação total ou parcial deste trabalho, por qualquer meio convencional ou eletrônico, para fins de estudo e pesquisa, desde que citada a fonte.

Este exemplar foi revisado e corrigido em relação à versão original, sob responsabilidade única do autor e com a anuência de seu orientador.

São Paulo, de de

Assinatura do autor:

Assinatura do orientador:

\section{Catalogação-na-publicação}

Amigo, Ricardo Cesare Roman

Projeto de Sistemas de Adsorção por Otimização Topológica / R. C. R. Amigo -- versão corr. -- São Paulo, 2018. $118 \mathrm{p}$.

Tese (Doutorado) - Escola Politécnica da Universidade de São Paulo. Departamento de Engenharia Mecatrônica e de Sistemas Mecânicos.

1.Topologia (Otimização) 2.Método dos Elementos Finitos 3.Adsorção 4.Combustíveis Gasosos I.Universidade de São Paulo. Escola Politécnica. Departamento de Engenharia Mecatrônica e de Sistemas Mecânicos II.t. 
Ao meu pai. 


\section{Acknowledgments}

I would like to thank my supervisors Dr. Rob Hewson and Prof. Emilio Silva for their help and guidance over the past years. I would also like to thank Dr. José Paiva for his important contributions to this work.

In addition, I gratefully acknowledge the support of the RCGI Research Centre for Gas Innovation, sponsored by FAPESP (2014/50279-4) and Shell, and CNPq (National Council for Research and Development), under grants 140664/2013-0 and 200404/2014-8.

I would like to thank my research friends at USP and at Imperial for their essential and intangible contribution to this doctorate, especially on the non-academic side. Naturally, I must extend my thanks to London and all the great people I have met there for some of the best days of my life.

Finalmente, agradeço à minha nonna, à minha mãe, às minhas irmãs Carolina e Cristina, e ao meu cachorrão Kepler, pelo essencial apoio que me deram à distância durante este trabalho. 


\section{Resumo}

Adsorção é um mecanismo de retençao de moléculas de um fluido em superfícies solidas e apresenta um vasto campo de aplicações, como em refrigeração, processos de separação e armazenamento de combustível. Este trabalho descreve o modelo de adsorção de gás em meios porosos e define uma abordagem de otimização de sistemas de adsorção baseada no Método de Otimização Topológica (MOT). Uma formulação abrangente para os fenômenos de adsorção e dessorção é apresentada, detalhando a dedução de seus formas fracas e montagem das matrizes para a implementação do Método dos Elementos Finitos (MEF). As sensibilidades das funções-objetivo em relação às variaveis de projeto são obtidas pelo método adjunto, que também é apresentado em detalhe. A implementação numerica é auxiliada pelos pacotes FEniCS e Dolfin Adjoint, originando uma ferramenta genérica para a otimização de sistemas de adsorção. Este método é aplicado ao projeto de tanques de Gas Natural Adsorvido (GNA) visando transporte, armazenamento e consumo. Tanques otimizados são propostos e comparados com tanques tradicionais, apresentando melhora dos objetivos de projeto.

Palavras-Chave - Topologia (Otimização), Método dos Elementos Finitos, Adsorção. 


\section{Abstract}

Adsorption is a retention mechanism of fluid molecules on solid surfaces and presents a wide range of applications, such as refrigeration, separation processes and fuel storage. This work describes the modelling of gas adsorption on porous media and presents an optimisation approach for the design of adsorption systems based on topology optimisation. A comprehensive formulation for the adsorption and desorption phenomena is presented, detailing the derivation of their weak forms and assembling the matrices for the implementation of the Finite Element Method (FEM). The sensitivities of objective functions to topology optimisation design variables are obtained by the adjoint method, which is presented in detail. The numerical implementation is aided by FEniCS and Dolfin Adjoint packages, resulting in a generic software for the optimisation of adsorption systems. The method is applied for the design of Adsorbed Natural Gas (ANG) tanks for on-demand consumption. Optimised tank layouts are proposed and compared with standard tanks, presenting improvement of the design objectives.

Keywords - Topology Optimisation, Finite Elements Method, Adsorption. 


\section{List of Figures}

1.1 Distribution of molecules in gaseous (o) and adsorbed $(\bullet)$ phases. . . . . . 2

1.2 Adsorption cooling process. . . . . . . . . . . . . . . 3

1.3 Topology optimisation procedure. . . . . . . . . . . . . . . . . 10

2.1 Continuum modelling of porous media based on Representative Elementary Volume $(\mathrm{REV}) . \ldots \ldots \ldots \ldots \ldots$. . . . . . . . . . . . . . . . . . . . . . . . .

2.2 Adsorption and desorption processes. . . . . . . . . . . . . . . 14

2.3 Adsorption energetics. . . . . . . . . . . . . . . . 15

2.4 Classes of isotherms. . . . . . . . . . . . . . . . 16

2.5 Adsorption domain. . . . . . . . . . . . . . . . . . . . . 19

2.6 Inlet pressure $\left(P_{\Gamma_{L}}\right)$ during (a) adsorption and (b) desorption cycles. . . . 23

3.1 Optimisation of ANG tanks through the addition of non-adsorbent materials. 29

3.2 Isomassic curves given by the variation of adsorbent temperature and relative non-adsorbent volume in adsorption cycle. . . . . . . . . . . . . 30

3.3 Cycle considered for the optimisation of ANG tanks applied to on-demand consumption. . . . . . . . . . . . . . . . . . 31

3.4 SIMP for Steel and Carbon properties. . . . . . . . . . . . . . 34

3.5 Borrvall's material model for Steel and Carbon Permeability $(K)$. . . . . 35

3.6 Hyperbolic tangent material model for Steel and Carbon properties with

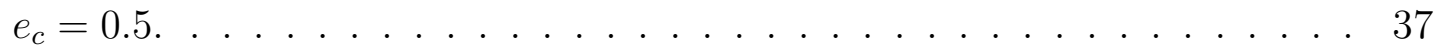

3.7 Material model for properties A and B. . . . . . . . . . . . . . 38

4.1 Custom axisymmetric tank domain (SAHOO et al., 2011) . . . . . . . . . 47

4.2 Flow rate of $1 \mathrm{~L} \mathrm{~min}^{-1}$ : Comparison with the experimental results in (SAHOO et al., 2011). . . . . . . . . . . . . . . . . . . . 47

4.3 Flow rate of $10 \mathrm{~L} \mathrm{~min}^{-1}$ : Comparison with the experimental results in (SAHOO et al., 2011). . . . . . . . . . . . . . . . . . . . . . 48 
4.4 Flow rate of $30 \mathrm{~L} \mathrm{~min}^{-1}$ : Comparison with the experimental results in (SAHOO et al., 2011). . . . . . . . . . . . . . . . . . . . . . . 48

4.5 Temperature distribution at the end of the adsorption cycle. . . . . . . . . 49

4.6 Adsorption domain adopted for verifying the sensitivity of adsorption cycles to the smoothness factor $\sigma \ldots \ldots \ldots \ldots$. . . . . . . . . . 50

4.7 Total mass of gas along the adsorption cycle for different smoothness factors. 50

4.8 Smoothness factor convergence. . . . . . . . . . . . . . . 51

4.9 Adsorption domain constituted by Activated Carbon and Steel. . . . . . . 51

4.10 Pressure distribution on Carbon-Steel domain. . . . . . . . . . . . . . . 52

4.11 Adsorption domain adopted for verifying mesh and timestep convergence. . 53

4.12 Mesh convergence. . . . . . . . . . . . . . . . 53

4.13 Timestep convergence. . . . . . . . . . . . . . . . 54

4.14 Methods for obtaining the adjoint code (FUNKE, 2012) . . . . . . . . 55

4.15 Optimisation activity diagram. . . . . . . . . . . . 57

4.16 Original (top) and Perturbed Pseudo-Porosity fields for (from the top) $h=1.00 \times 10^{-3}, h=5.00 \times 10^{-4}, h=2.50 \times 10^{-5}, h=1.25 \times 10^{-5}$, $h=6.25 \times 10^{-6} \ldots \ldots \ldots \ldots \ldots \ldots$

5.1 Pseudo-porosity fields evolution (top-down) for SIMP (left), non-offset hyperbolic tangents (centre) and offset hyperbolic tangents (right) material models for prismatic tank optimisation. . . . . . . . . . . . 66

5.2 Functional convergence for different material models. . . . . . . . . . . 67

5.3 Pseudo-porosity fields evolution for homogeneous (left) and arbitrary (right) initial guesses for prismatic tank optimisation starting with $p_{i n i}=1$. . . .

5.4 Pseudo-porosity fields evolution for homogeneous (top image on each block) and arbitrary (bottom) initial guesses for cylindrical tank optimisation starting with $p_{i n i}=20 \ldots \ldots \ldots \ldots \ldots$

5.5 Pseudo-porosity fields evolution for homogeneous (top image on each block) and arbitrary (bottom) initial guesses for cylindrical tank optimisation starting with $p_{i n i}=1 \ldots \ldots \ldots \ldots \ldots$ 
5.6 Optimised design for a cylindrical domain subjected to natural convection. 75

5.7 Optimised design for a cylindrical domain with non-adsorbent material with high thermal conductivity. . . . . . . . . . . . . . . . . . 77

5.8 Optimised design for a cylindrical domain with non-adsorbent material with high heat capacity. . . . . . . . . . . . . . . . . . . . 80

5.9 Optimised design for a cylindrical domain with non-adsorbent material with high heat capacity in 3600 s filling cycle. . . . . . . . . . . . . . . . 81

5.10 Optimised design for a cylindrical domain with non-adsorbent material with high heat capacity in 1200 s filling cycle. . . . . . . . . . . . . . . 83

5.11 Optimised design for a prismatic domain subjected to forced convection. . 85

5.12 Optimised design for a custom axisymmetric domain subjected to forced convection with $h=100 \mathrm{Wm}^{-2} \mathrm{~K}^{-1} \ldots \ldots \ldots \ldots 8$

5.13 Optimised design for a custom axisymmetric domain subjected to forced convection with $h=700 \mathrm{Wm}^{-2} \mathrm{~K}^{-1} \ldots \ldots \ldots . \ldots . \ldots . \ldots 89$

5.14 Optimised design for a cylindrical domain with forced convection. . . . . . 91

5.15 Optimised design for a cylindrical domain with forced convection in $10800 \mathrm{~s}$ filling cycle. . . . . . . . . . . . . . . . . . . . . . 93

5.16 Optimised design for a cylindrical domain with forced convection in $3600 \mathrm{~s}$ filling cycle. . . . . . . . . . . . . . . . . . . . . . . 9 95

5.17 Optimised design for a cylindrical domain with natural convection and cold finger. . . . . . . . . . . . . . . . . . . . 96

5.18 Optimised design for a small prismatic domain subjected to forced convection. 99

5.19 Optimised design for a large prismatic domain subjected to forced convection. 100

5.20 Optimised design for a custom axisymmetric domain subjected to $3 \mathrm{MPa}$ filling pressure and forced convection with $h=100 \mathrm{Wm}^{-2} \mathrm{~K}^{-1}$. . . . . . 102

5.21 Optimised design for a custom axisymmetric domain subjected to $5 \mathrm{MPa}$ filling pressure and forced convection with $h=100 \mathrm{Wm}^{-2} \mathrm{~K}^{-1}$. . . . . . 103 


\section{List of Tables}

4.1 Taylor Remainder Convergence. . . . . . . . . . . . . . . . . . . . . . 59

5.1 Standard domain and cycle for prismatic tanks. . . . . . . . . . . . 61

5.2 Standard domain and cycle for cylindrical tanks. . . . . . . . . . . . . 62

5.3 Standard domain and cycle for a custom axisymmetric tank. . . . . . . . 63

5.4 Optimisation definitions for a prismatic tank subjected to forced convection. 65

5.5 Optimisation definitions for a prismatic tank subjected to forced convection. 68

5.6 Optimisation definitions for a cylindrical domain with non-adsorbent material with high heat capacity. . . . . . . . . . . . . . . . . 70

5.7 Optimisation definitions for a cylindrical domain subjected to natural con-

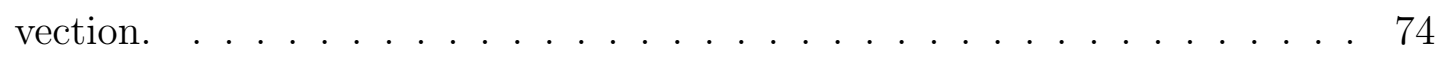

5.8 Optimisation definitions for a cylindrical domain with non-adsorbent material with high thermal conductivity. . . . . . . . . . . . . . 76

5.9 Optimisation definitions for a cylindrical domain with non-adsorbent material with high heat capacity. . . . . . . . . . . . . . . . 79

5.10 Optimisation definitions for a cylindrical domain with non-adsorbent material with high heat capacity in $3600 \mathrm{~s}$ filling cycle. . . . . . . . . . . . 79

5.11 Optimisation definitions for a cylindrical domain with non-adsorbent material with high heat capacity in $1200 \mathrm{~s}$ filling cycle. . . . . . . . . . . . 82

5.12 Optimisation definitions for a prismatic domain subjected to forced con-

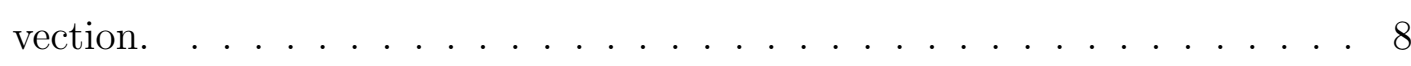

5.13 Optimisation definitions for a custom axisymmetric domain subjected to forced convection with $h=100 \mathrm{Wm}^{-2} \mathrm{~K}^{-1} \ldots \ldots \ldots$. . . . . . 87

5.14 Optimisation definitions for a custom axisymmetric domain subjected to forced convection with $h=700 \mathrm{Wm}^{-2} \mathrm{~K}^{-1} \ldots \ldots \ldots$. . . . . . 87

5.15 Optimisation definitions for a custom axisymmetric domain subjected to natural convection and connected to a cold finger. . . . . . . . . . . . . . 90 
5.16 Optimisation definitions for a cylindrical domain with forced convection in 10800 s filling cycle. . . . . . . . . . . . . . . . . . . . 92

5.17 Optimisation definitions for a cylindrical domain with forced convection in 3600 s filling cycle. . . . . . . . . . . . . . . . . . . . . 94

5.18 Optimisation definitions for a cylindrical domain subjected to natural convection and connected to a cold finger. . . . . . . . . . . . . . . . . . . . 94

5.19 Optimisation definitions for a small prismatic domain subjected to forced convection. . . . . . . . . . . . . . . . . . . . . 98

5.20 Optimisation definitions for a small prismatic domain subjected to forced convection. . . . . . . . . . . . . . . . . . . . . 98

5.21 Optimisation definitions for a custom axisymmetric domain subjected to $3.00 \mathrm{MPa}$ filling pressure and forced convection with $h=100 \mathrm{Wm}^{-2} \mathrm{~K}^{-1} \ldots 101$

5.22 Optimisation definitions for a custom axisymmetric domain subjected to $5.00 \mathrm{MPa}$ filling pressure and forced convection with $h=100 \mathrm{Wm}^{-2} \mathrm{~K}^{-1} \ldots 101$

B.1 Methane properties (SAHOO et al., 2011; SAHOO; RAMGOPAL, 2014). . 117

B.2 Activated Carbon properties (SAHOO et al., 2011) . . . . . . . . . . . 117

B.3 Adsorption parameters for Methane on Activated Carbon (SAHOO et al., 2011; SAHOO; RAMGOPAL, 2014) . . . . . . . . . . . . . . . 118

B.4 Steel properties. . . . . . . . . . . . . . . . . . . . 118 


\section{Acronyms}

$\begin{array}{ll}\text { ANG } & \text { Adsorbed Natural Gas } \\ \text { CCS } & \text { CO } 2 \text { Capture and Storage } \\ \text { CNG } & \text { Compressed Natural Gas } \\ \text { DA } & \text { Dubinin-Astakhov } \\ \text { DR } & \text { Dubinin-Radushevich } \\ \text { ECP } & \text { Element Connectivity Parametrisation } \\ \text { ESO } & \text { Evolutionary Structural Optimisation } \\ \text { ETM } & \text { Electro-Thermo-Mechanical Actuator } \\ \text { FEM } & \text { Finite Element Method } \\ \text { FFC } & \text { FEniCS Form Compiler } \\ \text { FFD } & \text { Free-Form Deformation } \\ \text { FIAT } & \text { FEniCS Finite Element Automatic Tabulator } \\ \text { FVM } & \text { Finite Volume Method } \\ \text { GITT } & \text { Generalised Integral Transform Technique } \\ \text { GWP } & \text { Global Warming Potential } \\ \text { L-BFGS-B } & \text { Limited Memory Broyden-Fletcher-Goldfarb-Shanno with Box Constraints } \\ \text { LDF } & \text { Linear Driving Force Model } \\ \text { LNG } & \text { Liquefied Natural Gas } \\ \text { MOF } & \text { Metal-Organic Framework } \\ \text { ODE } & \text { Ordinary Differential Equation } \\ \text { OHT } & \text { Offset Hyperbolic Tangents Material Model } \\ \text { PCM } & \text { Phase Change Material } \\ \text { PDE } & \text { Partial Differential Equation } \\ \text { PLIC } & \text { Piecewise Linear Interface Calculation } \\ \text { PSA } & \text { Pressure Swing Adsorption } \\ \text { RBF } & \text { Radial Basis Function } \\ \text { REV } & \text { Representative Elementary Volume } \\ \text { SIMP } & \text { Solid Isotropic Material with Penalisation } \\ \text { TSA } & \text { Temperature Swing Adsorption } \\ \text { UFC } & \text { FEniCS Unified Form-Assembly Code } \\ \text { VoF } & \text { Volume of Fluid } \\ \end{array}$




\section{Notation}

$A \quad$ Polanyi's adsorption potential

$C \quad$ Solute concentration

$C p_{g} \quad$ Specific heat capacity of gaseous phase

$C p_{s} \quad$ Specific heat capacity of solid phase

$D \quad$ Intra-particle diffusional time constant

$\mathbb{D} \quad$ Adsorption kinetics weak form

$D_{0} \quad$ Diffusivity of gaseous phase

e Pseudo-porosity

\& $\quad$ Non-filtered pseudo-porosity

$\mathbb{E} \quad$ Energy balance weak form

$E_{0} \quad$ Characteristic energy of adsorption

$E_{a} \quad$ Activation energy of adsorption

$E_{d} \quad$ Activation energy of desorption

$G \quad$ Kinetic coefficient of adsorption and desorption reactions

$h_{W} \quad$ Heat convection coefficient on external walls

$i t_{\max } \quad$ Maximum iterations per continuation step

$\Delta H \quad$ Isosteric heat of adsorption

$J \quad$ Functional of interest

$k_{a} \quad$ Kinetic coefficient of adsorption

$k_{d} \quad$ Kinetic coefficient of desorption

$k_{f} \quad$ Thermal conductivity of phases combined

$k_{g} \quad$ Thermal conductivity of gaseous phase

$k_{\text {intra }} \quad$ Intra-particle diffusion rate constant

$k_{s} \quad$ Thermal conductivity of solid phase

$K_{s} \quad$ Permeability of the solid phase

$\mathbb{M} \quad$ Continuity weak form

$\dot{m}_{\text {let }} \quad$ Mass flow

$M_{g} \quad$ Molecular mass of gaseous phase

$n_{s} \quad$ Micro-pores dispersion

$p \quad$ Material model penalisation

$P \quad$ Pressure

$\mathbb{P} \quad$ Generic material property

$P_{a t m} \quad$ Atmospheric pressure

$P_{c r} \quad$ Critical pressure

$P_{l e t} \quad$ Pressure at inlet/outlet regions

$P_{\text {sat }} \quad$ Saturated pressure

$q \quad$ Concentration of adsorbed phase

$Q \quad$ Density of adsorbed phase

$q_{e q} \quad$ Equilibrium concentration of adsorbed phase

$Q_{e q} \quad$ Equilibrium density of adsorbed phase

$r \quad$ Radial coordinate in axisymmetric domains

$R_{g} \quad$ Universal gas constant

$T \quad$ Temperature 


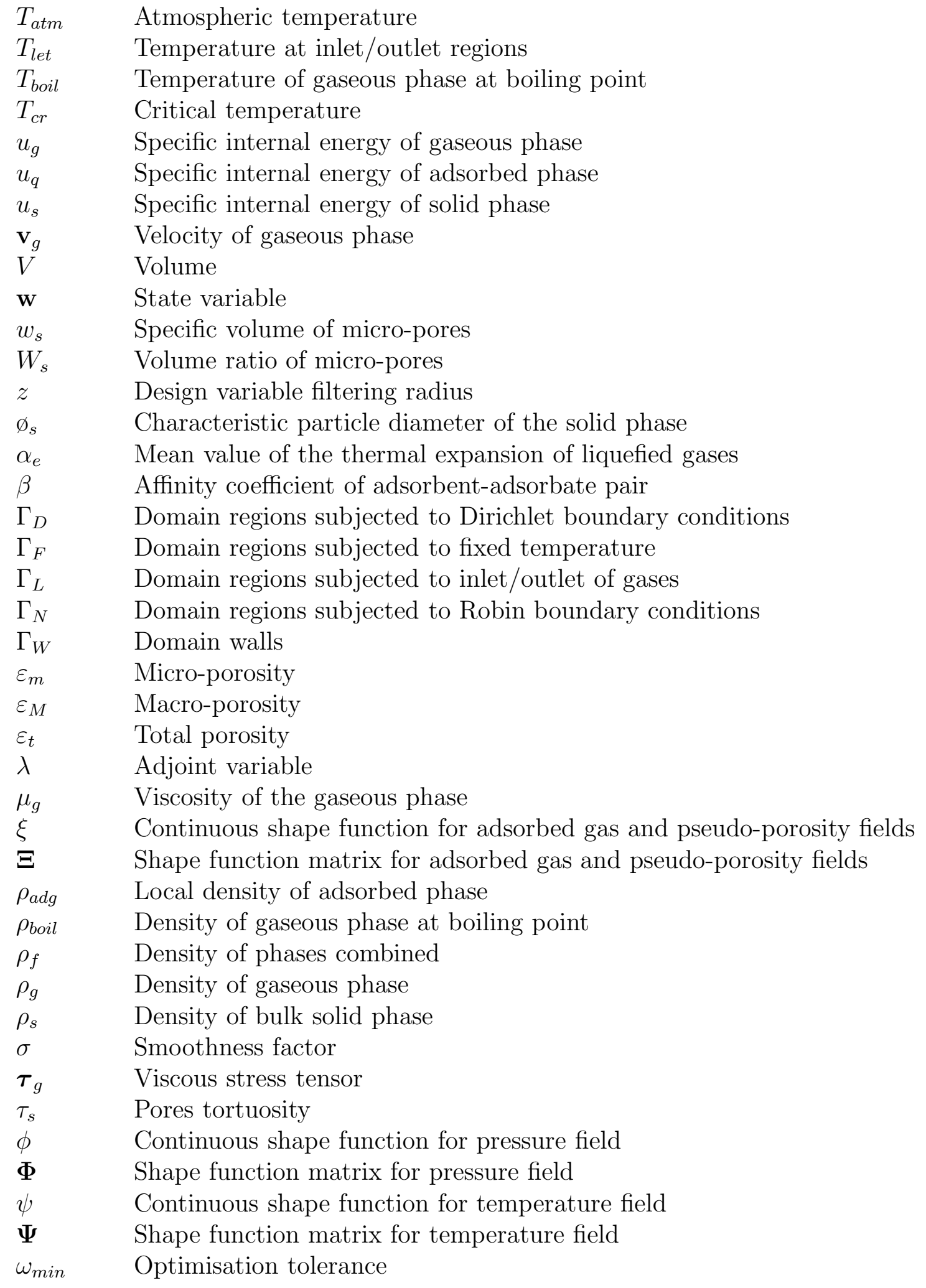




\section{Contents}

1 Introduction 1

1.1 Concept and Applications of the Adsorption Phenomenon . . . . . . . . 1

1.2 Adsorbed Natural Gas Tanks . . . . . . . . . . . . . . . . . . . . . 4

1.2.1 Design Approaches ................... . . 5

1.2.2 Numerical Modelling . . . . . . . . . . . . . . . . 6

1.3 Optimisation Methods applied to Fluids and Heat Transfer Problems . . . 7

1.4 Motivation . . . . . . . . . . . . . . . . . . 10

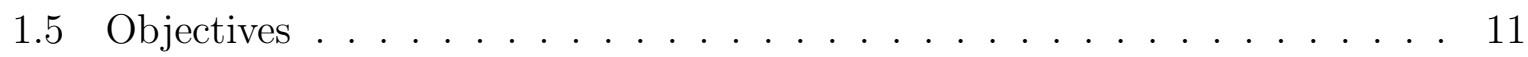

1.6 Scientific Contributions . . . . . . . . . . . . . . . . . . . 11

1.7 Thesis Outline . . . . . . . . . . . . . . . . . . . . 11

2 Modelling of Adsorption Systems $\quad 12$

2.1 Modelling Flow in Porous Media . . . . . . . . . . . . . . . . . 12

2.2 Modelling Adsorption Equilibrium and Kinetics . . . . . . . . . . . . . . . 14

2.3 Governing Equations . . . . . . . . . . . . . . . . . . . . . . . . 18

2.3 .1 Natural Material Models . . . . . . . . . . . . . . . . . . 20

2.3.2 Adsorption and Desorption Kinetics . . . . . . . . . . . . . . 20

2.3 .3 Continuity . . . . . . . . . . . . . . . . 22

2.3.4 Energy Balance . . . . . . . . . . . . . . . . . 23

2.4 Weak Formulation of Adsorption Systems . . . . . . . . . . . . 25

3 Optimisation of ANG Tanks 28

3.1 Design Concept . . . . . . . . . . . . . . . . . . . 28

3.2 Manufacturing Considerations . . . . . . . . . . . . . . . . . . . . . 29 
3.3 Objective Function . . . . . . . . . . . . . . . . . . . 31

3.4 Material Model . . . . . . . . . . . . . . . . . . . . . . . . . 32

3.4.1 Material Models with Penalisation . . . . . . . . . . . . . . 32

3.4.2 Offset Hyperbolic Tangents (OHT) Material Model . . . . . . . . . 33

4 Numerical Implementation and Verification 39

4.1 Discrete Adsorption Problem . . . . . . . . . . . . . . . . . . . 39

4.2 Discrete Adjoint System for the Adsorption Problem . . . . . . . . . . . . 41

4.3 Adsorption Problem Implementation . . . . . . . . . . . . . . . . . . . . 45

4.3.1 System Decoupling Verification . . . . . . . . . . . . . . . 45

4.3 .2 Pressure Loading Verification . . . . . . . . . . . . . . . . . 47

4.3 .3 Impermeability Verification _. . . . . . . . . . . . . . . . . . 49

4.3.4 Mesh and Timestep Convergence . . . . . . . . . . . . . . . 52

4.4 Topology Optimisation Implementation . . . . . . . . . . . . . . . . . 53

4.4.1 Design Variables Filtering . . . . . . . . . . . . . . 54

4.4 .2 Optimisation Algorithm . . . . . . . . . . . . . 56

4.4.3 Adjoint Sensitivites Verification . . . . . . . . . . . . . . . 57

5 Results and Discussion $\quad 60$

5.1 Definition of Standard Domains and Cycles for the Design of ANG Tanks . 60

5.2 Evaluation of the OHT Material Model for the Design of ANG Tanks . . . 64

5.2.1 OHT vs. Traditional Material Models . . . . . . . . . . . . . . . . 64

5.2.2 Dependency on the Initial Guess and Penalisation . . . . . . . . . . 68

5.3 Design of ANG Tanks with Passive Thermal Management . . . . . . . . . 70

5.3.1 Tanks Subjected to Natural Convection . . . . . . . . . . . . . . 73

5.3.2 Sensitivity to Thermal Conductivity . . . . . . . . . 76

5.3 .3 Sensitivity to Heat Capacity . . . . . . . . . . . . . . . . . . . 78

5.4 Design of ANG Tanks with Active Thermal Management . . . . . . . . . . 84 
5.4.1 Tanks Subjected to Forced Convection . . . . . . . . . . . . . . . . 84

5.4.2 Sensitivity to Forced Convection and Cold Walls . . . . . . . . . . . 86

5.4.3 Sensitivity to Shape Complexity . . . . . . . . . . . . . . . . . . 92

5.4.4 Sensitivity to Tank Dimensions . . . . . . . . . . . . . . . . 97

5.4.5 Sensitivity to Filling Pressure . . . . . . . . . . . . . . . . . 97

6 Conclusions

References

Appendix A - Finite Element Coefficients

A.1 Full Adsorption Problem . . . . . . . . . . . . . . . . . . 113

A.2 Adsorption Problem after Decoupling the Adsorbed Density Field . . . . . 114

A.3 Adsorption Problem after Decoupling the Pressure Field . . . . . . . . . . 115

A.4 Helmholtz Filtering . . . . . . . . . . . . . . . . . 116

Appendix B - Material Properties 


\section{Introduction}

In this Chapter, basic concepts of the adsorption phenomenon are introduced along with some applications, emphasizing Adsorbed Natural Gas (ANG) tanks. Thereafter, a brief review on optimisation methods applied to fluids and heat transfer problems is presented. Finally, the motivation, objectives and expected scientific contributions are stated, based on the lack of previous works approaching the design of adsorption systems by topology optimisation.

\subsection{Concept and Applications of the Adsorption Phe- nomenon}

The flow of gases or liquids in porous media are subjected to the retention and release of molecules on the solid phase. One of the retention mechanisms is the sorption phenomenon, which is a general term adopted for both adsorption and absorption reactions. The former is a surface reaction in which gas molecules accumulate on the surface of solid particles attracted by disrupted bonds (MOTA et al., 2004), while the latter is a bulk phenomenon, in which gas molecules diffuse through another phase, accumulating at the grain boundaries and often forming a new phase (BLANCO et al., 2014).

Adsorption is divided into two categories: (i) physical adsorption, based on weak van der Waals forces, which is also know as physisorption, and (ii) chemical adsorption, based on covalent bondings, also know as chemisorption. While the former is easily reversible and leaves the adsorbent intact, the latter involves a chemical reaction that affect the surfaces, as is the case of corrosion phenomenon. Figure 1.1 illustrates the mechanism of adsorption, where molecules concentrate on micro-pores walls.

The capacity of adsorption of porous media is dependent on characteristics such as the distribution and characteristic of micro-pores on the adsorbent, the affinity of adsorbent 


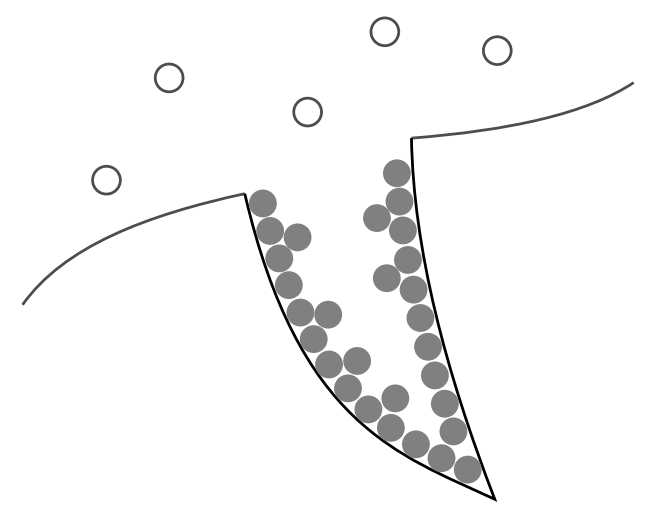

Figure 1.1: Distribution of molecules in gaseous (o) and adsorbed $(\bullet)$ phases.

and adsorbate, gas pressure and temperature (BILOE et al., 2002). Considering a given pair adsorbent-adsorbate, the capacity of adsorption increases as pressure increases and temperature decreases.

Several engineering applications are based on the adsorption process. For instance, it is widely employed for fluid separation, both for removing trace contaminants, which is known as purification processes, and for recovering components from a mixture, which is known as a bulk separation processes. Examples of purification are the separation of sulphurous compounds from natural gas, solvents from air and $\mathrm{SO}_{2}$ from vent streams (CAVALCANTE, 2000). Among bulk separations, the following pairs can be encountered: $\mathrm{N}_{2}-\mathrm{O}_{2}$, Water-Ethanol and Fructose-Glucose (CAVALCANTE, 2000).

The operation of adsorption separation processes can be undertaken as a batch process, by percolating fluid through the adsorbent until saturation and then purging it. The purge, also known as adsorbent regeneration, is usually undertaken by varying the temperature, which is termed Temperature Swing Adsorption (TSA), or by controlling the pressure, in a method called Pressure Swing Adsorption (PSA), or by purging the adsorbent with an inert desorbate, which could be easily separated by later processes, such as distillation. It is possible to have an adsorption separation in a continuous counter-current approach, by submitting adsorbents to adsorption and desorption at the same time, and also in a chromatographic way, which is based on the moving bed concept where the fluid flows in the opposite direction to the solid phase (CHARTON; NICOUD, 1995).

Adsorption can also be used for refrigeration, by using use solid adsorbents such as silica-gel and zeolite, to produce the cooling effect (WANG; VINEYARD, 2011). In this class of refrigerators, an adsorbent is dried out by applying heat, which increases its pressure and temperature and causes the desorption of refrigerant vapour. This step is equivalent to the compression step in compressor refrigerators. The vapour is then 
liquefied in the condenser, while heat is rejected to a cooling tower. When the adsorbent is sufficiently dried out, the heat intake stops and heat dissipation takes place. Next, the adsorbent connection switches from the condenser to the evaporator, where the saturated liquid boils, vapour is adsorbed and chilled water is generated, which is used for airconditioning. Given that this cycle is intermittent, continuous cooling output is achieved by associating two adsorbents driven anti-cyclically, as illustrated by Figure 1.2.

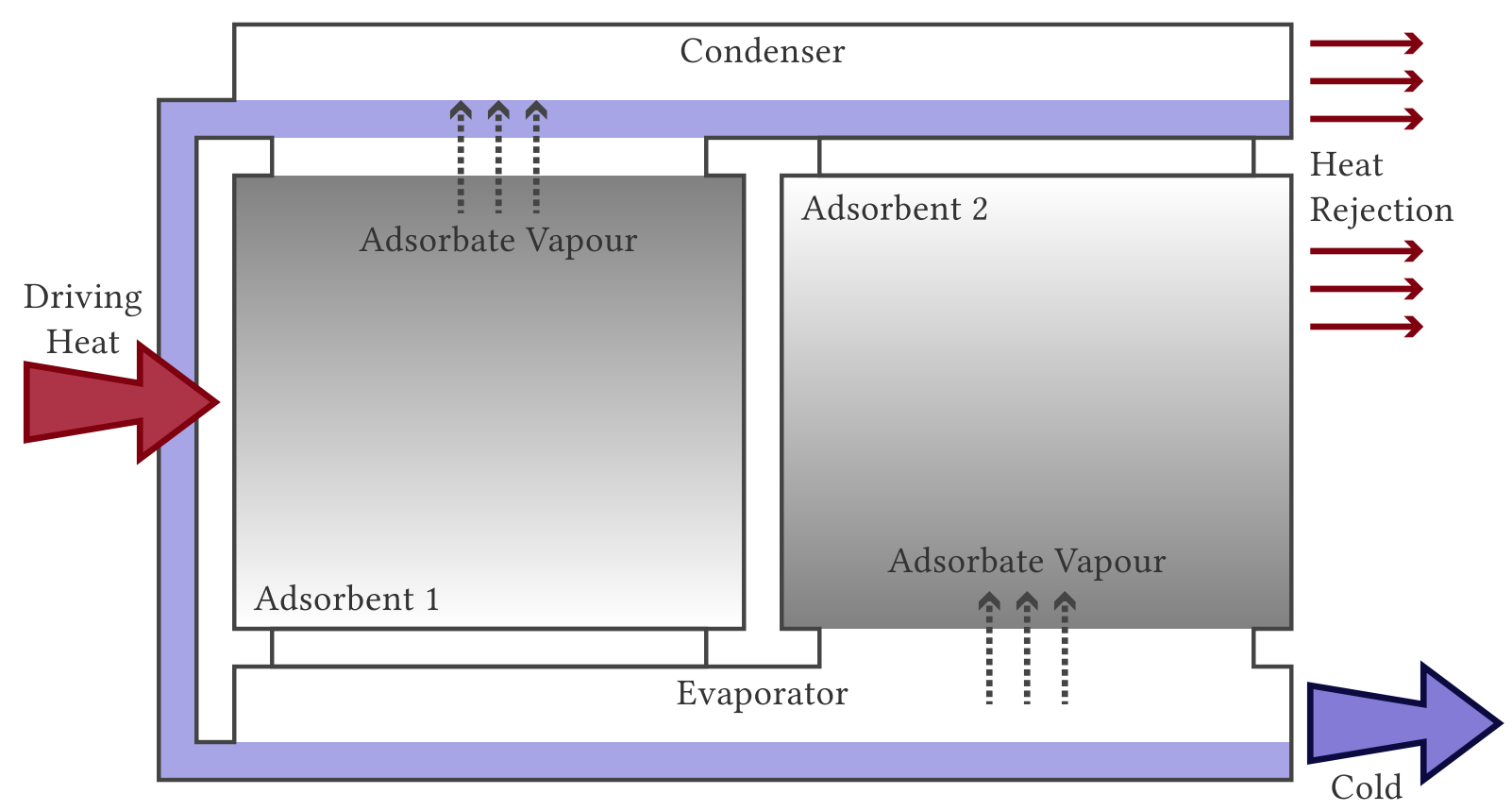

Generation

Figure 1.2: Adsorption cooling process (SOLARNEXT, 2009).

Adsorption systems present low electrical energy consumption in comparison to other refrigeration methods and can be activated by temperatures as low as $323 \mathrm{~K}$, while absorption systems require at least $363 \mathrm{~K}$ (WANG; VINEYARD, 2011). This opens up the possibility of using low-grade heat such that derived from solar energy. The refrigerants used in adsorption cycles have zero ozone depletion potential and low Global Warming Potential (GWP), which is an index dependent on the strength with which certain greenhouse gas absorbs infrared radiation, on the spectral location of its absorbing wavelengths and on its atmospheric lifetime (ELROD, 1999). They can also operate in environments with significant vibrations, since the adsorbent is solid (in contrast with the liquid used in absorbent refrigerators) and the systems have fewer mechanical parts. The main drawbacks of adsorbent refrigerators are their low coefficient of performance (quantity of achieved cooling effect per unit of heat delivered), intermittent operation (when not associated in two or more generators) and their large size and weight (WOLAK; KRASZEWSKI, 2016).

Adsorption is also promising in $\mathrm{CO}_{2}$ Capture and Storage (CCS). The greenhouse 
effect is drawing more and more attention from researchers worldwide. Although carbon dioxide naturally exists in the atmosphere, it is one of the greenhouse gases and its concentrations have increased due to human actions, such as burning fuels. In fact, according to Toledo et al. (2013), by the year 2100, CCS will be responsible for $15 \%$ to $55 \%$ of greenhouse gases reduction. One of the proposed methods for CCS consists in the capture of $\mathrm{CO}_{2}$ in installations where it can be placed for long-term storage in the earth crust. However, the pressure rise due to $\mathrm{CO}_{2}$ injection is a major concern regarding this technology. Finding a safe and effective method that can be feasible in industrial scale at competitive costs is the challenge regarding this topic. When it comes to $\mathrm{CO}_{2}$ capture, several solid adsorbents such as alumina, silicates, silica, silica-alumina, activated carbon, zeolites and also MOFs (Metal-Organic Frameworks) are known to be capable of adsorbing $\mathrm{CO}_{2}$. Aspects such as gas impurity $\left(\mathrm{CO}_{2}\right.$ mixed with other gases) (WETENHALL et al., 2014) and thermal behaviour hinders the operation of such systems and must be addressed during design process. These studies show not only adsorption systems can handle CCS but also they can be enhanced by adjusting characteristics of their geometry and composition. Optimizing such systems may bring more possibilities and capabilities for the CCS via adsorption.

Fuel storage also present a prolific area of application for adsorption. Given the low density of gas fuel, it is usual to store them in high-pressure or low-temperature vessels. In the case of natural gas storage, Compressed Natural Gas (CNG) is ordinarily stored at $200 \mathrm{~atm}$, offering a capacity of $245 \mathrm{~V} / \mathrm{V}$, which means a density 245 higher than at atmospheric temperature and pressure. In turn, Liquefied Natural Gas (LNG) requires cryogenic temperatures to force gas condensation, which happens, for methane, at around $113 \mathrm{~K}$ and provides a storage capacity of $600 \mathrm{~V} / \mathrm{V}$ (HIRATA et al., 2009). On the other hand, Adsorbed Natural Gas (ANG) tanks consist of natural gas adsorbed in a solid matrix of coal, accumulated in its pores. Due to the relatively high density of the adsorbed phase, an overall storage capacity around $164 \mathrm{~V} / \mathrm{V}$ can be achieved at room temperature with pressure ranging from $35 \mathrm{~atm}$ to $50 \mathrm{~atm}$ (HIRATA et al., 2009). These characteristics place ANG vessels as a promising method to maintain gas confinement, as it works at atmospheric temperature and much lower pressure then CNG.

\subsection{Adsorbed Natural Gas Tanks}

The ease of storing gas by the ANG method allows the expansion of the current applications of natural gas, both for just-in-time consumption and for long-term storage. 
The possibility of storing gas at lower pressure enables the adoption of wrappers made of lighter materials and with complex shapes, which would be prohibitive in high-pressure tanks due to stress concentration. The concept of box-shaped vessel for LNG storage (KAIST, 2014) can also be adopt in the ANG method, improving the efficiency in the use of space in comparison to cylindrical and spherical alternatives.

Supplying natural gas to consumption sites often present challenging logistics when the construction of a pipeline is not economically feasible. Gas supply is then guaranteed via virtual pipelines, which currently consist of trucks carrying CNG tanks to deliver natural gas to customers. ANG tanks demand less energy on gas compression, save transport costs for being lighter than CNG and reduce gas flare losses (INGEVITY, 2017).

Several companies have been designing ANG tanks for vehicular use. It is claimed that it can reduces in $50 \%$ the costs of refuelling stations and improve safety due to its controlled release of gas at low pressure (ANGP, 2017).

\subsubsection{Design Approaches}

The major drawback of ANG tanks is that their internal temperature variation prevents them from reaching their full capacity of gas adsorption in fast filling and emptying cycles. Since the adsorption reaction is exothermic, when a molecule of gas is adsorbed by the surface, heat is released. This behaviour is detrimental to the storage of gas by means of adsorption because the saturation of adsorbed gas decreases with the increase of temperature. Conversely, when filling an adsorbent bed, the probability of a molecule being adsorbed is increasingly lower, because of the heat generated by previous adsorption reactions. In turn, the desorption reaction is endothermic, consuming heat when molecules detaches from the surface. This behaviour is also disadvantageous, since it makes the desorption of molecules harder due to the fall in temperature, hindering the gas recovery from the adsorbent.

Therefore, effective ANG tanks can not rely on materials presenting high capacity of adsorption only. It is necessary to ensure appropriate thermal management in order to allow reasonable short filling and emptying cycles. Given the poor conductivity typically presented by adsorbent materials, thermal management is essential to the feasibility of ANG tanks.

Shorter cycles can be achieved by varying the tank shape and boundary conditions. Sahoo et al. (2011) analysed different inlet flow rates, aspect ratios and convection co- 
efficients. Naturally, the achieved storage density has shown to be higher for slim tanks subjected to high heat convection with the surroundings.

Cooling elements can also be added to the tank. Mota et al. (2004) relies on a cooling jacket traversed by hot exhaust gases, imposing forced convection to the tank during a desorption cycle. Subjected to exhaust gases at $80^{\circ} \mathrm{C}$, the jacketed tank has presented a final average temperature $30^{\circ} \mathrm{C}$ hotter than the conventional reference tank in a 3-hour cycle, which results in an important increase of the net deliverable capacity. It is also reported that the inclusion of radially-extruded fins, arbitrary distributed throughout the adsorbed bed, has improved the deliverable capacity in $5 \%$ more. Based on such results, Mota et al. (2004) recommends future optimisation of fins distribution.

The distribution of fins across the adsorbent bed is also recommended by Vasiliev et al. (2014) on the adsorption of hydrogen on Busofit activated carbon. In this case, fins are connected to a double-pipe heat exchanger traversed by a cooling fluid at $233 \mathrm{~K}$, which is built in the centre of the adsorbent bed. Similar to Mota et al. (2004), fins have allowed $25 \%$ more gas to be adsorbed, despite being arbitrarily distributed.

It is important to remark, however, that the models presented by Mota et al. (2004) and Vasiliev et al. (2014) are dependent on intense active cooling, based on cooling fluids at $80^{\circ} \mathrm{C}$ and $-40^{\circ} \mathrm{C}$, respectively, flowing across the whole adsorbent bed length. The gains achievable with such methods are naturally high, although the energy required to run such systems can make them less efficient per unit of gas stored. Therefore, an exergy assessment would be necessary in order to determine the feasibility of such ANG tanks.

When active cooling is inefficient or infeasible, some alternative methods based on passive thermal management are interesting. Toledo et al. (2013) studies the adsorption and desorption of $\mathrm{CO}_{2}$ on systems containing spheres of Phase Change Materials (PCM). These materials behave as heat sinks, due to their high latent heat, and can be distributed across the adsorbent bed in order to control temperature variations.

\subsubsection{Numerical Modelling}

Several numerical methods have been applied to model adsorption systems. Hirata et al. (2009) adopts an hybrid numerical-analytical method based on the Generalised Integral Transform Technique (GITT) for modelling a simple one-dimensional problem considering the energy balance only, which is overly simplified for the scope of this work.

In Thacker et al. (2014) and Mota et al. (1996), the Partial Differential Equations 
(PDE) are reduced to a set of Ordinary Differential Equations (ODE) by interior orthogonal collocation, in which the non-symmetry of the collocation matrix is disadvantageous (SUN et al., 1996).

The Finite Volume Method (FVM) is adopted in Santos et al. (2009), Mota et al. (2004) and Vasiliev et al. (2014) and the Finite Element Method (FEM) in Yonge (1992) and Sahoo et al. (2011). FVM and FEM are the most popular techniques for solving problems with coupled heat transfer and fluid flow, sharing the same fundamental idea of satisfying a weak form, differing on the choice of the weighting function (CHAO et al., 2002). Given the vast literature of latter methods, the FEM method is adopted in this work.

\subsection{Optimisation Methods applied to Fluids and Heat Transfer Problems}

Optimisation methods are suitable to the design of devices subjected to thermofluidic interaction, such as in adsorption systems. A key aspect when designing such systems is the way trial geometries are represented through the design variables. According to Hall et al. (2017), there are five main groups of parametrisation, based on (i) surface points, (ii) control points, (iii) level sets, (iv) descriptive functions or (v) volumetric representation.

Methods in the first group considers the mobility of every node on the surface mesh, such that their coordinates are the design variables of the optimisation problem (REUTHER et al., 1999). This method requires that the gradient of the objective function must be smoothed to avoid noisy surface shapes. It is widely adopted for aerodynamic optimisation, although presenting high computational cost and slow convergence rates. In the second group, control points are defined in order to reduce the problem size. Splines, Bezier curves or B-Splines are some of the possibilities for interpolating smooth surfaces based on fewer points. Free-Form Deformation (FFD) (SEDERBERG; PARRY, 1986) and Radial Basis Function (RBF) (MORRIS et al., 2008) are methods in which control points are used to deform the mesh through a relationship between the position of these points and the mesh nodes. In the third group, surface boundaries are defined by level sets of a function related to the sensitivity of each region to the objective function. The level set function is evolved according to a time step analogy, in which at every step a new geometry is defined at the chosen level (usually zero) (DIJK et al., 2013). In the fourth group, shape functions are associated with class functions, which represent basic types of bodies. In methods belonging to this group, the design variables can scale shape func- 
tions, which will define the body constrained to class functions, or be used as coefficients to multiply perturbation functions (HALL et al., 2017).

In the last group, free boundaries are represented by the concept of fractional Volume of Fluid (VoF), where the fraction of material stored in grid cells defines solid and fluid regions. The Piecewise Linear Interface Calculation (PLIC) method (ANISZEWSKI et al., 2014) uses the fluxes of the fraction function to determine a well-defined solid-fluid interface. Other methods ignore the notion of surfaces by only controlling the extent to which a given point is solid, as is the case of the density approach in topology optimisation. Volume-based methods present the advantage of handling any topology change, allowing the creation and disappearance of surfaces across the domain.

Topology optimisation seeks optimised distributions of different materials inside a fixed design domain in order to maximise or minimise an objective function. Topology optimisation is usually implemented in association with FEM, considering a design variable in each finite element or node in the domain. The design variables are generally called pseudo-densities and range from 0 to 1, in order to represent the presence of material and absence of material (void) or two different materials. It is considered one of the most generic methods of optimisation as it is able to represent any material contour inside the design domain.

In structural problems, 0 and 1 often denotes the absence and presence of material, respectively. In thermal problems, a common optimisation problem is to distribute materials with different conductivities along the design domain, being one material represented by 0 and the other by 1 . The optimisation of fluidic problems is addressed based on the governing equation of porous media flow to represent solid regions. Solid materials are represented by pseudo-densities equal to 0 and are considered to be a porous medium with a very low permeability, while fluid regions are denoted by 1 and governed by Navier-Stokes equations.

The implementation of topology optimisation methods to heat transfer problems was introduced by Xie et al. (1999), which adopt an Evolutionary Structural Optimisation (ESO) approach by removing under-utilised elements from the domain mesh. In the ESO method, elements are removed according to a rejection criterion until reaching a steady state. Such criterion is then iteratively updated following an evolution rate, culminating in the final topology. In Turteltaub (2001), the optimisation of transient heat conduction problems is studied by varying the material properties inside a fixed design domain by following the SIMP scheme and including a regularisation term on the objective function. 
However, the results presented did not cover the solid-void optimisation case, which is later addressed by Sigmund (2001) by designing thermal and electro-thermal micro-actuators subjected to top heat convection only.

In order to take the side convection into account in the design of electro-thermal actuators, the work of Yoon \& Kim (2005) is based on the Element Connectivity Parametrisation (ECP) method, where the design variables are the links between elements instead of their densities. Bruns (2007) gives special attention to convection-dominated problems, by discussing their susceptibility to numerical instabilities inherent to the density dependent interpolation schemes commonly used in topology optimisation. Such instabilities are dealt with by ensuring that the convection term contributions are lumped matrices.

Borrvall \& Petersson (2003) applied topology optimisation to the design of fluid systems seeking to minimise the power dissipation in fluid flow channels. A well-posed optimisation problem is introduced, presenting solution convergence to a binary function space as the mesh is refined. It is based on a material model with a different penalisation scheme, which will be detailed further in Section 3.4.1. Gersborg-Hansen et al. (2005) initially extended this approach to incompressible laminar viscous flow at moderate Reynolds numbers, thus solving a non-linear problem. Then, Guest \& Prevost (2006) proposed a new generalized method, by combining and scaling the Stokes and Darcy equations.

Yoon (2010) coupled heat transfer to the fluid problem, optimising heat dissipating structures with forced convection. This multiphysics optimisation is also approached by Koga et al. (2013) and Matsumori et al. (2013) with similar purposes. Kontoleontos et al. (2012) widened the set of problems tested, including three-dimensional manifolds. Yoon (2012) optimised Electro-Thermo-Mechanical (ETM) actuators by considering their interaction with the surrounding fluid.

Despite the theory of topology optimisation applied to fluid mechanics being based on the variation of porosity, where media presenting very low porosity emulate impermeable solid regions, few works applied the method to the optimisation of actual porous media. The concept of impermeability adopted in Guest \& Prevost (2006) and in Borrvall \& Petersson (2003) considers that a material presenting permeability $K=1.00 \times 10^{-8} \mathrm{~m}^{2}$ behaves like a wall, while in typical ANG tanks the fluid flow permeates an activated carbon presenting $K=3.70 \times 10^{-10} \mathrm{~m}^{2}$ (SAHOO et al., 2011).

On structural design, Andreasen \& Sigmund (2013) presents an implementation of topology optimisation for saturated poroelastic media, with respect to the macroscopic material distribution based on the homogenisation theory. The method is applied to the 
design of shock absorbers, where the energy dissipation is due to the flow resistance.

The use of topology optimisation for the design of adsorbed systems is not yet reported in literature. Figure 1.3 presents the topology optimisation procedure for designing ANG tanks. A fixed initial domain with given boundary conditions is discretised in finite elements. Pseudo-porosities are associated with each element and their values are optimised aiming to maximise or minimise a given objective function, resulting in a final topology. A post-processing step is then necessary, in order to recover the discrete nature of the problem and to smoothen the contours of the material distribution. Finally, the postprocessed topology can be verified numerically through other software before proceeding to manufacturing.

1

INITIAL DOMAIN

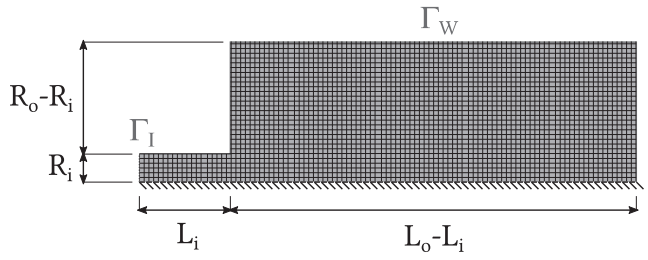

3 POST-PROCESSING

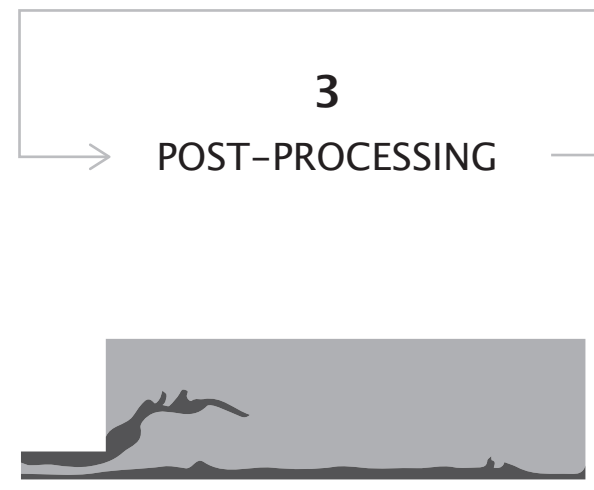

FINAL TOPOLOGY

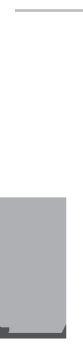

4 VERIFICATION

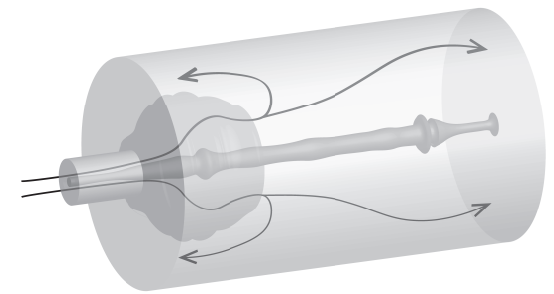

Figure 1.3: Topology optimisation procedure.

\subsection{Motivation}

Adsorption systems play an important role in a wide range of applications, with a vast range of commercial products reliant on the process. Aiming to improve the performance of adsorption devices, past research has been limited to the improvement of adsorbent characteristics, such as the pores geometry and distribution (BILOE et al., 2002). The 
design of adsorption systems by stating and solving an actual optimisation problem has not been reported in literature. Topology optimisation is suitable to address the design of such systems and expand their current applications.

\subsection{Objectives}

This work proposes a method for the design of adsorption systems based on topology optimisation by seeking the following objectives:

- To formulate a comprehensive numerical model of gas adsorption on porous media;

- To formulate topology optimisation problems regarding the maximisation of the mass of gas adsorbed in Adsorbed Natural Gas (ANG) tanks;

- To obtain optimised ANG tank layouts able to adsorb gas more efficiently.

\subsection{Scientific Contributions}

This research addresses some previously unexplored topics, presenting the following scientific contributions:

- Statement of weak and discrete formulations of the adsorption problem and its adjoint system;

- Development of an optimisation method for adsorption systems;

- Definition of a novel material model based on offset hyperbolic tangents;

- Presentation of optimised Adsorbed Natural Gas (ANG) tanks based on the distribution of cooling materials;

\subsection{Thesis Outline}

This document is organized as follows. In Chapter 2, the governing equations of the adsorption phenomenon are presented. In Chapter 3, topology optimisation problems regarding ANG tanks are defined. Chapter 4 details the numerical implementation of the proposed model. In Chapter 5, layouts of ANG tanks obtained by topology optimisation are presented. Chapter 6 reports overall conclusions on the optimisation of adsorption systems. 


\section{Modelling of Adsorption Systems}

The adsorption problem is governed by typical fluidic and thermal equations applied to porous media coupled with equations defining the interactions between adsorbent and adsorbate. This Chapter presents the general assumptions and the governing equations considered when modelling the adsorption problem. The weak formulation of the governing equations is also derived.

\subsection{Modelling Flow in Porous Media}

The modelling of the actual flow in porous media is very challenging, given the complex network of channels presented by such materials. By regarding the porous media as a continuum, it is possible to approximate the macroscopic fluid motion through its channels by taking an average of the microscopic flow within a Representative Elementary Volume (REV) (WHITAKER, 1986). Figure 2.1 shows on the right the continuum interpretation of the porous medium presented on the left. The averaged velocity of the flow inside each $\mathrm{REV}$ is represented by the arrows.

The Darcy's law is an approximation which describes the fluid flow in porous media that is valid for a limited range of low velocities. Deviations presented by experimental results motivated the consideration of microscopic inertial effects, as in the Forchheimer equation (MULJADI et al., 2016), in which

$$
-\nabla p=\frac{\mu_{f}}{K} \mathbf{v}_{g}+\beta \rho_{g}\left|\mathbf{u}_{g}\right|^{2} \mathbf{n},
$$

where $\rho_{g}$ denotes the fluid density, $\beta$ is the non-Darcy coefficient and $\mathbf{n}$ is a unit vector in the direction of $\nabla P$. 


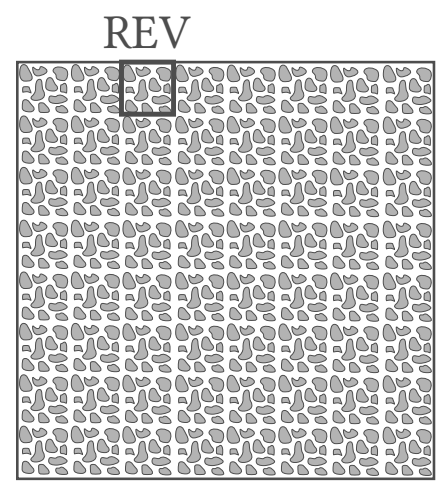

REV
\begin{tabular}{|l|l|l|l|l|l|l|l|}
\hline 1 & 1 & 1 & 1 & 1 & 1 & 1 & 1 \\
\hline 1 & 1 & 1 & 1 & 1 & 1 & 1 & 1 \\
\hline 1 & 1 & 1 & 1 & 1 & 1 & 1 & 1 \\
\hline 1 & 1 & 1 & 1 & 1 & 1 & 1 & 1 \\
\hline 1 & 1 & 1 & 1 & 1 & 1 & 1 & 1 \\
\hline 1 & 1 & 1 & 1 & 1 & 1 & 1 & 1 \\
\hline 1 & 1 & 1 & 1 & 1 & 1 & 1 & 1 \\
\hline 1 & 1 & 1 & 1 & 1 & 1 & 1 & 1 \\
\hline
\end{tabular}

Figure 2.1: Continuum modelling of porous media based on Representative Elementary Volume (REV).

In order to determine the cases when non-Darcy effects are meaningful, the Reynolds number was the first criterion to be used. It is based on the assumption that non-Darcy effects in porous media are similar to turbulence in conduits, in which Reynolds number is given by (ZENG; GRIGG, 2006)

$$
R e=\frac{\rho_{g} \varnothing_{p} \mathbf{v}_{g}}{\mu},
$$

where the conduit diameter is considered to be in the same magnitude of the average particle diameter $\phi_{p}$ in the porous medium.

Previous research present a wide range for the transition region between Darcy and non-Darcy porous media flow. Different authors report discrepant transition regions, with Reynolds number ranging from 0.1 to 1000 (MULJADI et al., 2016). Due to the lack of a precise delimitation of this region, criteria based on Reynolds number are not widely adopted (ZENG; GRIGG, 2006).

A second criterion is based on the Forchheimer number, which is given by (ZENG; GRIGG, 2006)

$$
F_{O}=\frac{\rho_{g} K \beta \mathbf{v}_{g}}{\mu}
$$

where $\beta$ denotes the non-Darcy coefficient.

While the measurement of the particle diameter $\emptyset_{p}$ in the Reynolds number is not trivial, the permeability $K$ and the coefficient $\beta$ involved in the Forchheimer number are easily obtained experimentally by measuring the pressure drop in a specimen for a range of different flow rates (ZENG; GRIGG, 2006). It has been found that a Forchheimer number equal to 0.11 is a good reference for the acceptance of Darcy's law approximation in porous media. The meaning of a flow presenting $F_{O}=0.11$ is that non-Darcy effects represent $10 \%$ of the forces involved. 
In modelling ANG tanks, Mota et al. (2004) and Sahoo et al. (2011) consider all inertial and viscous effects negligible and adopt pure Darcy's law. Mota et al. (1996), Rahman et al. (2011) and Vasiliev et al. (2014) consider additional terms for accounting the inertial effects by following Ergun or Forchheimer equations. In general, models neglecting non-Darcy effects have presented good agreement with experimental validation, being preferred in order to reduce computational cost.

\subsection{Modelling Adsorption Equilibrium and Kinetics}

Adsorption is modelled as the retention of molecules from a solute with concentration $C_{0}$ (relative to the fluid phase volume) on solid particles at a concentration $q$ (relative to the solid phase mass). Such retention reduces the number of molecules on the mobile phase, resulting in a new solute concentration $C$. Given a fixed temperature, a relationship $q=f(C)$ is observed, which is named the sorption isotherm. This process is expressed by (LIMOUSIN et al., 2007)

$$
q=\frac{V}{M}\left(C_{0}-C\right)+q_{0}
$$

where $V$ is fluid phase volume, $M$ is the solid phase mass and $q_{0}$ is the initial concentration of adsorbed molecules on solid particles.

The desorption process is the release of solute molecules from the solid particles, increasing the concentration of the liquid phase. It can be noted that the same equation is valid for modelling both adsorption and desorption processes, given that for adsorption $C_{0}>C$ and $q_{0}<q$ while for desorption $C_{0}<C$ and $q_{0}>q$. Figure 2.2 illustrates these processes.

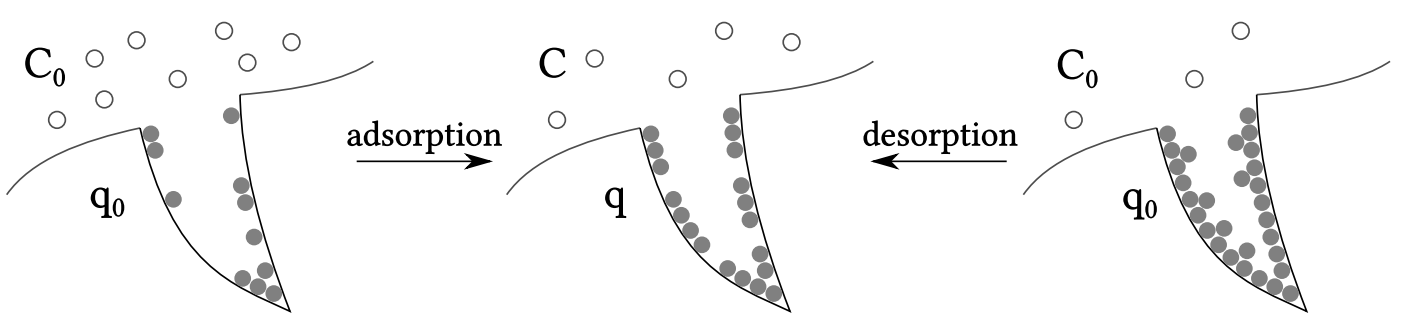

Figure 2.2: Adsorption and desorption processes.

Adsorption process requires an energy of activation, $E_{a}$, and is exothermic, presenting an isosteric heat of adsorption $\Delta H$. In turn, the desorption reaction is endothermic and requires an energy of activation, $E_{d}$. Figure 2.3 the variation of energy in an exothermic reaction along the reaction pathway. 


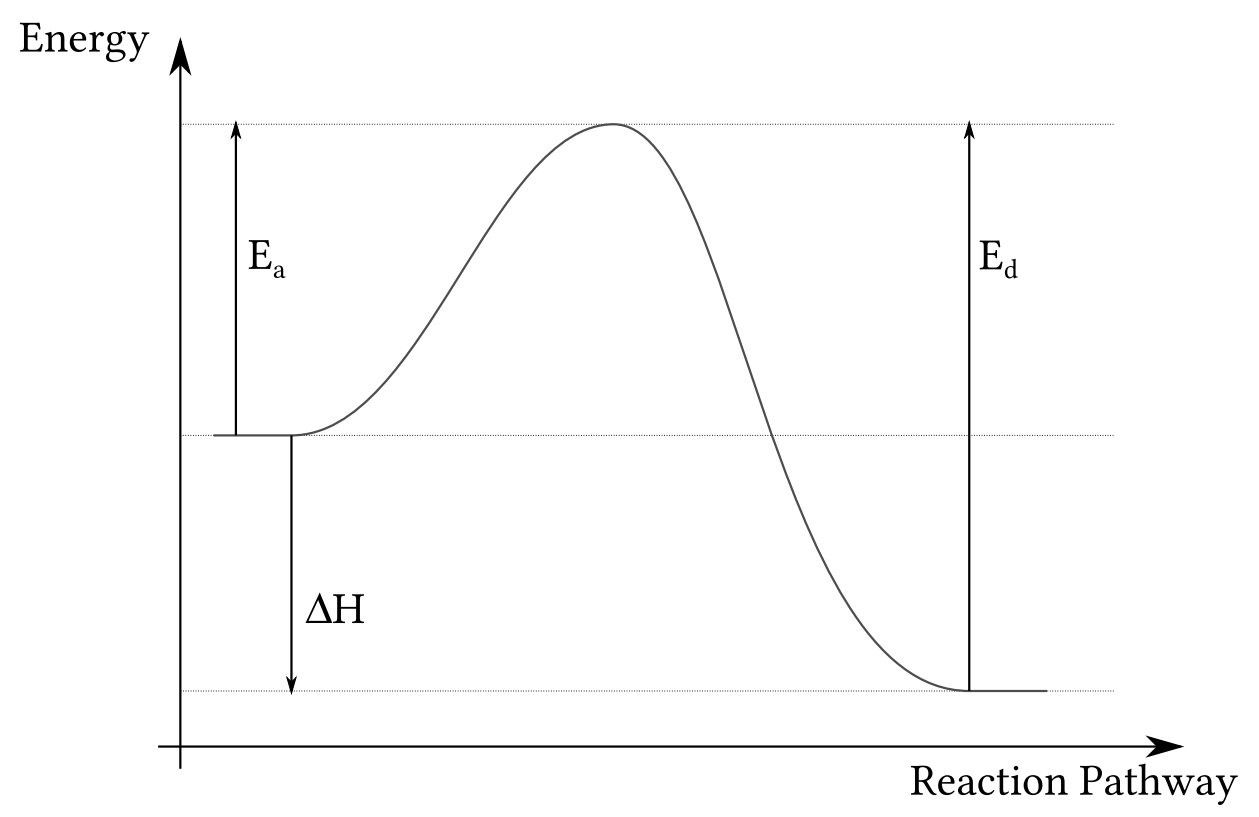

Figure 2.3: Adsorption energetics (MCBRIDE, 1994).

Giles (1974) divides the isotherms into four classes according to their initial slope termed as S, L (Langmuir), $\mathrm{H}$ (high affinity), and $\mathrm{C}$ (constant partition), which are depicted in Figure 2.4. C-isotherm considers a simple linear function for expressing $q=$ $f(C)$, which means that the sorbate has infinite adsorption sites. L-isotherm assumes steady decrease on the concentrations ratio, presenting a well defined plateau in some cases. H-isotherm is suitable when the affinity between adsorbate and adsorbent is very high and the available sites are occupied promptly. S-isotherm presents an inflection point which is the result of the opposition of two mechanisms: the low rate observed for low concentrations reproduces the difficulty that some adsorbates have to attach to the adsorbent and after the adsorption of the first molecules the adsorption of others is favoured through a phenomenon named cooperative adsorption.

L-isotherm and H-isotherm are also know as concave isotherms and several different models are based on them, such as Freundlich, Langmuir, Dubinin, Polanyi, Kisarov and Vacancy Solution Model (WOOD, 2002).

According to the Freundlich models, a plateau is never reached, even for very high concentrations. It is given by (LIMOUSIN et al., 2007)

$$
q=F C^{n}
$$

where $F$ and $n$ are constants obtained experimentally, being $n<1$.

Langmuir models are based on the assumption that the adsorbate presents a limited capacity of adsorption $q_{\max }$. It is derived from the kinetics of adsorption and desorption. 


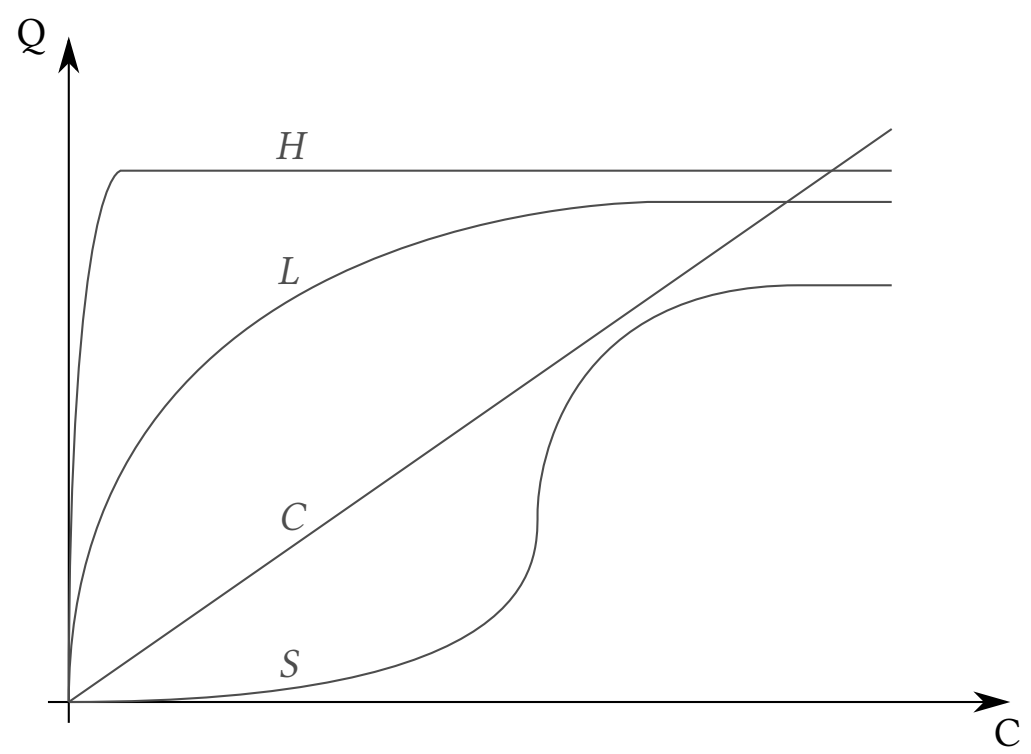

Figure 2.4: Classes of isotherms (GILES, 1974).

Given that the rate of adsorption is proportional to the vacant sites for adsorption, it can be expressed as (PATIHA et al., 2016)

$$
\frac{\partial q}{\partial t}=k_{a}\left(q_{\max }-q\right) C
$$

where $k_{a}$ is the kinetic coefficient of adsorption obtained experimentally for each adsorbentadsorbate pair.

The desorption rate is proportional to the occupied sites (PATIHA et al., 2016), which is expressed as

$$
\frac{\partial q}{\partial t}=k_{d} q
$$

where $k_{d}$ is the kinetic coefficient of desorption.

At equilibrium, the rates of adsorption and desorption are equal and $q$ is given by (PATIHA et al., 2016)

$$
q=\frac{q_{\max } C}{\frac{k_{d}}{k_{a}}+C} .
$$

The Dubinin models are the most versatile methods of expressing adsorption equilibrium, being expressed by means of adsorbate and adsorbent properties and temperature (WOOD, 2002). It relies on the Polanyi's adsorption potential theory, which defines a potential equal to the change in free energy from the bulk liquid to the adsorbed state, taking the vapour saturation pressure $p_{\text {sat }}$ as reference (WOOD, 2001). It is expressed as

$$
A=R T\left(\frac{P_{s a t}}{P}\right)
$$


The Dubinin isotherm is then defined in terms of volume of micro-pores $W$ occupied by adsorbate (WOOD, 2001), given by

$$
W=W_{0} \exp \left(-\left(\frac{A}{\beta E_{0}}\right)^{n}\right)
$$

where $W_{0}$ is the total volume of micro-pores and the exponent $n$ can assume different values depending on the specific model, being equal to 2 for Dubinin-Radushkevich isotherms. $E_{0}$ and $\beta$ denote, respectively, the characteristic adsorption energy for the reference vapour and the affinity of the adsorbate to the adsorbent, usually taking benzene as reference $\left(\beta_{\text {benzene }}=1\right)$.

The kinetics of adsorption consists of a sequence of three steps: (i) mass transfer of the adsorbate from the solution to the external surface of the adsorbent, (ii) diffusion through the adsorbent and (iii) adsorption itself (LARGITTE; PASQUIER, 2016). When modelling adsorption kinetics, it is usual to assume that either the diffusion or the actual adsorption is the rate limiting and dictates the rate of the process as a whole.

Two main models are reported for adsorption kinetics taking the intra-particle diffusion as the limiting step: Crank and Weber-Morris. Crank model considers homogeneous diffusion across an adsorbate consisting of spheres, which is defined as (LARGITTE; PASQUIER, 2016)

$$
\frac{\partial q}{\partial t}=\frac{D_{S}}{r^{2}} \frac{\partial}{\partial r}\left(r^{2} \frac{q}{r}\right)
$$

where $D_{S}$ is the surface diffusivity and $r$ denotes the radial distance.

Weber-Morris model is based on the empirical observation that the uptake varies nearly proportionally with $\sqrt{t}$ (ALKAN et al., 2007). It is expressed by (QIU et al., 2009)

$$
q=k_{\text {intra }} \sqrt{t}
$$

where $k_{i n t r a}$ is the intra-particle diffusion rate constant.

When the adsorption itself is the rate limiting step, Elovich or Lagergren equations are often used to model the phenomenon. Elovich models are expressed by (LARGITTE; PASQUIER, 2016)

$$
\frac{\partial q}{\partial t}=a \exp (-b q)
$$

where $a$ is the initial rate of adsorption and $b$ is a constant obtained experimentally related to the variation of the energy of adsorption.

Lagergren models present an asymptotic approach to the equilibrium of the adsorbed 
phase $q_{e q}$ through a pseudo first order equation given by (LARGITTE; PASQUIER, 2016)

$$
\frac{\partial q}{\partial t}=k_{a}\left(q_{e q}-q\right)
$$

where $k_{a}$ is the adsorption rate constant.

Some other models are derived from the original Lagergren model. One variation consists of the extension of the pseudo first-order form to the pseudo $n$-order laws given by (LARGITTE; PASQUIER, 2016)

$$
\frac{\partial q}{\partial t}=k_{a}\left(q_{e q}-q\right)^{n}
$$

where $n$ defines the pseudo order of the equation.

Another Lagergren derived model, know as Langmuir, includes the consideration of the reversibility of the adsorption reaction by adding the desorption kinetics as (LARGITTE; PASQUIER, 2016)

$$
\frac{\partial q}{\partial t}=k_{a}\left(q_{e q}-q\right)-k_{d} q
$$

where $k_{d}$ is the desorption rate constant.

In ANG tanks, the equation of Dubinin-Astakhov (DA), or its variation DubininRadushevich (DR), is adopted by most authors for predict the amount of gas adsorbed at the equilibrium and the kinetics of adsorption is approximated by the Lagergren model (RAHMAN et al., 2011; SAHOO et al., 2011; VASILIEV et al., 2014). Alternatively, Mota et al. (1996) adopts a Langmuir-type prediction for the equilibrium and suggests that the kinetics of adsorption can be neglected for the typical intraparticle diffusional time constants of adsorbent beds. This assumption is generally valid, but also depends on other factors, such as the energy of activation, and it will be studied further in this work.

\subsection{Governing Equations}

A typical sorption domain $\Omega$ is illustrated in Figure 2.5, where $\Gamma_{W}$ and $\Gamma_{L}$ denote subregions of $\partial \Omega$ where boundary conditions of wall and inlet/outlet are imposed, respectively.

In this work, the following assumptions are made:

Ideal gases: in the systems considered, gases are considered to present ideal thermo- 


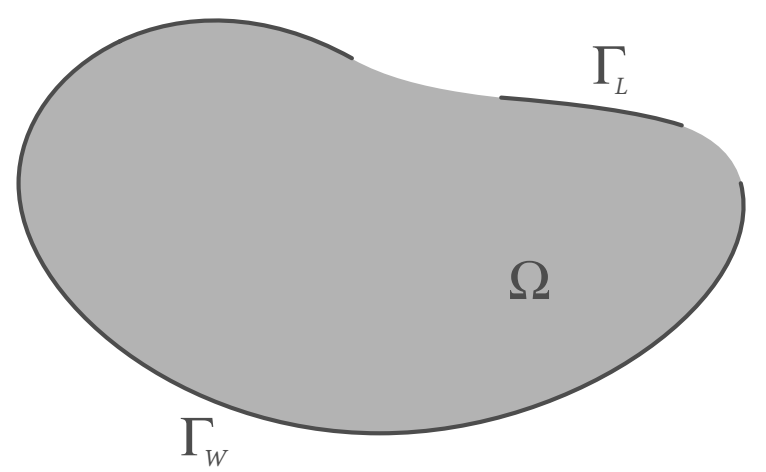

Figure 2.5: Adsorption domain.

dynamic behaviour (SAHOO et al., 2011), therefore, their densities are described by

$$
\rho_{g}=\frac{M_{g} P}{R_{g} T}
$$

where $M_{g}$ is the molecular gas mass, $R_{g}$ is the universal gas constant, $P$ is the gas pressure and $T$ is the gas temperature.

Darcy's Porous Media: non-Darcy effects are neglected in the fluid flow, according to Eq. (2.1) with $\beta=0$.

Local thermal equilibrium: the sorbent bed is locally in thermal equilibrium with the gas (MOTA et al., 2004). All intra-particle and film resistances in heat and mass transfer are neglected.

Negligible viscous forces: the viscous forces are neglected due to the low gas viscosity and the relatively large bed-particle diameter ratio (MOTA et al., 2004).

Constant specific heat capacity: the specific heat capacity is considered to be constant within the typical range of temperatures observed in sorption systems. Its variation is neglected since it usually varies around only 8\% (MOTA et al., 2004) in a range of $100 \mathrm{~K}$.

Constant adsorption enthalpy: this work considers an approximation in which $\Delta H$ assumes a constant mean value within the range of pressures and temperatures studied (SAHOO et al., 2011).

Lagergren adsorption model: For the studied cases, it is considered that the film diffusion is dominant and the rate of sorption is governed by Lagergren model (VASILIEV et al., 2014). 


\subsubsection{Natural Material Models}

Effective properties of porous media are determined by material models based on macro-porosity $\left(\varepsilon_{M}\right)$ and micro-porosity $\left(\varepsilon_{m}\right)$. Given these two classes of porosity, the total porosity $\left(\varepsilon_{t}\right)$ is defined as

$$
\varepsilon_{t}=\varepsilon_{M}+\left(1-\varepsilon_{M}\right) \varepsilon_{m}
$$

Density: the density of the system is given by the sum of gaseous and solid phase weighted by the total porosity, $\varepsilon_{t}$, with the addition of the density of the adsorbed phase, based on its concentration, $q$, as

$$
\rho_{f}\left(\varepsilon_{t}\right)=\varepsilon_{t} \rho_{g}+\left(1-\varepsilon_{t}\right) \rho_{s}+\left(1-\varepsilon_{t}\right) \rho_{s} q
$$

Permeability: dependent on the macro-porosity, following the Kozeny-Carman's model for spherical particles (CARMAN, 1937), given by

$$
K_{s}\left(\varepsilon_{M}\right)=\frac{\emptyset_{s}^{2} \varepsilon_{M}^{3}}{72 \tau_{s}\left(1-\varepsilon_{M}\right)^{2}},
$$

where $\emptyset_{s}$ is the effective characteristic particle diameter and $\tau_{s}$ denotes the pores tortuosity.

Thermal Conductivity: it can be given by both Maxwell-Eucken's or Russel's relations (SMITH et al., 2013). The former considers the effect of large spherical pores with thin walls and the latter the effect of a cubic pore inclusion in the material matrix by considering a parallel/series resistor. Here, the conductivity of the thin film of gas adsorbed, $k_{q}$, is neglected and the Maxwell-Eucken relation is adopted to interpolate the gaseous and solid conductivities (SMITH et al., 2013), given by

$$
k_{f}\left(\varepsilon_{t}\right)=k_{s} \frac{k_{g}+2 k_{s}+2 \varepsilon_{t}\left(k_{g}-k_{s}\right)}{k_{g}+2 k_{s}-\varepsilon_{t}\left(k_{g}-k_{s}\right)}
$$

where $k_{s}$ and $k_{g}$ are the thermal conductivities for the solid and gas, respectively.

\subsubsection{Adsorption and Desorption Kinetics}

The concentration $q$ represents the mass of adsorbed phase per mass of adsorbent. Therefore, the density of adsorbed phase in reference to the volume of the adsorbent bed, $Q$, is given by

$$
Q=\left(1-\varepsilon_{t}\right) \rho_{s} q
$$


The specific volume of micro-pores, $w_{s}$, represents the volume of micro-pores per mass of adsorbent. The ratio of micro-pores volume per adsorbent bed volume, $W_{s}$, is then given by

$$
W_{s}=\left(1-\varepsilon_{t}\right) \rho_{s} w_{s}
$$

From the equilibrium concentration of adsorbed gas, $q_{e q}$, (SAHOO et al., 2011), the equilibrium density of adsorbed gas is given by

$$
Q_{e q}=\rho_{a d g} W_{s} \exp \left(-\left(\frac{A}{\beta E_{0}}\right)^{n_{f}}\right),
$$

where $\beta$ is the affinity coefficient of the adsorbent-adsorbate pair, $E_{0}$ is the characteristic energy of adsorption and $n_{s}$ stands for the effective micro-pores dispersion.

The local adsorbed gas density, $\rho_{a d g}$, is given by (SAHOO et al., 2011)

$$
\rho_{a d g}=\frac{\rho_{\text {boil }}}{\exp \left(\alpha_{e}\left(T-T_{b o i l}\right)\right)}
$$

where $\rho_{\text {boil }}$ is the gas density at boiling point temperature $\left(T_{b o i l}\right)$ and $\alpha_{e}$ is the mean value of the thermal expansion of liquefied gases.

Polanyi's adsorption potential, $A$, is given by

$$
A=R_{g} T \ln \left(\frac{P_{\text {sat }}}{P}\right),
$$

which saturated pressure $P_{\text {sat }}$ is given by

$$
P_{s a t}=P_{c r}\left(\frac{T}{T_{c r}}\right)^{2},
$$

where $P_{c r}$ and $T_{c r}$ are, respectively, the critical pressure and temperature of the gas.

The rate of adsorption decreases as more gas is adsorbed, following the Linear Driving Force (LDF) model given by(MOTA et al., 1996)

$$
\begin{aligned}
& \frac{\partial Q}{\partial t}=G\left(Q_{e q}-Q\right) \text { in } \Omega \times\left[0, t_{f}\right] \\
& Q=Q_{\text {ini }} \text { in } \Omega \text { at } t=0,
\end{aligned}
$$

where the coefficient $G$, which dictates the adsorption and desorption kinetics, is given by (VASILIEV et al., 2011)

$$
G=D \exp \left(\frac{-E_{[a, d]}}{R_{g} T}\right)
$$

where the activation energy of adsorption $\left(E_{a}\right)$ is considered when $Q \leq Q_{e q}$ and the activation energy of desorption $\left(E_{d}\right)$ otherwise. $D$ is the intra-particle diffusional time 
constant, given by (SAHOO et al., 2011)

$$
D=4 \frac{D_{0}}{\phi_{s}^{2}}
$$

where $\emptyset_{s}$ is the characteristic particle diameter and $D_{0}$ is the gas diffusivity.

\subsubsection{Continuity}

In adsorption systems, the coupled continuity equation of gaseous, solid and adsorbed phases is expressed by

$$
\frac{\partial}{\partial t}\left(\varepsilon_{t} \rho_{g}+\left(1-\varepsilon_{t}\right) \rho_{s}+Q\right)+\nabla \cdot\left(\rho_{g} \mathbf{v}_{g}\right)=0 \text { in } \Omega \times\left[0, t_{f}\right],
$$

where $\rho_{s}$ is the bulk adsorbent density, which is constant.

The continuity equation considering ideal gases and Darcy's laws becomes (SAHOO et al., 2011)

$$
\frac{\varepsilon_{t} M_{g}}{R_{g}} \frac{\partial}{\partial t}\left(\frac{P}{T}\right)+\frac{\partial Q}{\partial t}-\frac{M_{g}}{R_{g} \mu_{g}} \nabla \cdot\left(K_{s} \frac{P}{T} \nabla P\right)=0 \quad \text { in } \Omega \times\left[0, t_{f}\right],
$$

where the solid density $\rho_{s}$ is considered constant.

By expanding the transient derivative term, continuity is given by

$$
\begin{aligned}
\frac{\varepsilon_{t} M_{g}}{R_{g}}\left(\frac{1}{T} \frac{\partial P}{\partial t}-\frac{P}{T^{2}} \frac{\partial T}{\partial t}\right)+\frac{\partial Q}{\partial t}-\frac{M_{g}}{R_{g} \mu_{g}} \nabla \cdot\left(K_{s} \frac{P}{T} \nabla P\right)=0 & \text { in } \Omega \times\left[0, t_{f}\right], \\
P=P_{\text {ini }} & \text { in } \Omega \text { at } t=0, \\
T=T_{\text {ini }} & \text { in } \Omega \text { at } t=0, \\
Q=Q_{\text {ini }} & \text { in } \Omega \text { at } t=0 .
\end{aligned}
$$

For determining a fixed mass inflow or outflow, a Robin boundary condition is applied on $\Gamma_{L}$ as

$$
-\frac{M_{g} K_{s}}{R_{g} \mu_{g}} \frac{P}{T} \frac{\partial P}{\partial \mathbf{n}}=\rho_{g} \mathbf{v}_{g} \cdot \mathbf{n}=\dot{m}_{l e t} \quad \text { on } \Gamma_{L} \times\left[0, t_{f}\right],
$$

where the mass flow is denoted by $\dot{m}_{\text {let }}$

Despite the imposition of a fixed mass inlet making sense for adsorption, the depletion of a fixed amount of gas during desorption cycles is not possible as the tank is emptied. Therefore, pressure inlet can be set, which represents the connection of the tank to a infinity reservoir at fixed pressure for adsorption or the opening of the tank outlet to the atmosphere for desorption. In order to model the gradual pressure transition inside the tank, a possible approach is to smoothly vary the pressure on $\Gamma_{L}$ according to a sinusoidal 
profile given by

$$
P_{\Gamma_{L}}(t)= \begin{cases}P_{i n i}+\sin ^{2}\left(\frac{\pi}{2} \frac{t}{\sigma t_{f}}\right)\left(P_{l e t}-P_{i n i}\right) & \text { on } \Gamma_{L} \times\left[0, \sigma t_{f}\right] \\ P_{l e t} & \text { on } \Gamma_{L} \times\left[\sigma t_{f}, t_{f}\right],\end{cases}
$$

where $P_{i n i}$ and $P_{l e t}$ denote initial and filling/emptying pressures, $t_{f}$ is the final time and $0<\sigma \leq 1.0$ is a smoothness factor. Figure 2.6 illustrates $P_{\Gamma_{L}}$ curves for adsorption and desorption cycles.

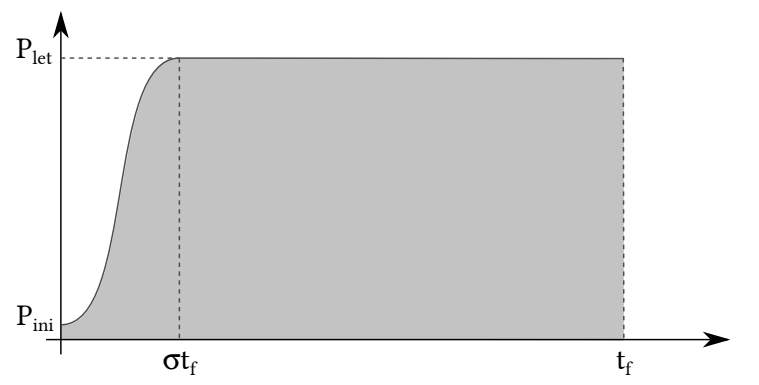

(a)

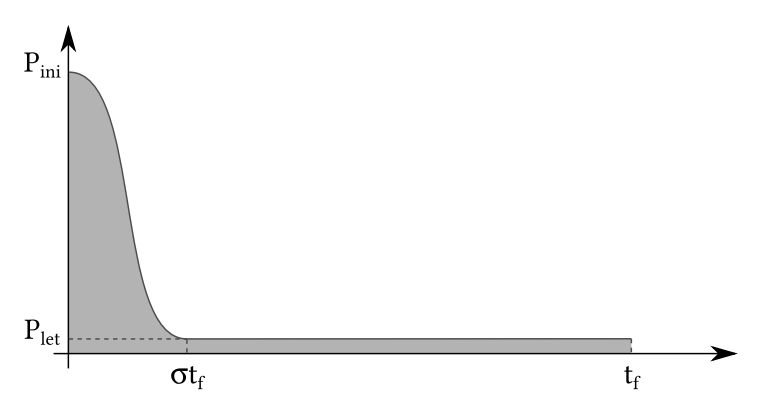

(b)

Figure 2.6: Inlet pressure $\left(P_{\Gamma_{L}}\right)$ during (a) adsorption and (b) desorption cycles.

\subsubsection{Energy Balance}

The energy balance of the gaseous phase is given in terms of internal energy, $u_{g}$, by (BIRD et al., 2007)

$$
\frac{\partial \rho_{g} u_{g}}{\partial t}+\nabla \cdot\left(\rho_{g} u_{g} \mathbf{v}_{g}\right)-\nabla \cdot\left(k_{g} \nabla T\right)+P \nabla \cdot \mathbf{v}_{g}+\boldsymbol{\tau}_{g}: \nabla \mathbf{v}_{g}=0
$$

where $\boldsymbol{\tau}_{g}$ is the viscous stress tensor.

Such balance is rewritten in terms of $C p_{g}$ and $T$ as (BIRD et al., 2007)

$$
\frac{\partial \rho_{g} C p_{g} T}{\partial t}+\nabla \cdot\left(\rho_{g} C p_{g} T \mathbf{v}_{g}\right)-\nabla \cdot\left(k_{g} \nabla T\right)+\boldsymbol{\tau}_{g}: \nabla \mathbf{v}_{g}-\left.\frac{\partial \ln V}{\partial \ln T}\right|_{P} \frac{D P}{D t}-\rho_{g} T \frac{D C p_{g}}{D t}=0
$$

where $D$ denotes the Lagrangian derivative, $C p_{g}$ is considered constant and $\frac{\partial \ln V}{\partial \ln T}=1$ for ideal gases.

Following the assumption that viscous forces are negligible, the energy balance reduces to

$$
\frac{\partial \rho_{g} C p_{g} T}{\partial t}+\nabla \cdot\left(\rho_{g} C p_{g} T \mathbf{v}_{g}\right)-\nabla \cdot\left(k_{g} \nabla T\right)-\frac{\partial P}{\partial t}-\mathbf{v}_{g} \cdot \nabla P=0
$$


which is rearranged as

$$
\begin{array}{r}
\rho_{g} C p_{g} \frac{\partial T}{\partial t}+C p_{g} T\left\{\frac{\partial \rho_{g}}{\partial t}+\nabla \cdot\left(\rho_{g} \mathbf{v}_{g}\right)\right\}_{\mathcal{C}}+\rho_{g} C p_{g} \mathbf{v}_{g} \cdot \nabla T \\
-\nabla \cdot\left(k_{g} \nabla T\right)-\frac{\partial P}{\partial t}-\mathbf{v}_{g} \cdot \nabla P=0
\end{array}
$$

where the continuity equation is observed in $\mathcal{C}$, making these terms to vanish.

By substituting the momentum equation based on the Darcy's law, the balance becomes

$$
\begin{array}{r}
\rho_{g} C p_{g} \frac{\partial T}{\partial t}-\rho_{g} C p_{g} \frac{K_{s}}{\mu_{g}} \nabla P \cdot \nabla T \\
-\nabla \cdot\left(k_{g} \nabla T\right)-\frac{\partial P}{\partial t}+\left\{\frac{K_{s}}{\mu_{g}} \nabla P \cdot \nabla P\right\}_{\approx 0}=0
\end{array}
$$

where the last term is neglected, given the very small pressure gradients observed in the range of permeabilities of interest in this work.

Analogously, the energy balance of the solid phase is given by

$$
\rho_{s} C p_{s} \frac{\partial T}{\partial t}-\nabla \cdot\left(k_{s} \nabla T\right)=0 .
$$

The energy balance associated to the adsorbed phase includes a source term due to the exothermic adsorption reaction, being expressed as

$$
C p_{q} Q \frac{\partial T}{\partial t}-\nabla \cdot\left(k_{q} \nabla T\right)=|\Delta H| \frac{\partial Q}{\partial t}
$$

where $\Delta H$ is the isosteric heat of adsorption.

The energy balance for coupled gaseous, solid and adsorbed phases assuming local thermal equilibrium between phases and ideal gas is then given by (MOTA et al., 2004)

$$
\begin{aligned}
\varepsilon_{t}\left(\frac{C p_{g} M_{g}}{R_{g}} \frac{P}{T} \frac{\partial T}{\partial t}-\frac{\partial P}{\partial t}\right)+\left(1-\varepsilon_{t}\right)\left(\rho_{s} C p_{s} \frac{\partial T}{\partial t}\right)+C p_{g} Q \frac{\partial T}{\partial t} \quad & \text { in } \Omega \times\left[0, t_{f}\right], \\
-\frac{C p_{g} M_{g}}{R_{g} \mu_{g}} K_{s} \frac{P}{T} \nabla P \cdot \nabla T-\nabla \cdot\left(k_{f} \nabla T\right)=|\Delta H| \frac{\partial Q}{\partial t} & \\
P=P_{\text {ini }} & \text { in } \Omega \text { at } t=0, \\
T=T_{\text {ini }} & \text { in } \Omega \text { at } t=0, \\
Q=Q_{\text {ini }} & \text { in } \Omega \text { at } t=0,
\end{aligned}
$$

where the effective thermal conductivity, $k_{f}$, is given by Eq. (2.21).

Due to the local thermal equilibrium assumption, the temperature on $\Gamma_{L}$ can be considered equal to the inlet gas temperature, which is imposed by a Dirichlet boundary 
condition defined as

$$
T=T_{l e t} \quad \text { on } \Gamma_{L} \times\left[0, t_{f}\right]
$$

Alternatively, an inlet heat flux can be considered on $\Gamma_{L}$, which is expressed by a Robin boundary condition (SAHOO et al., 2011) given by

$$
-k_{f} \frac{\partial T}{\partial \mathbf{n}}=-\rho_{g} C p_{g} \mathbf{v}_{g} \cdot \mathbf{n}\left(T-T_{l e t}\right)=\frac{M_{g} C p_{g} K_{s}}{R_{g} \mu_{g}} \frac{P}{T} \nabla P \cdot \mathbf{n}\left(T-T_{l e t}\right) \quad \text { on } \Gamma_{L} \times\left[0, t_{f}\right] .
$$

Assuming that heat convection takes place on the walls, a Robin boundary condition is also defined on $\Gamma_{W}$. Such condition is imposed by

$$
-k_{f} \frac{\partial T}{\partial \mathbf{n}}=h_{W}\left(T-T_{\infty}\right) \quad \text { on } \Gamma_{W} \times\left[0, t_{f}\right] .
$$

On the region $\Gamma \backslash\left(\Gamma_{W} \cup \Gamma_{L}\right)$, since no boundary terms are explicitly defined, the heat flux is null, which represents adiabatic condition.

\subsection{Weak Formulation of Adsorption Systems}

Weak formulations are used to obtain solutions for a set of PDEs by satisfying the integral of the solution on the domain for a family of test functions. Consider the domain $\Omega \subset \mathbb{R}^{n}$, illustrated in Figure 2.5 for $n=2$. The weak formulation of $\mathbb{L}(w)$ is given by its actuation on the test function $v$ as (KARNIADAKIS et al., 2005)

$$
\langle\mathbb{L}(w), v\rangle=\int_{\Omega} \mathbb{L}(w) v d \mathbf{x} \quad \forall v \in H^{1}(\Omega),
$$

where $H^{1}$ denotes the Hilbert space, whose functions have their first derivatives belonging to the Lebesgue space $L^{2}$.

For axisymmetric domains, it is multiplied by $2 \pi r$ and given by

$$
\langle\mathbb{L}(w), v\rangle=2 \pi \int_{\Omega} r \mathbb{L}(w) v d \mathbf{x} \quad \forall v \in H^{1}(\Omega)
$$

where $r$ is the radial coordinate.

On $\Gamma_{N} \subset \partial \Omega$, where Robin boundary condition applies, the weak term is given by (KARNIADAKIS et al., 2005)

$$
\langle\mathbb{L}(w), v\rangle_{\Gamma_{N}}=\oint_{\Gamma_{N}} \mathbb{L}(w) \cdot \mathbf{n} v d s \quad \forall v \in H^{1}(\Omega)
$$


where $\mathbf{n}$ denotes an outward unit vector normal to $\Omega$.

Analogously, for axisymmetric domains, it is given by

$$
\langle\mathbb{L}(w), v\rangle_{\Gamma_{N}}=2 \pi \oint_{\Gamma_{N}} r \mathbb{L}(w) \cdot \mathbf{n} v d s \quad \forall v \in H^{1}(\Omega) .
$$

Considering the Dirichlet boundary condition given by

$$
w=w_{D} \text { on } \Gamma_{D},
$$

the unknown $w$ belongs to the space $\mathcal{W}$ defined as

$$
\mathcal{W}=\left\{w \in H^{1}(\Omega): w=w_{D} \text { on } \Gamma_{D}\right\}
$$

in which $w_{D} \in H^{1 / 2}(\Omega)$ (SA et al., 2016).

Thus, from Eqs. (2.33), (2.43) and (2.28) and integrating by parts the terms involving a divergent operator, the weak forms for continuity, energy balance and adsorption kinetics are given by

$$
\begin{gathered}
\mathbb{M}(P, T, Q)=\left\langle\frac{\varepsilon_{t} M_{g}}{R_{g}} \frac{1}{T} \frac{\partial P}{\partial t}, v_{M}\right\rangle-\left\langle\frac{\varepsilon_{t} M_{g}}{R_{g}} \frac{P}{T^{2}} \frac{\partial T}{\partial t}, v_{M}\right\rangle+\left\langle\frac{\partial Q}{\partial t}, v_{M}\right\rangle \\
+\left\langle\frac{M_{g} K_{s}}{R_{g} \mu_{g}} \frac{P}{T} \nabla P, \nabla v_{M}\right\rangle-\left\langle\frac{M_{g} K_{s}}{R_{g} \mu_{g}} \frac{P}{T} \nabla P, v_{M}\right\rangle_{\Gamma_{N}}=0 \\
\forall v_{M} \in H^{1}(\Omega), \\
\mathbb{E}(P, T, Q)=\left\langle\frac{\varepsilon_{t} C p_{g} M_{g}}{R_{g}} \frac{P}{T} \frac{\partial T}{\partial t}, v_{E}\right\rangle+\left\langle C p_{g} Q \frac{\partial T}{\partial t}, v_{E}\right\rangle \\
+\left\langle\left(1-\varepsilon_{t}\right) \rho_{s} C p_{s} \frac{\partial T}{\partial t}, v_{E}\right\rangle-\left\langle\varepsilon_{t} \frac{\partial P}{\partial t}, v_{E}\right\rangle \\
+\left\langle\frac{\left.C p_{g} M_{g} K_{s} P \frac{P}{T} \nabla P \cdot \nabla T, \nabla v_{E}\right\rangle+\left\langle k_{f} \nabla T, \nabla v_{E}\right\rangle}{R_{g} \mu_{g}}-\left\langle|\Delta H| \frac{\partial Q}{\partial t}, v_{E}\right\rangle\right. \\
-\left\langle\frac{C p_{g} M_{g} K_{s}}{R_{g} \mu_{g}} \frac{P}{T} \nabla P \cdot \nabla T, v_{E}\right\rangle_{\Gamma_{N}}-\left\langle k_{f} \nabla T, v_{E}\right\rangle=0, \\
\forall \Gamma_{N}=0, \forall v_{E} \in H^{1}(\Omega), \\
\mathbb{D}(P, T, Q)=\left\langle\frac{\partial Q}{\partial t}, v_{D}\right\rangle+\left\langle G Q, v_{D}\right\rangle-\left\langle G Q_{e q}, v_{D}\right\rangle=0, \forall v_{D} \in H^{1}(\Omega) .
\end{gathered}
$$


Considering convective heat transfer on the walls,

$$
\left\langle k_{f} \nabla T, v_{E}\right\rangle_{\Gamma_{N}}=-\left\langle h_{W}\left(T-T_{\infty}\right), v_{E}\right\rangle_{\Gamma_{W}} .
$$

Considering mass flow imposition,

$$
\left\langle\frac{M_{g} K_{s}}{R_{g} \mu_{g}} \frac{P}{T} \nabla P, v_{M}\right\rangle_{\Gamma_{N}}=\left\langle\dot{m}_{l e t}, v_{M}\right\rangle_{\Gamma_{L}} .
$$

In the case that no Dirichlet condition is imposed on temperature field, another term remains from the integration by parts on the energy balance, accounting for the heat flux observed on $\Gamma_{L}$ due to the inlet/outlet of gases, given by

$$
\left\langle k_{f} \nabla T, v_{E}\right\rangle_{\Gamma_{N}}=\left\langle C p_{g} T \dot{m}_{l e t}, v_{E}\right\rangle_{\Gamma_{L}} .
$$

Given that $v_{M}, v_{E}$ and $v_{D}$ are arbitrary test functions, Eqs. (2.53)-(2.55) are coupled and the problem reduces to

$$
\mathbb{M}(P, T, Q)+\mathbb{E}(P, T, Q)+\mathbb{D}(P, T, Q)=0
$$

The approximated solution of such weak problem is obtained via FEM as presented in Chapter 4, where a discrete system is derived based on shape functions interpolation. Before addressing this and other numerical matters, the next Chapter is focused on the definition of the theoretical concepts of the optimisation problems studied in this work. 


\section{Optimisation of ANG Tanks}

This Chapter is focused on the design of adsorption systems via topology optimisation. The optimisation concept is discussed, followed by the definition of the objective function for the optimisation of ANG tanks. Finally, traditional material models are reviewed and a novel Offset Hyperbolic Tangents (OHT) model is proposed.

\subsection{Design Concept}

An ANG tank is considered empty when the equilibrium mass of adsorbed gas for atmospheric pressure and temperature $\left(P_{a t m}\right.$ and $\left.T_{a t m}\right)$ is achieved. Similarly, it is considered full when reaching the adsorption equilibrium for the inlet pressure $\left(P_{l e t}\right)$ and atmospheric temperature. Those quantities are expressed by

$$
\begin{aligned}
Q_{e m p t y} & =\int_{\Omega_{a d s}} Q_{e q}\left(P_{a t m}, T_{a t m}\right) d \mathbf{x}, \\
Q_{\text {full }} & =\int_{\Omega_{a d s}} Q_{e q}\left(P_{l e t}, T_{a t m}\right) d \mathbf{x},
\end{aligned}
$$

where $\Omega_{a d s}$ is the adsorbent domain inside the tank.

At the end of an adsorption or desorption cycle, the actual mass of gas adsorbed inside the tank is given by

$$
Q_{f}=\int_{\Omega_{a d s}} Q_{e q}\left(P_{f}, T_{f}\right) d \mathbf{x},
$$

which is well approximated by

$$
Q_{f} \approx \int_{\Omega_{a d s}} Q_{e q}\left(\overline{P_{f}}, \overline{T_{f}}\right) d \mathbf{x},
$$

where $\overline{P_{f}}$ and $\overline{T_{f}}$ denote the average pressure and temperature within the domain $\Omega_{a d s}$ at the end of the cycle. 
The goal when designing ANG tanks, is to make the temperature $\overline{T_{f}}$ as near as possible to the atmospheric temperature $T_{a t m}$ at the end of both adsorption and desorption cycles. This is often very time demanding due to the adsorbent poor conductivity mentioned above. A possible approach to shorten the cycle length is not to occupy the full volume of the tank with adsorbent, replacing it per some high conductive material in certain regions, as demonstrated in the tank depicted in Figure 3.1. The design trade-off is to not remove too much adsorbent when introducing non-adsorbent material to make the temperature $\overline{T_{f}}$ nearer to the atmospheric value.

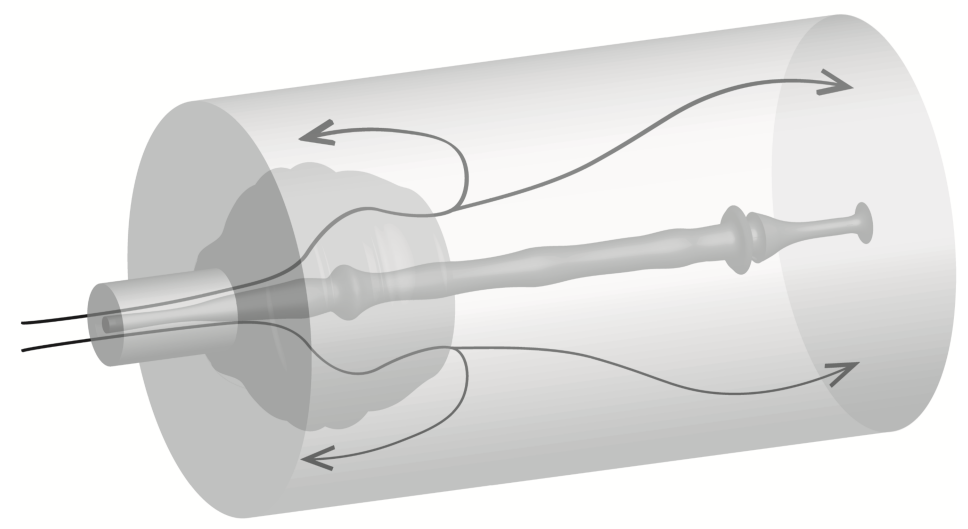

Figure 3.1: Optimisation of ANG tanks through the addition of non-adsorbent materials.

The gain in gas adsorbed obtained in an optimised tank (opt) in relation to a tank constituted purely by adsorbent material (pure) is represented by $\gamma$ and denotes the increment in the percentage of mass adsorbed. It is correlated to the variation of adsorbent volume inside the tank by

$$
\left(1+\gamma_{a}\right)=\frac{Q_{e q}\left(\overline{P_{f, o p t}}, \overline{T_{f, \text { opt }}}\right)-Q_{e q}\left(P_{a t m}, T_{a t m}\right)}{Q_{e q}\left(\overline{P_{f, p u r e}}, \overline{T_{f, p u r e}}\right)-Q_{e q}\left(P_{a t m}, T_{a t m}\right)}\left(1-\frac{V_{\text {non }}}{V_{\text {tank }}}\right) .
$$

Figure 3.2 illustrates the case of a tank which is presenting a final average temperature of $323 \mathrm{~K}$ at the end of the adsorption cycle. It is shown how much volume is possible to occupy with non-adsorbent materials according to the reduction of the temperature in order to achieved certain gain in the mass of gas adsorbed. For instance, if the replacement of $5 \%$ of the adsorbent bed with some non-adsorbent material leads to the reduction of final average temperature to $300 \mathrm{~K}$, it would be possible to adsorb $30 \%$ more gas.

\subsection{Manufacturing Considerations}

According to Menon \& Komarneni (1998), the packing density of adsorbent particles is a key factor for the overall capacity of gas storage in ANG systems. The most promising 


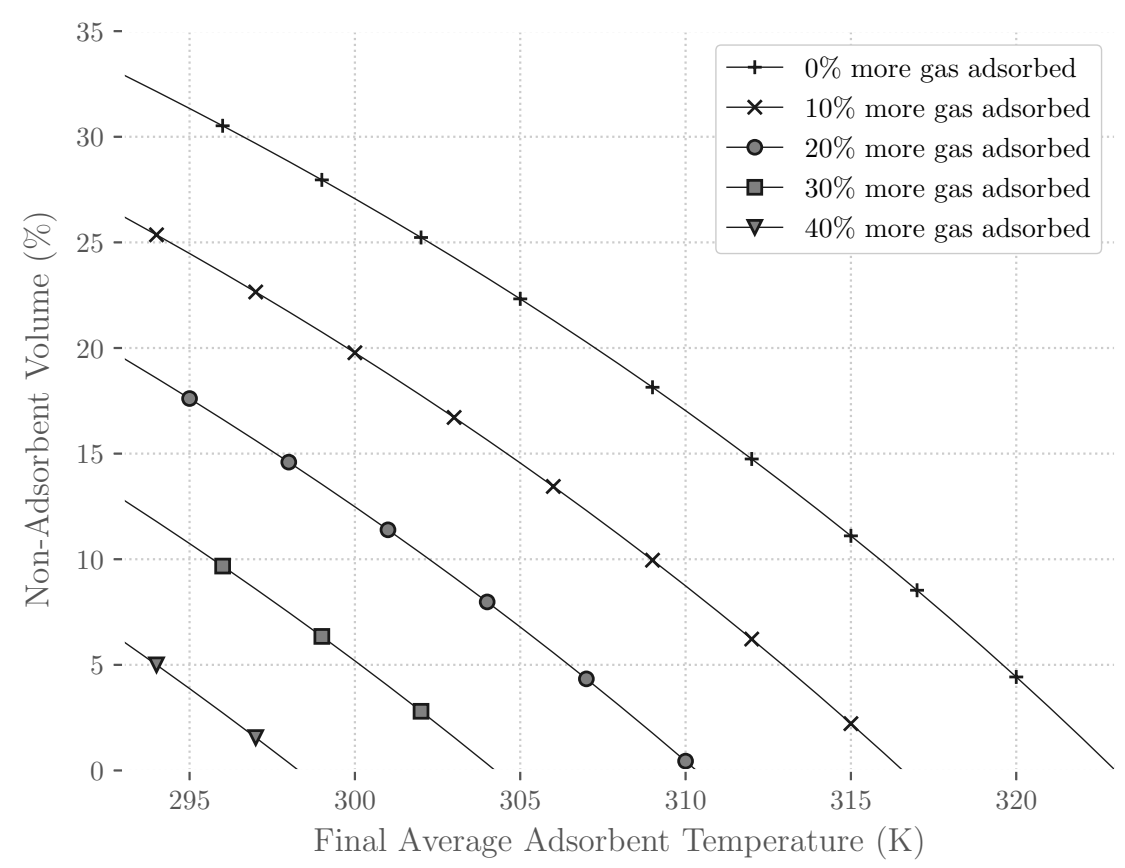

Figure 3.2: Isomassic curves given by the variation of adsorbent temperature and relative non-adsorbent volume in adsorption cycle.

approaches involve the fabrication of monolithic carbon based on the extrusion, cold pressing or hot isostatic pressing of the activated carbon powder associated with some binder. As a monolith, the void volume between particles is reduced and, therefore, the relative volume of micropores with respect to the total vessel volume is increased, resulting in more gas being adsorbed (QUINN et al., 1994). The fabrication method described in Bose et al. (1991) comprises mixing high surface area carbon particles with a solution of a binder in a solvent, forming a slurry. The use of binders, typically thermoplastic polymers, is important to prevent future powdering of the adsorbent bed when subjected to vibration or strong gas flow. The solvent is then removed, resulting in binder-coated carbon particles, which are compressed to form a compacted adsorbent mass of any desired geometric shape (BOSE et al., 1991).

Hence, ANG tanks can present features of any complexity without prejudice to the manufacturing process. Non-adsorbent parts, can be manufactured through traditional methods, such as machining or casting, and assembled at the desired position inside the tanks. The binder-coated carbon particles can then be poured into segments of the tank and compressed, occupying the whole void space left. 


\subsection{Objective Function}

The optimisation of ANG storing systems can be addressed by focusing on several different objectives. Figure 3.3 illustrates the typical variation of the adsorbed gas mass, pressure and temperature of an ANG tank serving as an on-demand source of fuel, such as in gas-powered vehicles or in residences. Starting from the average density of total gas at the equilibrium, $\overline{\left(Q+\rho_{g}\right)_{i n i, a}}$, the tank admits gas while pressure and temperature increase during the filling cycle, reaching the final average total density of $\overline{\left(Q+\rho_{g}\right)_{\text {fin }, a}}$. Such average density is conserved inside the tank until its future depletion. While the tank is kept quiescent, its temperature slowly approaches the equilibrium with the atmospheric temperature, $T_{a t m}$, causing its internal pressure to drop due to the gas phase change from gaseous to adsorbed. The stored gas is then delivered through a nearly isothermal desorption, maintaining equilibrium with $T_{a t m}$ during the whole emptying cycle.

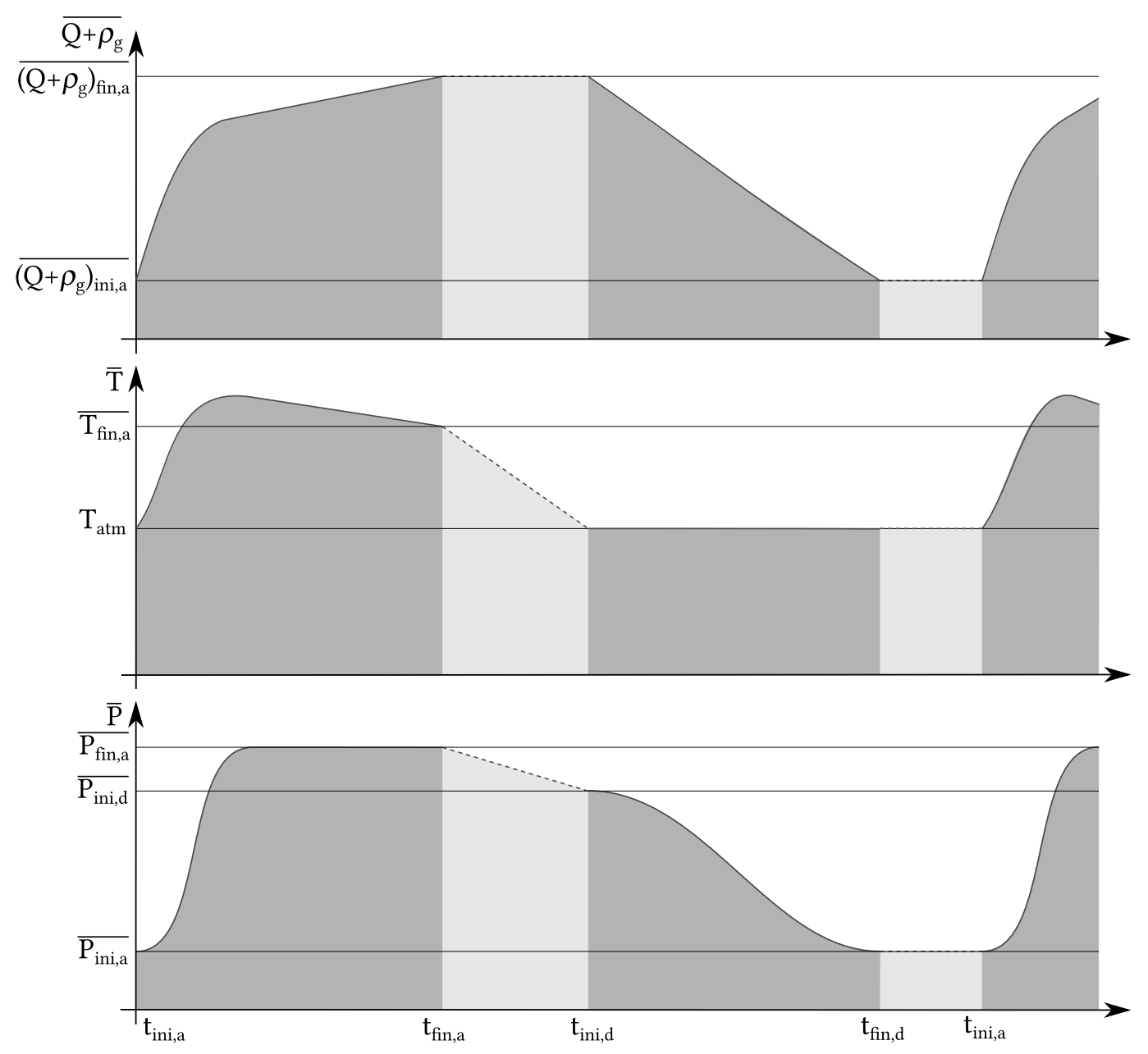

Figure 3.3: Cycle considered for the optimisation of ANG tanks applied to on-demand consumption.

The goal when designing such tanks is to maximise the total intake of gas during the 
filling cycle by mitigating the rise of the adsorbent bed temperature. The optimisation problem consists of

$$
\begin{aligned}
\text { Maximise }: & J_{a}(\mathrm{e})=\left[\int_{\Omega}\left(Q+\rho_{g}\right) d x\right]_{t_{\text {ini }, a}}^{t_{f i n, a}}, \\
\text { subjected to }: & 0 \leq \mathrm{e} \leq 1, \\
& \mathbb{M}(P, T, Q)+\mathbb{E}(P, T, Q)+\mathbb{D}(P, T, Q)=0 .
\end{aligned}
$$

\subsection{Material Model}

The design variables are defined as pseudo-porosities representing two materials: adsorbent and non-adsorbent. Given the poor conductivity presented by typical adsorbents, suitable non-adsorbent materials present relatively high conductivity or heat capacity, aiming to improve the heat transfer of the system.

The alternation between adsorbent (ads) and non-adsorbent (non) materials through the domain can be expressed by a pseudo-porosity e as

$$
\mathbb{P}(\mathrm{e})= \begin{cases}\mathbb{P}_{\text {non }}, & \text { if } \mathrm{e}=0 \\ \mathbb{P}_{\text {ads }}, & \text { if } \mathrm{e}=1\end{cases}
$$

where the actual property $\mathbb{P}$ is given by $\mathbb{P}_{\text {ads }}$ or $\mathbb{P}_{\text {non }}$ depending on e value.

\subsubsection{Material Models with Penalisation}

A binary law of material defines an ill-posed problem, with multiple local minima. Such issue can be avoided by relaxing the definition and assuming intermediate values, ranging from 0 to 1 . Since only 0 and 1 represent existent materials, it is desirable to recover the discrete nature of this material law. The Solid Isotropic Material with Penalisation (SIMP) (BENDSØE; SIGMUND, 1999) addresses this problem by including a penalisation coefficient $p$, resulting in

$$
\mathbb{P}(\mathrm{e})=\left(1-\mathrm{e}^{p}\right) \mathbb{P}_{\text {non }}+\left(\mathrm{e}^{p}\right) \mathbb{P}_{a d s}
$$

For structural cases, where $\mathbb{P}_{\text {non }} \equiv \mathbb{P}_{\text {void }}$ and $\mathbb{P}_{\text {ads }} \equiv \mathbb{P}_{\text {solid }}$, Bendsøe \& Sigmund (1999) recalls that the interpolated material property should not exceed the Hashin-Shtrikman upper bound for any value of the design variable e. In order to meet this requirement it is sufficient that $p \geq 3$. However, such penalisation creates multiple local minima and 
makes the final topology very dependent on the initial guess (BENDSØE; SIGMUND, 1999). Thus, a continuation scheme is advisable, by which the penaliser $p$ is slowly increased through the computations.

Figure 3.4 shows the impact of the penalisation coefficient to the property being interpolated by SIMP. Taking the pair Steel-Carbon as an example, it can be seen that the resulting property for intermediate values of the design variable, e, tends to Steel $\left(k=47.00 \mathrm{Wm}^{-1} \mathrm{~K}^{-1}\right.$ and $\left.W=0.00 \mathrm{~cm}^{3} \mathrm{~g}^{-1}\right)$ as $p \rightarrow \infty$, while it tends to Activated Carbon $\left(k=0.21 \mathrm{Wm}^{-1} \mathrm{~K}^{-1}\right.$ and $\left.W=0.33 \mathrm{~cm}^{3} \mathrm{~g}^{-1}\right)$ as $p \rightarrow 0$.

Borrvall \& Petersson (2003) introduced a scheme more suitable to deal with the interpolation of permeabilities, which are separated by several orders of magnitude and would require extremely high values for $p$. This material model is based on the inverse permeability, $S$, and it is given by

$$
S(\mathrm{e})=\frac{1}{K(\mathrm{e})}=S_{n o n}+\left(S_{a d s}-S_{n o n}\right) \frac{1+p}{\mathrm{e}+p} \mathrm{e}
$$

where $S_{a d s}$ and $S_{n o n}$ are given by

$$
S_{[a d s, n o n]}=\frac{1}{K_{[a d s, n o n]}} .
$$

The adsorbent permeability $\left(K_{a d s}\right)$ is in the order of $1.00 \times 10^{-10} \mathrm{~m}^{2}$ (SAHOO et al., 2011), while the non-adsorbent $\left(K_{\text {non }}\right)$ material must be an impermeable medium, in which the permeability should be as low as $1.00 \times 10^{-22} \mathrm{~m}^{2}$. For such properties, the resulting permeability based on Borrvall's scheme is shown in Figure 3.5.

\subsubsection{Offset Hyperbolic Tangents (OHT) Material Model}

SIMP and Borrvall's models present some disadvantages when dealing with discrepant properties, such as the interpolation from $K_{a d s}=1.00 \times 10^{-10} \mathrm{~m}^{2}$ to $K_{\text {non }}=1.00 \times 10^{-22} \mathrm{~m}^{2}$. A material model based on hyperbolic tangents is able to offer a better discrimination of materials, being defined as

$$
\mathbb{P}(\mathrm{e})=(1-m(\mathrm{e})) \mathbb{P}_{n o n}+m(\mathrm{e}) \mathbb{P}_{a d s}
$$

in which

$$
m(\mathrm{e})=\frac{\tanh \left(p\left(\mathrm{e}-\mathrm{e}_{c}\right)\right)+1}{2},
$$

where the transition from one material to the other is centred on the value $\mathrm{e}_{c}$. 


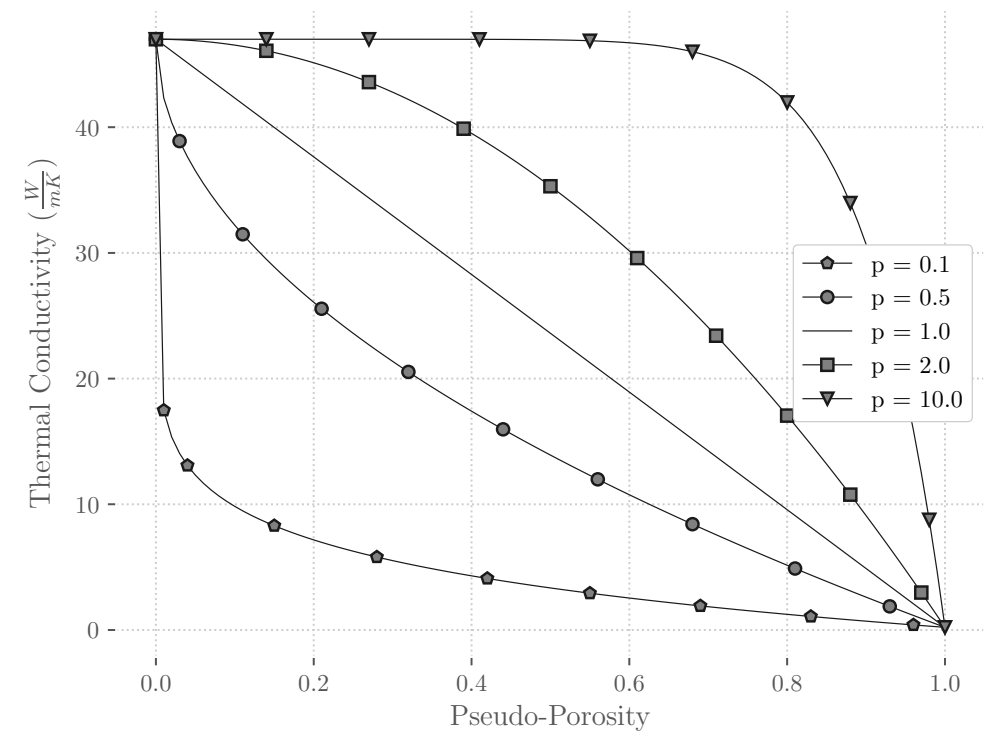

(a) Thermal Conductivity $(k)$

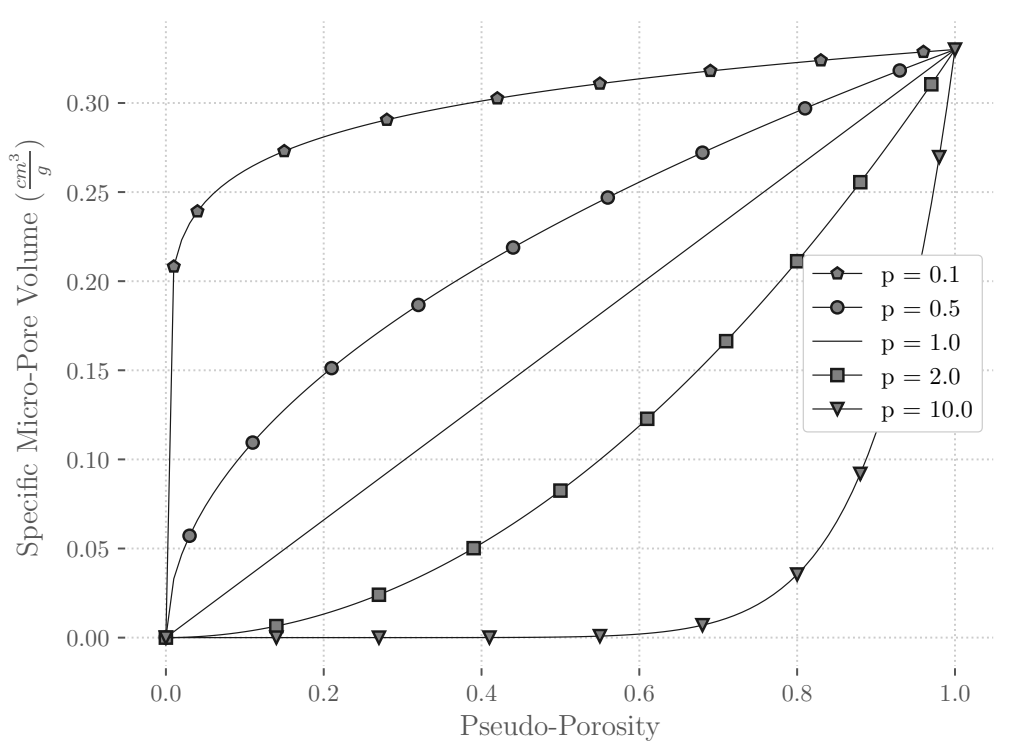

(b) Specific Micro-Pore Volume $(W)$

Figure 3.4: SIMP for Steel and Carbon properties.

In the case of Steel-Carbon pair, it can be seen that the hyperbolic tangent approach is suitable to interpolate all the material properties with its generic form, as seen in Figure 3.6 .

When optimising adsorption systems, materials presenting intermediate properties often constitute the best solution from the optimiser's perspective. For instance, in the case of seeking an optimised placement of Steel on a Carbon bed, the conductivity of 


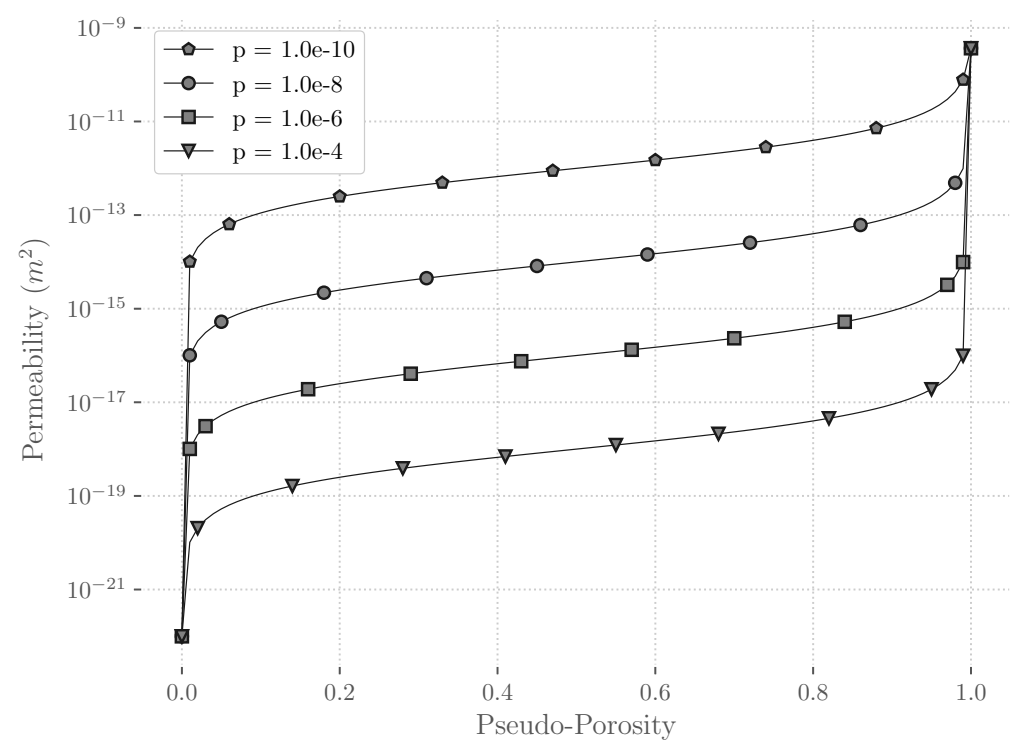

Figure 3.5: Borrvall's material model for Steel and Carbon Permeability $(K)$.

the non-adsorbent material is more than 200 times larger than adsorbent's and Steel adsorption capacity is null. Therefore, a material presenting half of Steel's conductivity and half of Carbon's adsorption would be a good choice, if feasible.

It is then necessary to adopt a material model which forces the optimiser to clearly choose one or other material. The material model based on hyperbolic tangents presented in Eq. (3.11) is suitable for this sort of requirement. However, when choosing the material change centred on the same $e_{c}$ for all the properties, the existence of hybrid materials prevails despite the penalisation imposed, as shown in Figure 3.7a. Such issue is solved by defining different values of $\mathrm{e}_{c}$ for different properties. For the property $A$, where the functional of interest $J\left(A_{a d s}\right)$ (adsorbent property in the whole domain) is higher than $J\left(A_{n o n}\right)$ (non-adsorbent property in the whole domain), the phase change is centred on $\mathrm{e}_{c}=0.5+\epsilon$. Conversely, for a property $B$ which presents $J\left(B_{\text {non }}\right)$ higher than $J\left(B_{\text {ads }}\right)$, $\mathrm{e}_{c}=0.5-\epsilon$ is adopted. In the absence of an offset, there would be intermediate properties at $\mathrm{e}=0.5$ regardless of the penalisation value. Figure 3.7 illustrates such material model for the offset $\epsilon=0.02$. The function $m(\mathrm{e})$ in the Offset Hyperbolic Tangents (OHT) material model is then given by

$$
m(\mathrm{e})=\frac{\tanh (p(\mathrm{e}+s \epsilon-0.5))+1}{2},
$$


where

$$
s=\operatorname{sgn}\left(J\left(\mathbb{P}_{\text {non }}\right)-J\left(\mathbb{P}_{\text {ads }}\right)\right),
$$

with sgn denoting the signum function.

A comparison between OHT and traditional material models is presented in Chapter 5, highlighting its advantages for the problems studied in this work. In the next Chapter, the numerical implementation of the adsorption problem and the topology optimisation algorithm is detailed, along with the necessary verifications. 


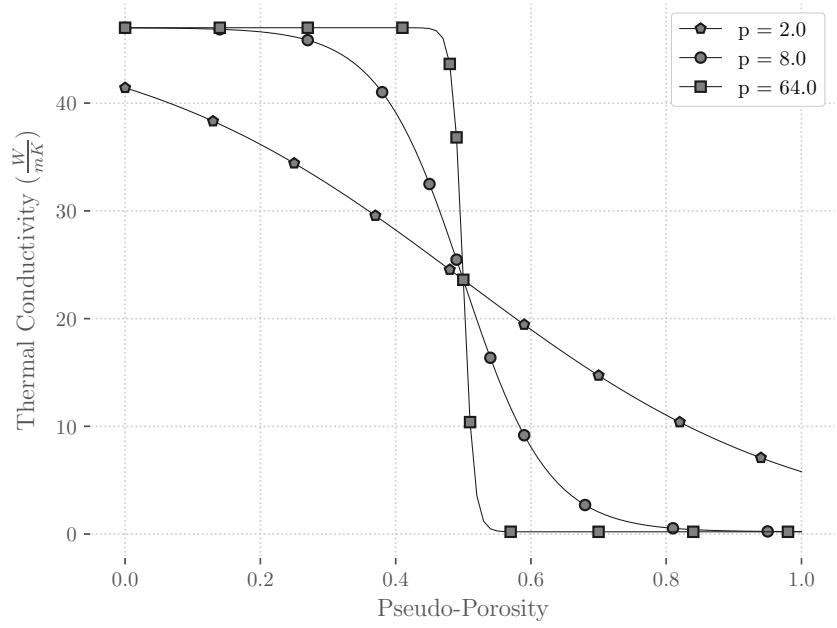

(a) Thermal Conductivity $(k)$

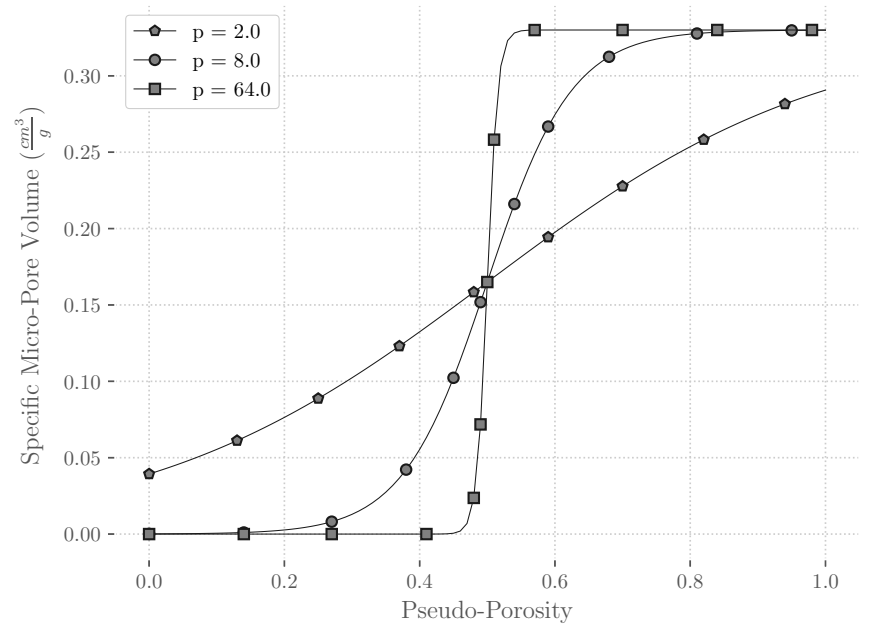

(b) Specific Micro-Pore Volume $(W)$

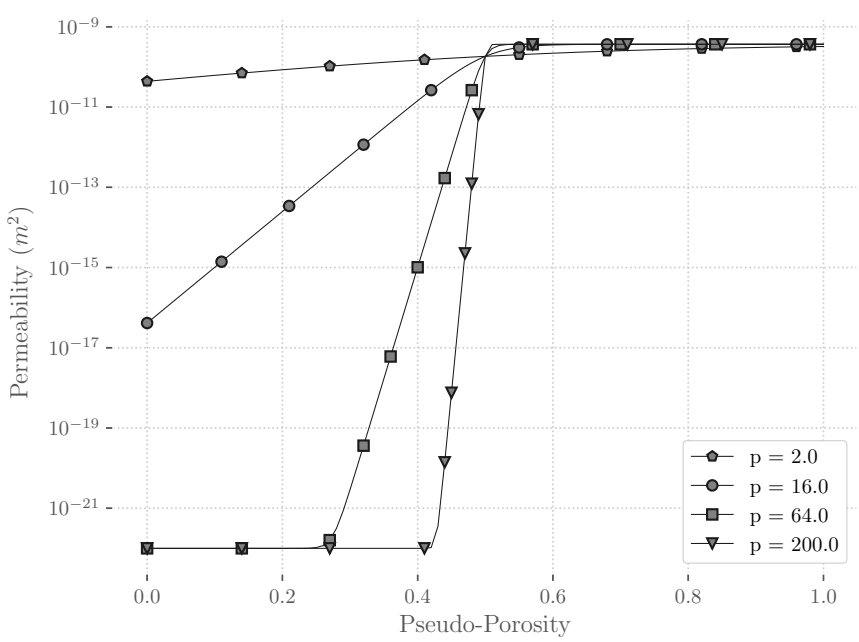

(c) Permeability $(K)$

Figure 3.6: Hyperbolic tangent material model for Steel and Carbon properties with $e_{c}=0.5$. 


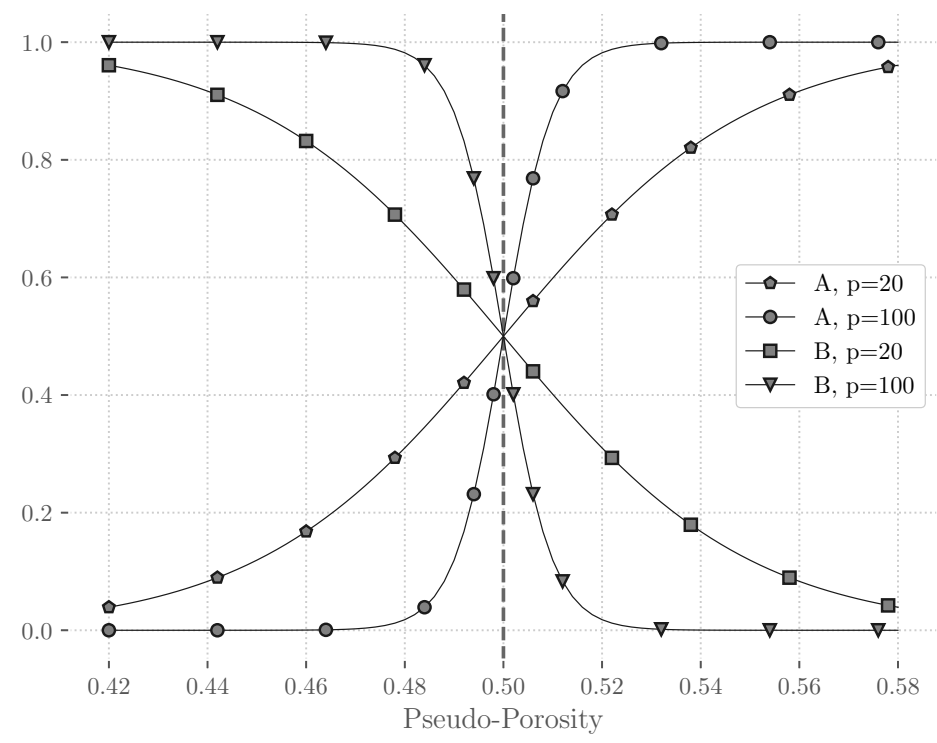

(a) $\mathrm{e}_{c}=0.5$ for $\mathrm{A}$ and $\mathrm{B}$

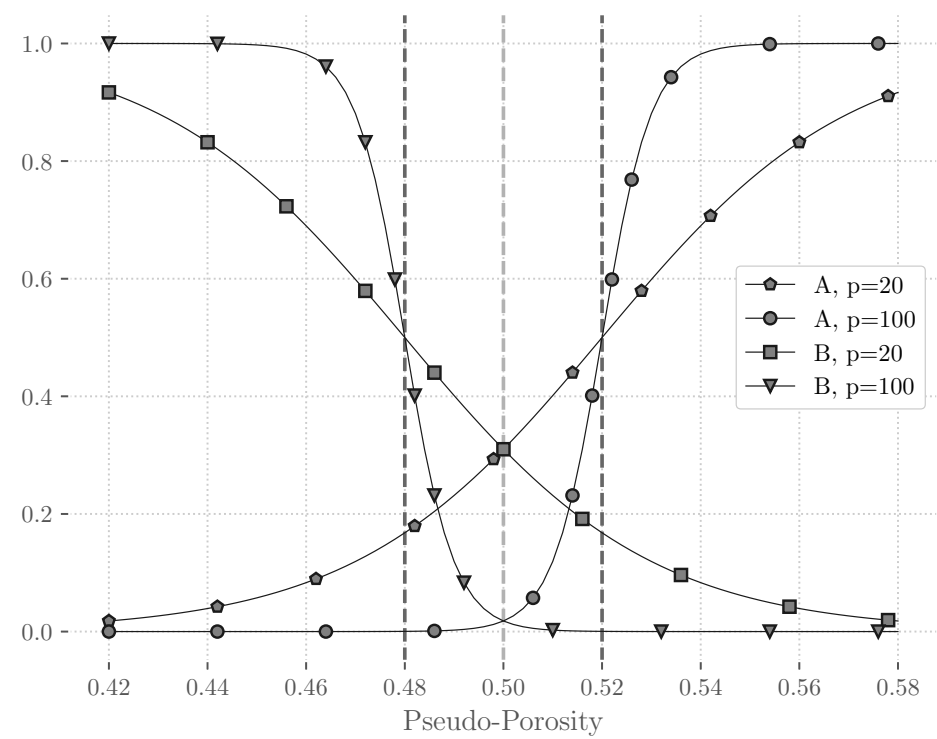

(b) $\mathrm{e}_{c}=0.52$ for $\mathrm{A}$ and $\mathrm{e}_{c}=0.48$ for $\mathrm{B}$

Figure 3.7: Material model for properties A and B. 


\section{Numerical Implementation and Verification}

In this Chapter, the discretisation of the weak forms is presented in detail and an adjoint system is derived in view of the implementation of a gradient-based topology optimisation. The solution of the adsorption problem and the optimisation algorithm are then described along with several numerical verifications.

\subsection{Discrete Adsorption Problem}

The weak forms of the adsorption problem derived in Section 2.4 are approximated over a finite element, $\omega$, by (REDDY; GARTLING, 2010)

$$
\begin{gathered}
P(\mathbf{x}, t) \cong \sum_{n=1}^{N} \phi_{n}(\mathbf{x}) P_{n}(t)=\mathbf{\Phi}^{T} \mathbf{P} \\
T(\mathbf{x}, t) \cong \sum_{m=1}^{M} \psi_{m}(\mathbf{x}) T_{m}(t)=\mathbf{\Psi}^{T} \mathbf{T},
\end{gathered}
$$

where $\mathbf{P}$ and $\mathbf{T}$ are, respectively, the vectors with the nodal values of pressure and temperature, $N$ and $M$ denotes the number of nodes used for each unknown and $\phi$ and $\psi$ are the shape functions, which are assembled in matrices $\boldsymbol{\Phi}$ and $\boldsymbol{\Psi}$.

Analogously, the mass of adsorbed gas per volume of adsorbent $(Q)$ is interpolated over finite elements by the shape function $\xi$ as

$$
Q(\mathbf{x}, t) \cong \sum_{l=1}^{L} \xi_{l}(\mathbf{x}) Q_{l}(t)=\mathbf{\Xi}^{T} \mathbf{Q}
$$


The interpolation of the total porosity $(\varepsilon)$ and all other material properties $\left(\rho_{s}, C p_{s}\right.$, $K_{s}$ and $K_{s}$ ) is also done by using $\xi$, which is given by

$$
\varepsilon(\mathbf{x}, t) \cong \sum_{l=1}^{L} \xi_{l}(\mathbf{x}) \varepsilon_{l}(t)=\mathbf{\Xi}^{T} \boldsymbol{\varepsilon} .
$$

Making $v_{M}=\phi, v_{E}=\psi$ and $v_{D}=\xi$, results in the discrete equations given by

$$
\begin{gathered}
\mathbf{W}(\mathbf{T}) \dot{\mathbf{P}}+\mathbf{S}(\mathbf{P}, \mathbf{T}) \dot{\mathbf{T}}+\mathbf{Y}_{\mathbf{M}} \dot{\mathbf{Q}}+\mathbf{Z}(\mathbf{P}, \mathbf{T}) \mathbf{P}=\mathbf{0} \\
\mathbf{J} \dot{\mathbf{P}}+\mathbf{N}(\mathbf{P}, \mathbf{T}, \mathbf{Q}) \dot{\mathbf{T}}+\mathbf{Y}_{\mathbf{E}} \dot{\mathbf{Q}}+\mathbf{L}(\mathbf{P}, \mathbf{T}) \mathbf{T}+\mathbf{K} \mathbf{T}=\mathbf{0} \\
\mathbf{Y}_{\mathbf{D}} \dot{\mathbf{Q}}+\mathbf{R}(\mathbf{T}) \mathbf{Q}=\mathbf{V}(\mathbf{P}, \mathbf{T}),
\end{gathered}
$$

which results in the following system:

$$
\begin{array}{r}
{\left[\begin{array}{ccc}
\mathbf{W}(\mathbf{T}) & \mathbf{S}(\mathbf{P}, \mathbf{T}) & \mathbf{Y}_{\mathbf{M}} \\
\mathbf{J} & \mathbf{N}(\mathbf{P}, \mathbf{T}, \mathbf{Q}) & \mathbf{Y}_{\mathbf{E}} \\
\mathbf{0} & \mathbf{0} & \mathbf{Y}_{\mathbf{D}}
\end{array}\right]\left\{\begin{array}{c}
\dot{\mathbf{P}} \\
\dot{\mathbf{T}} \\
\dot{\mathrm{Q}}
\end{array}\right\}} \\
+\left[\begin{array}{ccc}
\mathbf{Z}(\mathbf{P}, \mathbf{T}) & \mathbf{0} & \mathbf{0} \\
\mathbf{0} & \mathbf{L}(\mathbf{P}, \mathbf{T})+\mathbf{K} & \mathbf{0} \\
\mathbf{0} & \mathbf{0} & \mathbf{R}(\mathbf{T})
\end{array}\right]\left\{\begin{array}{c}
\mathbf{P} \\
\mathbf{T} \\
\mathbf{Q}
\end{array}\right\} \\
=\left\{\begin{array}{c}
\mathbf{0} \\
\mathbf{0} \\
\mathbf{V}(\mathbf{P}, \mathbf{T})
\end{array}\right\},
\end{array}
$$

whose matrices are assembled based on the coefficients defined in Appendix A.1.

The system given in Eq. (4.8) is rewritten for the $n$-th timestep as

$$
\mathbf{F}_{t}\left(\mathbf{w}_{n}\right) \dot{\mathbf{w}}_{n}+\mathbf{F}_{s}\left(\mathbf{w}_{n}\right) \mathbf{w}_{n}=\mathbf{F}_{r}\left(\mathbf{w}_{n}\right)
$$

where the state variable $\mathbf{w}_{n}$, constituted by pressure, temperature and adsorbed gas, is given by

$$
\mathbf{w}_{n}=\left\{\begin{array}{c}
\mathbf{P}_{n} \\
\mathbf{T}_{n} \\
\mathbf{Q}_{n}
\end{array}\right\}
$$

The discretisation of the equations in time by using backward Euler method results 
in a lower-triangular system given by

$$
\left[\begin{array}{ccccc}
\mathbf{I} & \mathbf{0} & \cdots & \mathbf{0} & \mathbf{0} \\
\mathbf{C}_{P}\left(\mathbf{w}_{0}\right) & \mathbf{C}_{C}\left(\mathbf{w}_{1}\right) & \cdots & \mathbf{0} & \mathbf{0} \\
\vdots & \vdots & \ddots & \vdots & \vdots \\
\mathbf{0} & \mathbf{0} & \cdots & \mathbf{C}_{P}\left(\mathbf{w}_{N-1}\right) & \mathbf{C}_{C}\left(\mathbf{w}_{N}\right)
\end{array}\right]\left\{\begin{array}{c}
\mathbf{w}_{0} \\
\mathbf{w}_{1} \\
\vdots \\
\mathbf{w}_{N-1} \\
\mathbf{w}_{N}
\end{array}\right\}=\left\{\begin{array}{c}
\mathbf{w}_{i n i} \\
\mathbf{F}_{r}\left(\mathbf{w}_{1}\right) \\
\vdots \\
\mathbf{F}_{r}\left(\mathbf{w}_{N-1}\right) \\
\mathbf{F}_{r}\left(\mathbf{w}_{N}\right)
\end{array}\right\}
$$

where $\mathbf{W}_{\text {ini }}$ denotes the initial state conditions and

$$
\begin{gathered}
\mathbf{C}_{\mathbf{P}}\left(\mathbf{w}_{n}\right)=-\frac{\mathbf{F}_{t}\left(\mathbf{w}_{n}\right)}{\Delta t} \\
\mathbf{C}_{\mathbf{C}}\left(\mathbf{w}_{n}\right)=\frac{\mathbf{F}_{t}\left(\mathbf{w}_{n}\right)}{\Delta t}+\mathbf{F}_{s}\left(\mathbf{w}_{n}\right) .
\end{gathered}
$$

\subsection{Discrete Adjoint System for the Adsorption Prob- lem}

In the optimisation problem given in Eq. (3.6), the functional of interest $J(\mathbf{w}(\mathbf{e}), \mathbf{e})$ is constrained to the PDE system $\mathbf{F}(\mathbf{w}, \mathbf{e})=\mathbf{0}$ given in Eq. (4.11). Such problem is expressed by the following augmented Lagrangian as

$$
J=\int_{t_{i n i}}^{t f i n}\left(\delta_{t_{f i n}}-\delta_{t_{i n i}}\right) B+\boldsymbol{\lambda}^{T}\left(\mathbf{F}_{t} \dot{\mathbf{w}}+\mathbf{F}_{s} \mathbf{w}-\mathbf{F}_{r}\right) d t
$$

where $B$ denotes the total gas mass, given by:

$$
\int_{\Omega}\left(Q+\rho_{g}\right) d x
$$

and, for simplicity

$$
\delta_{t_{f i n}}-\delta_{t_{i n i}}=\delta\left(t-t_{f i n}\right)-\delta\left(t-t_{i n i}\right),
$$

where $\delta$ denotes Dirac's delta operator, making the term $\left(\delta_{t_{f i n}}-\delta_{t_{i n i}}\right) B$ to be equal to the difference in the total gas mass between the beginning and the end of the adsorption cycle.

The vector of Lagrange multipliers, $\boldsymbol{\lambda}$, is given by

$$
\boldsymbol{\lambda}=\left\{\begin{array}{c}
\boldsymbol{\lambda}_{M} \\
\boldsymbol{\lambda}_{E} \\
\boldsymbol{\lambda}_{D}
\end{array}\right\}
$$


The derivative of the reduced functional $J$ in respect to a design variable $e_{i}$ is given by

$$
\begin{array}{r}
\frac{d J}{d e_{i}}=\int_{t_{i n i}}^{t_{f i n}}\left(\delta_{t_{f i n}}-\delta_{t_{i n i}}\right)\left(\frac{\partial B}{\partial e_{i}}+\frac{\partial B}{\partial \mathbf{w}} \frac{d \mathbf{w}}{d e_{i}}\right) d t \\
+\int_{t_{\text {ini }}}^{t_{f i n}} \boldsymbol{\lambda}^{T}\left(\left(\frac{\partial \mathbf{F}_{t}}{\partial e_{i}}+\frac{\partial \mathbf{F}_{t}}{\partial \mathbf{w}} \frac{d \mathbf{w}}{d e_{i}}\right) \dot{\mathbf{w}}\right. \\
+\mathbf{F}_{t} \frac{d \dot{\mathbf{w}}}{d e_{i}}+\left(\frac{\partial \mathbf{F}_{s}}{\partial e_{i}}+\frac{\partial \mathbf{F}_{s}}{\partial \mathbf{w}} \frac{d \mathbf{w}}{d e_{i}}\right) \mathbf{w} \\
\left.+\mathbf{F}_{s} \frac{d \mathbf{w}}{d e_{i}}-\frac{\partial \mathbf{F}_{r}}{\partial e_{i}}-\frac{\partial \mathbf{F}_{r}}{\partial \mathbf{w}} \frac{d \mathbf{w}}{d e_{i}}\right) d t
\end{array}
$$

Integrating the term involving $d \dot{\mathbf{w}} / d e_{i}$ by parts, it results in

$$
\frac{d J}{d e_{i}}=\Theta_{s e n}+\Theta_{b, i n i}+\Theta_{b, f i n}+\Theta_{a d j}
$$

where

$$
\begin{gathered}
\Theta_{\text {sen }}=\int_{t_{\text {ini }}}^{t_{f i n}}\left(\delta_{t_{f i n}}-\delta_{t_{\text {ini }}}\right) \frac{\partial B}{\partial e_{i}}+\boldsymbol{\lambda}^{T}\left(\frac{\partial \mathbf{F}_{t}}{\partial e_{i}} \dot{\mathbf{w}}+\frac{\partial \mathbf{F}_{s}}{\partial e_{i}} \mathbf{w}-\frac{\partial \mathbf{F}_{r}}{\partial e_{i}}\right) d t \\
\Theta_{b, i n i}=-\left.\boldsymbol{\lambda}^{T} \mathbf{F}_{t} \frac{d \mathbf{w}}{d e_{i}}\right|_{t_{\text {ini }}} \\
\Theta_{b, f i n}=\left.\boldsymbol{\lambda}^{T} \mathbf{F}_{t} \frac{d \mathbf{w}}{d e_{i}}\right|_{t_{f i n}} \\
\Theta_{a d j}=\int_{t_{i n i}}^{t_{f i n}} \frac{d \mathbf{w}}{d e_{i}}\left[\left(\delta_{t_{f i n}}-\delta_{t_{i n i}}\right) \frac{\partial B}{\partial \mathbf{w}}-\frac{\partial\left(\boldsymbol{\lambda}^{T} \mathbf{F}_{t}\right)}{\partial t}+\boldsymbol{\lambda}^{T}\left(\mathbf{F}_{s}+\frac{\partial \mathbf{F}_{t}}{\partial \mathbf{w}} \dot{\mathbf{w}}+\frac{\partial \mathbf{F}_{s}}{\partial \mathbf{w}} \mathbf{w}-\frac{\partial \mathbf{F}_{r}}{\partial \mathbf{w}}\right)\right] d t
\end{gathered}
$$

Since the term $d \mathbf{w} / d e_{i}$ is unknown for $t>t_{i n i}$, the terms $\Theta_{b, f i n}$ and $\Theta_{\text {adj }}$ cannot be evaluated explicitly. Given that $\boldsymbol{\lambda}$ is arbitrary, it can be chosen in order to nullify these terms (DAHL et al., 2008). To ensure $\Theta_{b, f i n}=0$ it is necessary to choose $\boldsymbol{\lambda}$ such that $\boldsymbol{\lambda}\left(t_{f i n}\right)=\mathbf{0}$. In adsorption systems, although $P_{i n i}$ and $T_{i n i}$ are imposed and do not depend on the design variables, the initial density of gas adsorbed, $Q_{i n i}$, is dependent on the volume of adsorbent material inside the domain. The derivative of the state variable in respect to $e_{i}$ is given by

$$
\left.\frac{d \mathbf{w}}{d e_{i}}\right|_{t_{i n i}}=\left\{\begin{array}{c}
0 \\
0 \\
Q_{e q}\left(P_{i n i}, T_{i n i}\right) \frac{d m\left(e_{i}\right)}{d e_{i}}
\end{array}\right\}
$$

where the equilibrium adsorbed density $Q_{e q}$ is given in Eq. (2.24) and the material model interpolation function $m\left(e_{i}\right)$ is given in Eq. (3.13).

The term $\Theta_{a d j}$ is null when the expression between brackets is equal to zero, which 
can be rewritten as

$$
\left(\delta_{t_{f i n}}-\delta_{t_{i n i}}\right) \frac{\partial B}{\partial \mathbf{w}}-\dot{\boldsymbol{\lambda}}^{T} \mathbf{F}_{t}-\boldsymbol{\lambda}^{T} \frac{\partial \mathbf{F}_{t}}{\partial \mathbf{w}} \frac{d \mathbf{w}}{d t}+\boldsymbol{\lambda}^{T}\left(\mathbf{F}_{s}+\frac{\partial \mathbf{F}_{t}}{\partial \mathbf{w}} \frac{d \mathbf{w}}{d t}+\frac{\partial \mathbf{F}_{s}}{\partial \mathbf{w}} \mathbf{w}-\frac{\partial \mathbf{F}_{r}}{\partial \mathbf{w}}\right)=0
$$

resulting in

$$
\left(\delta_{t_{f i n}}-\delta_{t_{i n i}}\right) \frac{\partial B}{\partial \mathbf{w}}-\dot{\boldsymbol{\lambda}}^{T} \mathbf{F}_{t}+\boldsymbol{\lambda}^{T}\left(\mathbf{F}_{s}+\frac{\partial \mathbf{F}_{s}}{\partial \mathbf{w}} \mathbf{w}-\frac{\partial \mathbf{F}_{r}}{\partial \mathbf{w}}\right)=0
$$

The Lagrange multiplier $\boldsymbol{\lambda}$ is obtained by the solution of the adjoint problem given by

$$
\begin{array}{r}
\left(\mathbf{F}_{t}(\mathbf{w})\right)^{T} \dot{\boldsymbol{\lambda}}(t)-\left(\mathbf{F}_{s}(\mathbf{w})+\frac{\partial \mathbf{F}_{s}(\mathbf{w})}{\partial \mathbf{w}} \mathbf{w}-\frac{\partial \mathbf{F}_{r}(\mathbf{w})}{\partial \mathbf{w}}\right)^{T} \boldsymbol{\lambda}(t)=\left(\delta_{t_{f i n}}-\delta_{t_{i n i}}\right) \\
\boldsymbol{\lambda}\left(t_{f i n}\right)=\mathbf{0}
\end{array}
$$

Such system poses a terminal value problem, which can be converted in an initial value problem by substituting the time variable $t$ per $\tau=t_{\text {fin }}-t$. A new system is then defined in terms of $\overline{\boldsymbol{\lambda}}(\tau)=\boldsymbol{\lambda}\left(t_{f i n}-t\right)$, given by

$$
\begin{array}{r}
\left(\mathbf{F}_{t}(\mathbf{w})\right)^{T} \dot{\bar{\lambda}}(\tau)+\left(\mathbf{F}_{u}(\mathbf{w})\right)^{T} \overline{\boldsymbol{\lambda}}(\tau)=\left(\delta_{\tau_{f i n}}-\delta_{\tau_{i n i}}\right) \frac{\partial B}{\partial \mathbf{w}}, \\
\overline{\boldsymbol{\lambda}}\left(\tau_{\text {ini }}\right)=\mathbf{0},
\end{array}
$$

where, for simplicity

$$
\mathbf{F}_{u}(\mathbf{w})=\mathbf{F}_{s}(\mathbf{w})+\frac{\partial \mathbf{F}_{s}(\mathbf{w})}{\partial \mathbf{w}} \mathbf{w}-\frac{\partial \mathbf{F}_{r}(\mathbf{w})}{\partial \mathbf{w}}
$$

Let the Laplace transform of $\overline{\boldsymbol{\lambda}}(\tau)$ to be expressed as

$$
\mathcal{L}\{\bar{\lambda}(\tau)\}=\bar{\Lambda}(s)
$$

The Laplace transformation of each term in the Eq. (4.28) results in

$$
\begin{gathered}
\mathcal{L}\left\{\left(\mathbf{F}_{t}(\mathbf{w})\right)^{T} \dot{\bar{\lambda}}(\tau)\right\}=\left(\mathbf{F}_{t}(\mathbf{w})\right)^{T}\left(s \overline{\mathbf{\Lambda}}(s)-\overline{\boldsymbol{\lambda}}\left(\tau_{\text {ini }}\right)\right) \\
\mathcal{L}\left\{\left(\mathbf{F}_{u}(\mathbf{w})\right)^{T} \overline{\boldsymbol{\lambda}}(\tau)\right\}=\left(\mathbf{F}_{u}(\mathbf{w})\right)^{T} \bar{\Lambda}(s),
\end{gathered}
$$


$\mathcal{L}\left\{\left(\delta_{\tau_{f i n}}-\delta_{\tau_{i n i}}\right) \frac{\partial B}{\partial \mathbf{w}}\right\}=\int_{0}^{\infty} e^{-s \tau}\left(\delta_{\tau_{f i n}}-\delta_{\tau_{i n i}}\right) \frac{\partial B}{\partial \mathbf{w}} d \tau=\left(e^{-s \tau_{f i n}}-e^{-s \tau_{i n i}}\right) \mathcal{L}\left\{\frac{\partial B}{\partial \mathbf{w}}\right\}$.

Following, the inverse Laplace transform must be performed to go back to the reverse time domain $\tau$, which for the last term is given by

$\mathcal{L}^{-1}\left\{\left(e^{-s \tau_{f i n}}-e^{-s \tau_{i n i}}\right) \mathcal{L}\left\{\frac{\partial B(\tau)}{\partial \mathbf{w}}\right\}\right\}=H\left(\tau-\tau_{f i n}\right) \frac{\partial B\left(\tau-\tau_{f i n}\right)}{\partial \mathbf{w}}-H\left(\tau-\tau_{\text {ini }}\right) \frac{\partial B\left(\tau-\tau_{\text {ini }}\right)}{\partial \mathbf{w}}$

where $H$ denotes the Heaviside step function, which is applied to the function $B$ shifted by $\tau_{f i n}$ and $\tau_{i n i}$.

The adjoint problem in Eq. (4.28) is then given by

$$
\begin{array}{r}
\left(\mathbf{F}_{t}(\mathbf{w})\right)^{T} \dot{\bar{\lambda}}(\tau)+\left(\mathbf{F}_{u}(\mathbf{w})\right)^{T} \overline{\boldsymbol{\lambda}}(\tau)=H_{\tau_{f i n}} \frac{\partial B_{\tau_{f i n}}}{\partial \mathbf{w}}-H_{\tau_{i n i}} \frac{\partial B_{\tau_{i n i}}}{\partial \mathbf{w}}, \\
\overline{\boldsymbol{\lambda}}\left(\tau_{i n i}\right)=\mathbf{0},
\end{array}
$$

where, for simplicity

$$
H_{\tau_{f i n}} \frac{\partial B_{\tau_{f i n}}}{\partial \mathbf{w}}-H_{\tau_{i n i}} \frac{\partial B_{\tau_{i n i}}}{\partial \mathbf{w}}=H\left(\tau-\tau_{f i n}\right) \frac{\partial B\left(\tau-\tau_{f i n}\right)}{\partial \mathbf{w}}-H\left(\tau-\tau_{i n i}\right) \frac{\partial B\left(\tau-\tau_{i n i}\right)}{\partial \mathbf{w}} .
$$

The discretisation of the adjoint system in time via backward Euler method results in

$$
\left[\begin{array}{ccccc}
\mathbf{I} & \mathbf{0} & \cdots & \mathbf{0} & \mathbf{0} \\
\mathbf{A}_{P}\left(\mathbf{w}_{0}\right) & \mathbf{A}_{C}\left(\mathbf{w}_{1}\right) & \cdots & \mathbf{0} & \mathbf{0} \\
\vdots & \vdots & \ddots & \vdots & \vdots \\
\mathbf{0} & \mathbf{0} & \cdots & \mathbf{A}_{P}\left(\mathbf{w}_{N-1}\right) & \mathbf{A}_{C}\left(\mathbf{w}_{N}\right)
\end{array}\right]\left\{\begin{array}{c}
\overline{\boldsymbol{\lambda}}_{0} \\
\overline{\boldsymbol{\lambda}}_{1} \\
\vdots \\
\overline{\boldsymbol{\lambda}}_{N-1} \\
\overline{\boldsymbol{\lambda}}_{N}
\end{array}\right\}=\left\{\begin{array}{c}
\mathbf{0} \\
\mathbf{A}_{R}\left(\mathbf{w}_{1}\right) \\
\vdots \\
\mathbf{A}_{R}\left(\mathbf{w}_{N-1}\right) \\
\mathbf{A}_{R}\left(\mathbf{w}_{N}\right)
\end{array}\right\}
$$

where

$$
\begin{gathered}
\mathbf{A}_{P}\left(\mathbf{w}_{n}\right)=\left(-\frac{\mathbf{F}_{t}\left(\mathbf{w}_{n}\right)}{\Delta t}\right)^{T} \\
\mathbf{A}_{C}\left(\mathbf{w}_{n}\right)=\left(\frac{\mathbf{F}_{t}\left(\mathbf{w}_{n}\right)}{\Delta t}+\mathbf{F}_{s}\left(\mathbf{w}_{n}\right)+\frac{\partial \mathbf{F}_{s}\left(\mathbf{w}_{n}\right)}{\partial \mathbf{w}_{n}} \mathbf{w}_{n}-\frac{\partial \mathbf{F}_{r}\left(\mathbf{w}_{n}\right)}{\partial \mathbf{w}_{n}}\right)^{T} \\
\mathbf{A}_{R}\left(\mathbf{w}_{n}\right)=H_{\tau_{f i n}} \frac{\partial B_{\tau_{f i n}}}{\partial \mathbf{w}_{n}}-H_{\tau_{i n i}} \frac{\partial B_{\tau_{i n i}}}{\partial \mathbf{w}_{n}}
\end{gathered}
$$


The solution of the system above, in terms of $\bar{\lambda}(\tau)$, is then reversed in time to obtain $\boldsymbol{\lambda}(t)$. Therefore, the derivative of the functional $J$ in respect to $e_{i}$ is now known and given by

$$
\frac{d J}{d e_{i}}=\Theta_{s e n}+\Theta_{b, i n i}
$$

\subsection{Adsorption Problem Implementation}

Several open source software are available for computational fluid dynamics. Some examples are OpenFOAM (WELLER et al., 1998), based on the Finite Volume Method (FVM); FEniCS (LOGG, 2007) and Fluidity (PIGGOTT et al., 2008), based on Finite Element Method (FEM); SU2 (PALACIOS et al., 2013), able to handle both FVM and FEM; PyFR (WITHERDEN et al., 2014), based on flux reconstruction. This work adopts FEniCS and its module Dolfin-Adjoint, which are described in this Section.

FEniCS (LOGG, 2007) is open-source software focused on the automated solution of differential equations. Its form compiler (FFC) interprets a discrete form of the weak variational equation, which is written in a high-level language similar to the mathematical syntax, and generates a $\mathrm{C}++$ code for finite element assembly conforming to the Unified Form-Assembly Code (UFC) (ALNÆS et al., 2014). This process relies on the Finite Element Automatic Tabulator (FIAT) (KIRBY, 2004), which numerically builds the finite element basis functions used by FFC. The process is concluded by combining the UFC code with mesh and coefficient data, in order to assemble the FEM tensors. The tensors from the resulting FEM system are given in SciPy format, enabling its solution by several linear algebra packages. However, FEniCS offers native support to PETSc, Epetra, uBLAS and MTL4, allowing to easily specify the desired linear solvers and pre-conditioners. The communication between all FEniCS modules is handled by Dolfin library, providing a unified environment.

\subsubsection{System Decoupling Verification}

It is reported by several authors (SAHOO et al., 2011; MOTA et al., 2004; VASILIEV et al., 2014) that the gradient of pressure observed inside ANG tanks is very small. Therefore, the validity of decoupling the full system presented in Eq. (4.8) is assessed in this Section. Such decoupling is interesting with respect to the topology optimisation implementation, in order to reduce the computational cost.

When decoupling the full adsorption problem in sub-problems, each timestep is formed 
by a sequence of variables updates. The decoupled solution starts with the solution of the continuity equation to obtain the pressure $(P)$ distribution in the domain by keeping the temperature $(T)$ and the adsorbed density $(Q)$ fixed. Based on the new pressure field, the adsorbed density is updated based on the adsorption kinetics, which does not involve spatial derivatives and is explicitly given by pressure and temperature. Finally, the temperature field is obtained by solving the energy balance considering fixed pressure and adsorbed density from the previous step.

The solution of the adsorption problem is then given by

$$
\begin{gathered}
\mathbf{W} \dot{\mathbf{P}}+\mathbf{Z} \mathbf{P}=\mathbf{F}, \\
\mathbf{N} \dot{\mathbf{T}}+\mathbf{L} \mathbf{T}+\mathbf{K} \mathbf{T}=\mathbf{H}
\end{gathered}
$$

whose matrices are assembled based on the coefficients given in Appendix A.3.

The numerical implementation of such system is verified by reproducing the results obtained in Sahoo et al. (2011). The adsorption of Methane on Activated Carbon in an axisymmetric tank for the flow rates of $1 \mathrm{~L} \mathrm{~min}^{-1}, 10 \mathrm{~L} \mathrm{~min}^{-1}$ and $30 \mathrm{~L} \mathrm{~min}^{-1}$ (volume of methane at $300 \mathrm{~K}$ and $\left.P_{a t m}\right)$ is considered. The domain depicted in Figure 4.1 has $L_{o}=0.20 \mathrm{~m}, L_{i}=0.03 \mathrm{~m}, R_{o}=0.05 \mathrm{~m}$ and $R_{i}=0.01 \mathrm{~m}$. The convection heat transfer coefficient on the wall $\Gamma_{W}$ is considered to be $h=5 \mathrm{~W} \mathrm{~m}^{-2} \mathrm{~K}^{-1}$. Due to axisymmetry assumptions, the simulations are based on two-dimensional domains meshed in 43781 triangular finite elements. The material adopted are listed in Appendix B.

The maximum temperature at the center of the tank and the total mass of gas adsorbed for each flow rate case are presented in Figures 4.2-4.4. The plots of the evolution of the central temperature include the datasets of two experiments per each flow rate reported in Sahoo et al. (2011). It can be seen that the numerical implementation of the adsorption problem proposed in this Section approximates satisfactorily the experimental results reported.

Numerical simulations are also reported in Sahoo et al. (2011), which reinforce the accuracy of the results obtained here. The distributions of temperature inside the tanks after each one the flow rates imposed are presented in Figure 4.5. Such distributions present good agreement with the plots reported in the aforementioned work. 


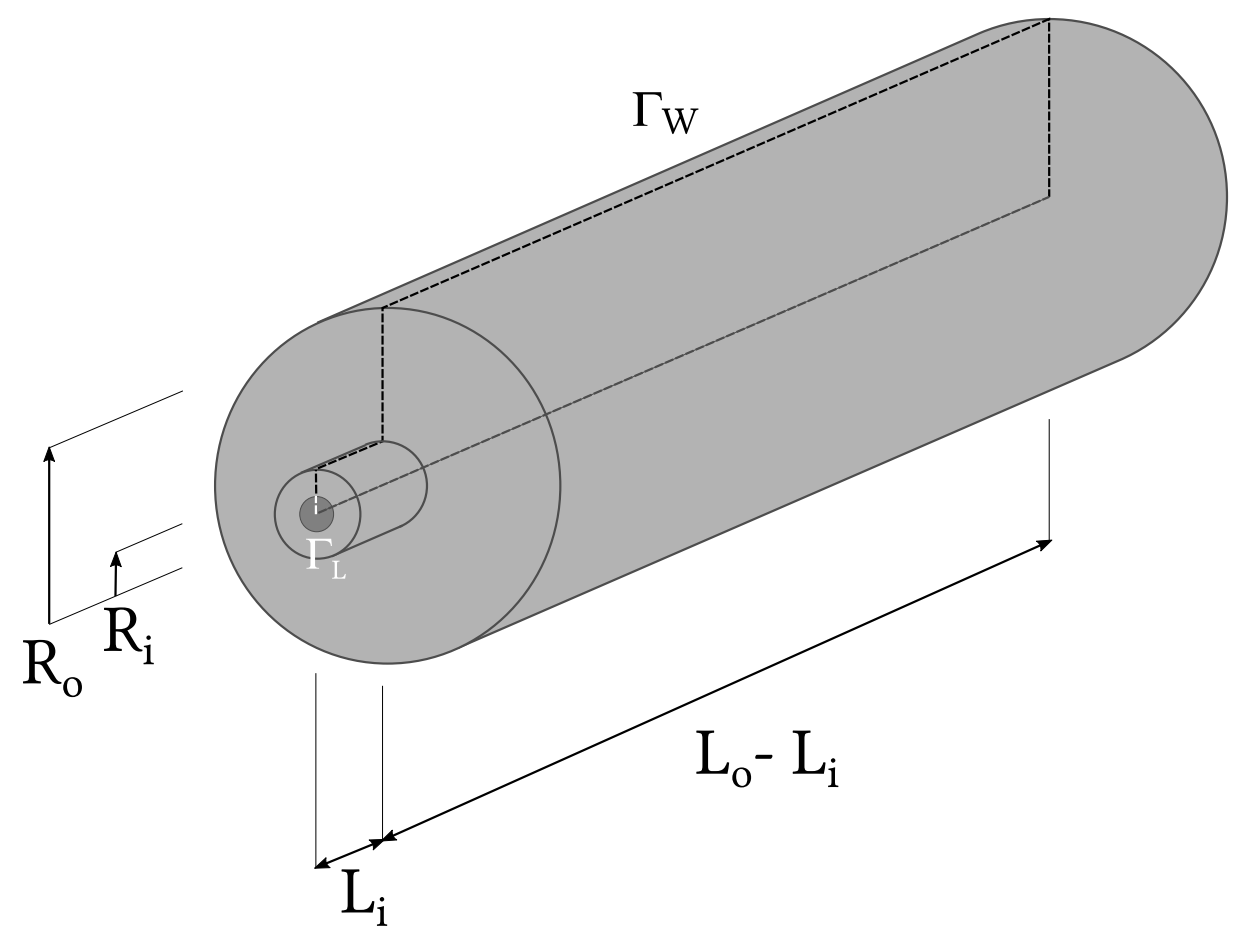

Figure 4.1: Custom axisymmetric tank domain (SAHOO et al., 2011).

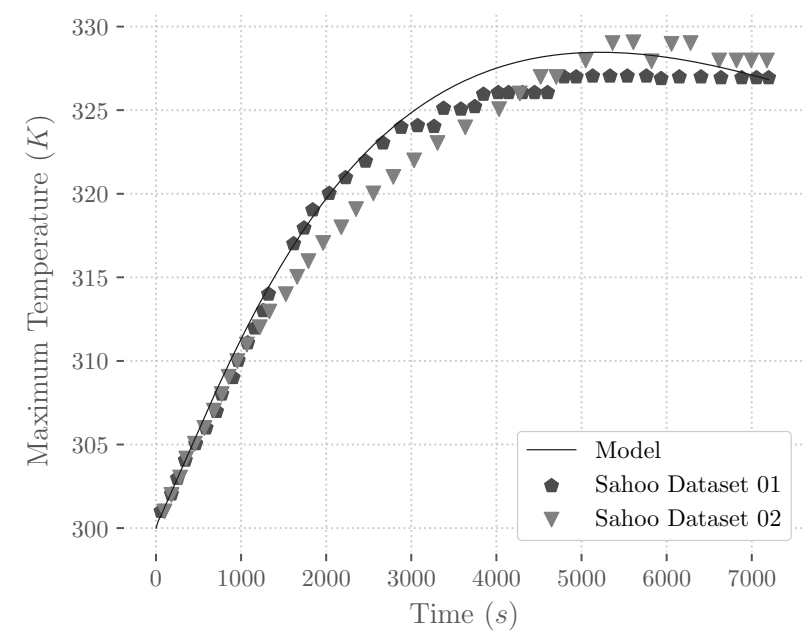

(a) Tank central temperature

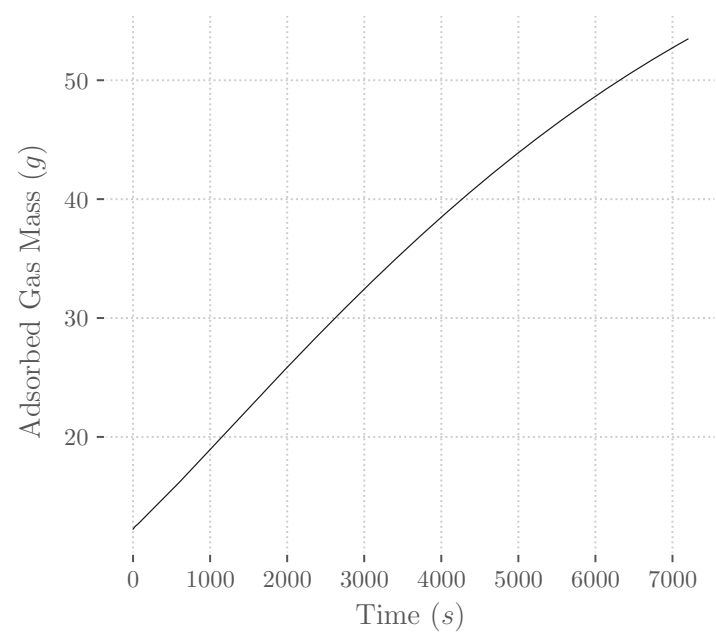

(b) Total adsorbed gas mass

Figure 4.2: Flow rate of $1 \mathrm{~L} \mathrm{~min}^{-1}$ : Comparison with the experimental results in (SAHOO et al., 2011).

\subsubsection{Pressure Loading Verification}

This Section verifies the sensitivity of adsorption cycles to the smoothness factor $\sigma$ defined in Eq. (2.35). Let consider the domain depicted in Figure 4.6, where $R_{i}=$ $0.05 \mathrm{~m}, R_{o}=0.20 \mathrm{~m}, L_{o}=1.00 \mathrm{~m}$, subjected to a smooth pressure increase from $P_{i n i}=$ $0.10 \mathrm{MPa}$ to $P_{\text {let }}=1.00 \mathrm{MPa}$ in a $3600 \mathrm{~s}$ cycle. The temperature at the inlet region $\Gamma_{L}$ 


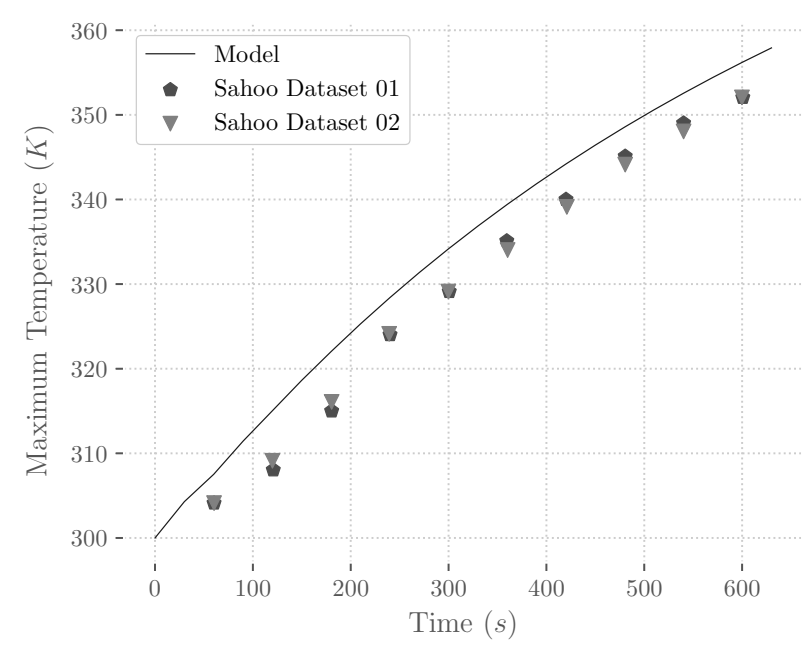

(a) Tank central temperature

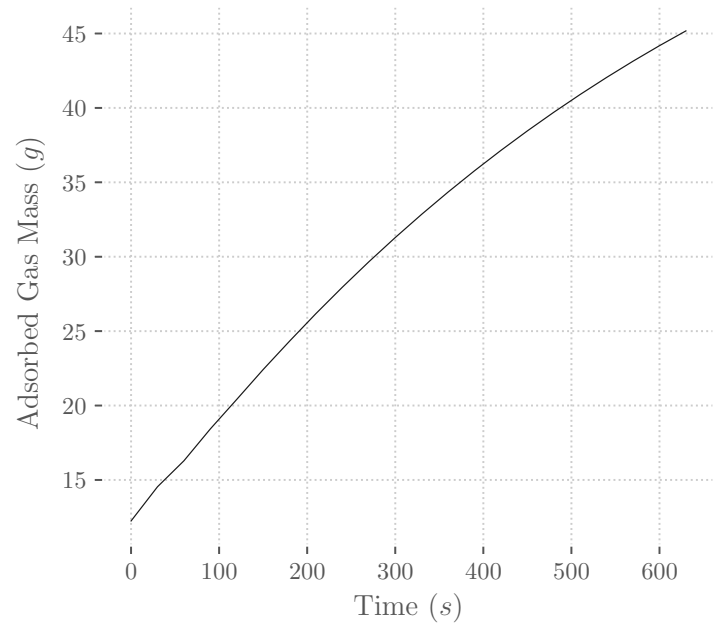

(b) Total adsorbed gas mass

Figure 4.3: Flow rate of $10 \mathrm{~L} \mathrm{~min}^{-1}$ : Comparison with the experimental results in (SAHOO et al., 2011).

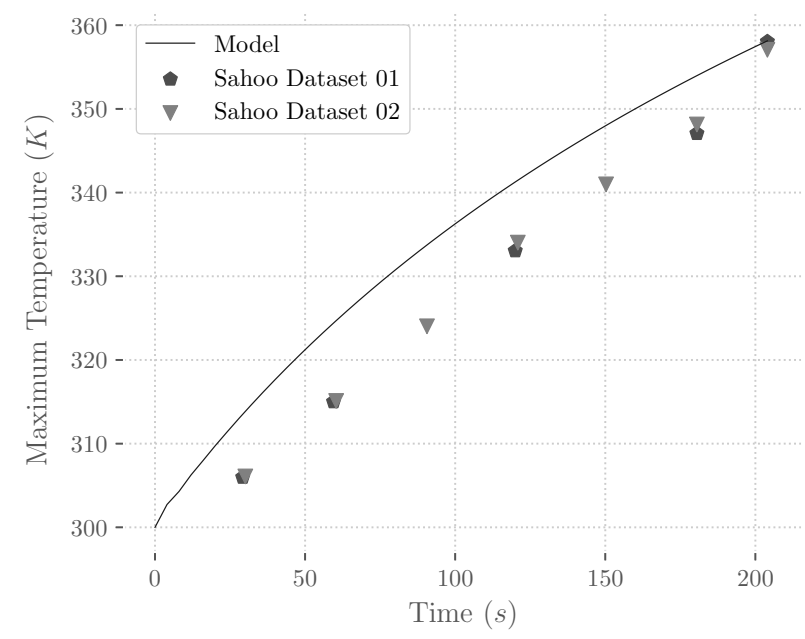

(a) Tank central temperature

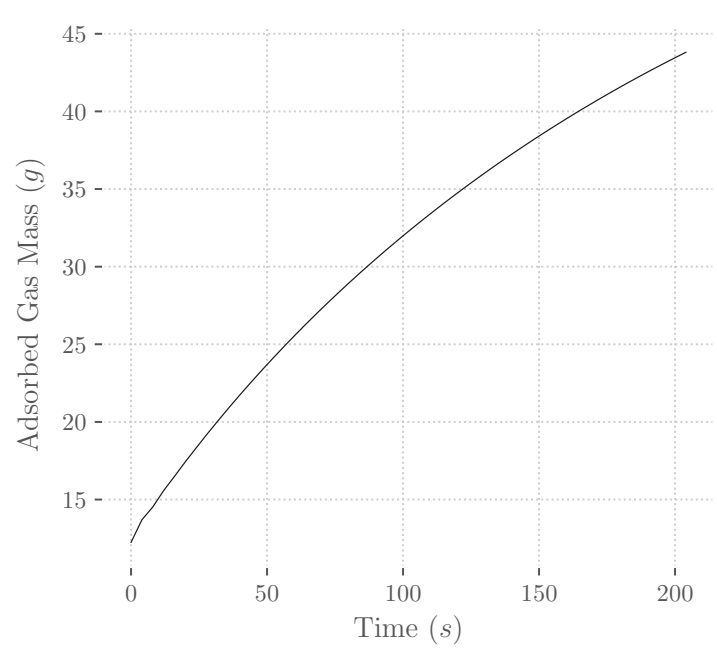

(b) Total adsorbed gas mass

Figure 4.4: Flow rate of $30 \mathrm{~L} \mathrm{~min}^{-1}$ : Comparison with the experimental results in (SAHOO et al., 2011).

during adsorption is kept constant and equal to the entering gas due to the local thermal equilibrium assumption with the gas entering the tank at $293 \mathrm{~K}$.

Figure 4.7 shows the evolution of the total gas mass inside the tank for three different values of $\sigma$. It can be seen that the curves are nearly coincidental after $500 \mathrm{~s}$, demonstrating a low sensitivity to the history of pressure increase at the inlet. As smoothness is decreased, the cycle approaches the case in which the pressure variation is imposed 


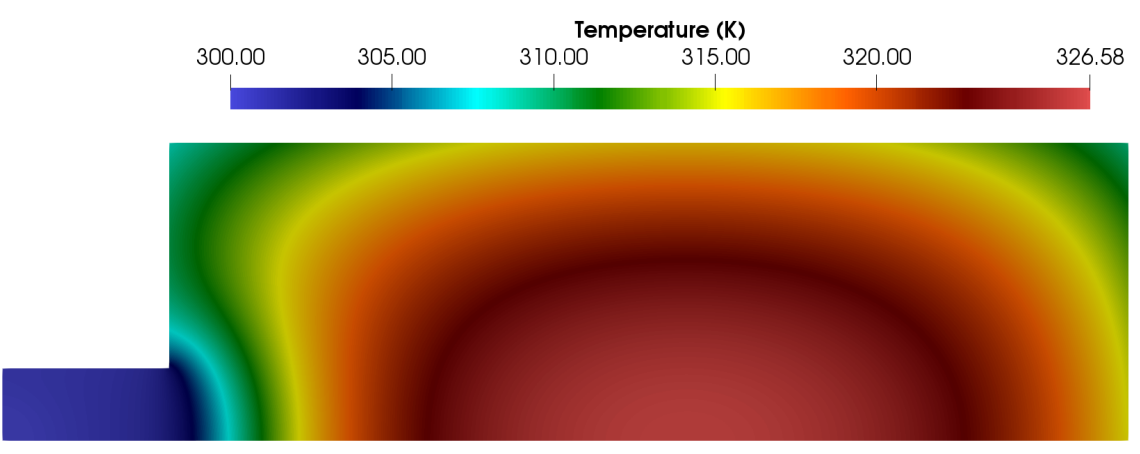

(a) $1 \mathrm{~L} \mathrm{~min}^{-1}$ at $t=7200 \mathrm{~s}$

Temperature $(K)$ $300.00305 .00310 .00315 .00320 .00325 .00330 .00335 .00340 .00345 .00350 .00 \quad 357.84$

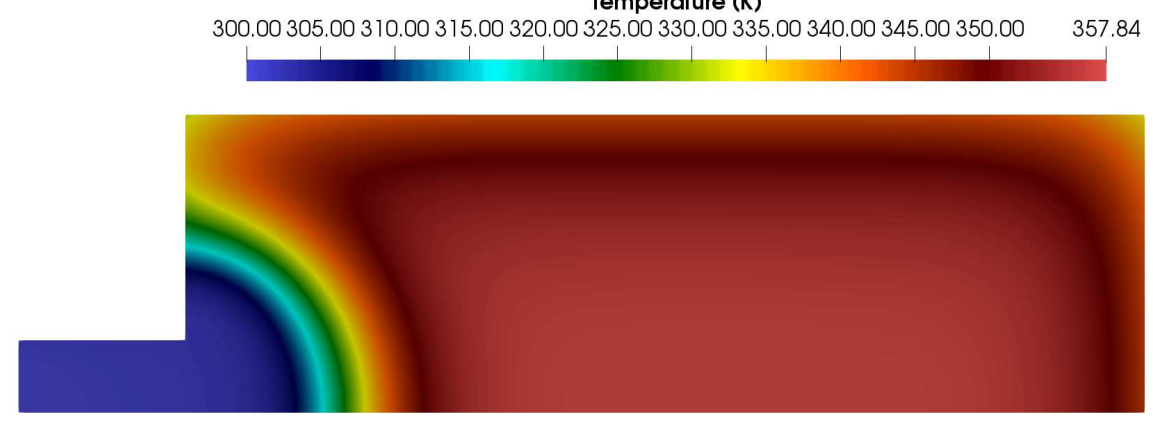

(b) $10 \mathrm{~L} \mathrm{~min}^{-1}$ at $t=630 \mathrm{~s}$

Temperature (K) $300.00305 .00310 .00315 .00320 .00325 .00330 .00335 .00340 .00345 .00350 .00 \quad 357.90$

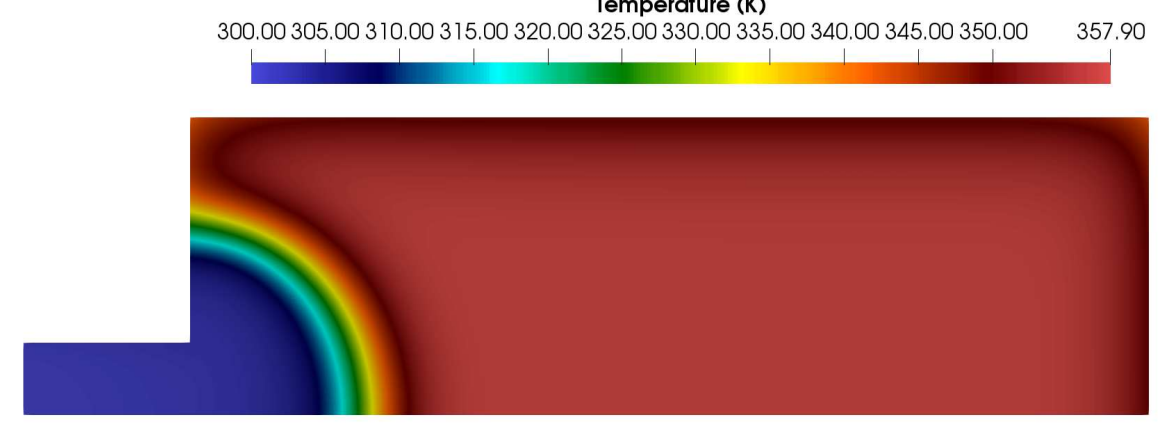

(c) $30 \mathrm{Lmin}^{-1}$ at $t=204 \mathrm{~s}$

Figure 4.5: Temperature distribution at the end of the adsorption cycle.

promply. Figure 4.8 shows how the final total gas mass converges to this case as $\sigma \rightarrow 0$. Therefore, in the scope of this work, it is considered that any $\sigma \leq 0.1\left(\sigma^{-1} \geq 10\right)$ ensures negligible impact to the functional of interest.

\subsubsection{Impermeability Verification}

When distributing a impermeable material, such as Steel, in an ANG tank, it is important to ensure that gas will not flow across it. The impermeability can be numerically represented by assigning very low values to $K_{s}$, given that ideal impermeable media would 


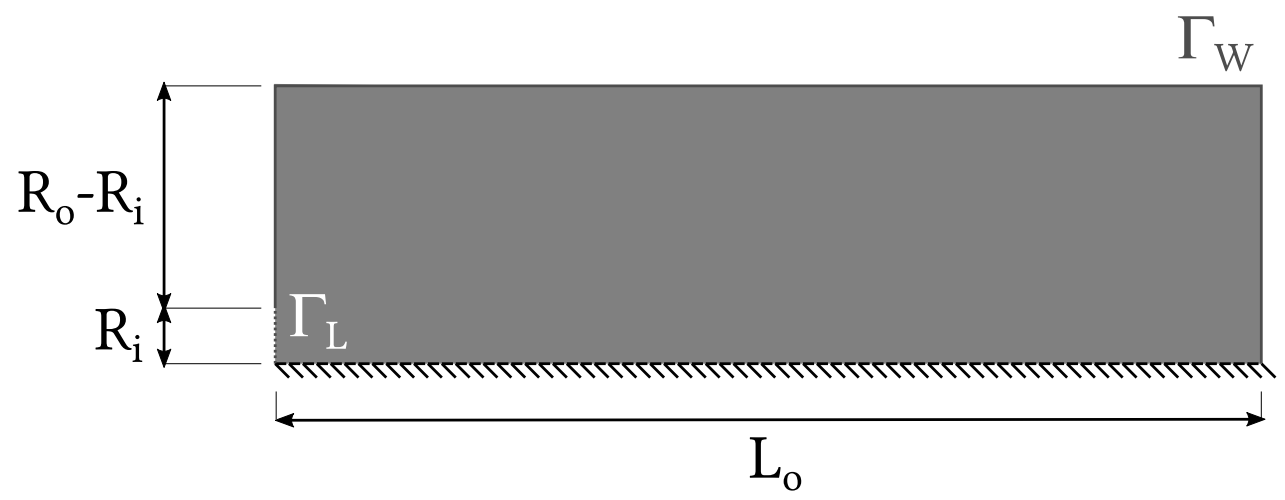

Figure 4.6: Adsorption domain adopted for verifying the sensitivity of adsorption cycles to the smoothness factor $\sigma$.

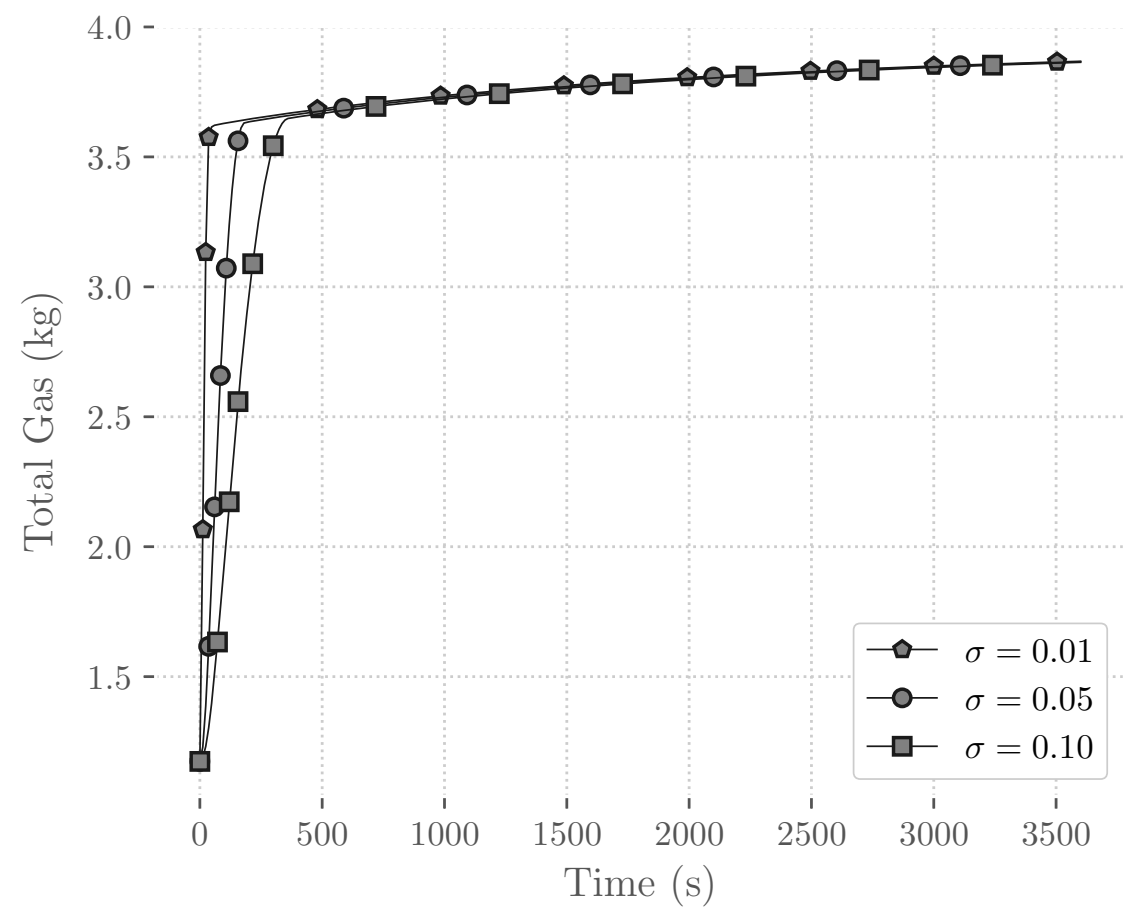

Figure 4.7: Total mass of gas along the adsorption cycle for different smoothness factors.

have $K_{n o n}=0$. Consider the domain in Figure 4.9 representing a section of an axisymmetric tank, constituted of impermeable non-adsorbent and permeable adsorbent phases. The region $\Gamma_{L}$ denotes the inlet region $\left(R_{i}=0.05 \mathrm{~m}\right)$ and $\Gamma_{W}$ denotes walls subjected to heat convection $\left(R_{o}=0.20 \mathrm{~m}\right.$ and $\left.L_{o}=1.00 \mathrm{~m}\right)$. The domain is subjected to a smooth pressure rise on $\Gamma_{L}$ following Eq. (2.35) from $P_{i n i}=0.10 \mathrm{MPa}$ to $P_{\text {let }}$ with $\sigma=0.05$. The permeabilities of $K_{a d s}=3.67 \times 10^{-10} \mathrm{~m}^{2}$ for Activated Carbon and $K_{\text {non }}=1.00 \times 10^{-22} \mathrm{~m}^{2}$ for Steel are considered.

Figure 4.10b demonstrates the inaccessibility of the squared Carbon region on the right to the gas for $P_{l e t}=1.00 \mathrm{MPa}$ in a cycle lasting $t_{f}=600 \mathrm{~s}$. The pressure on the full right 


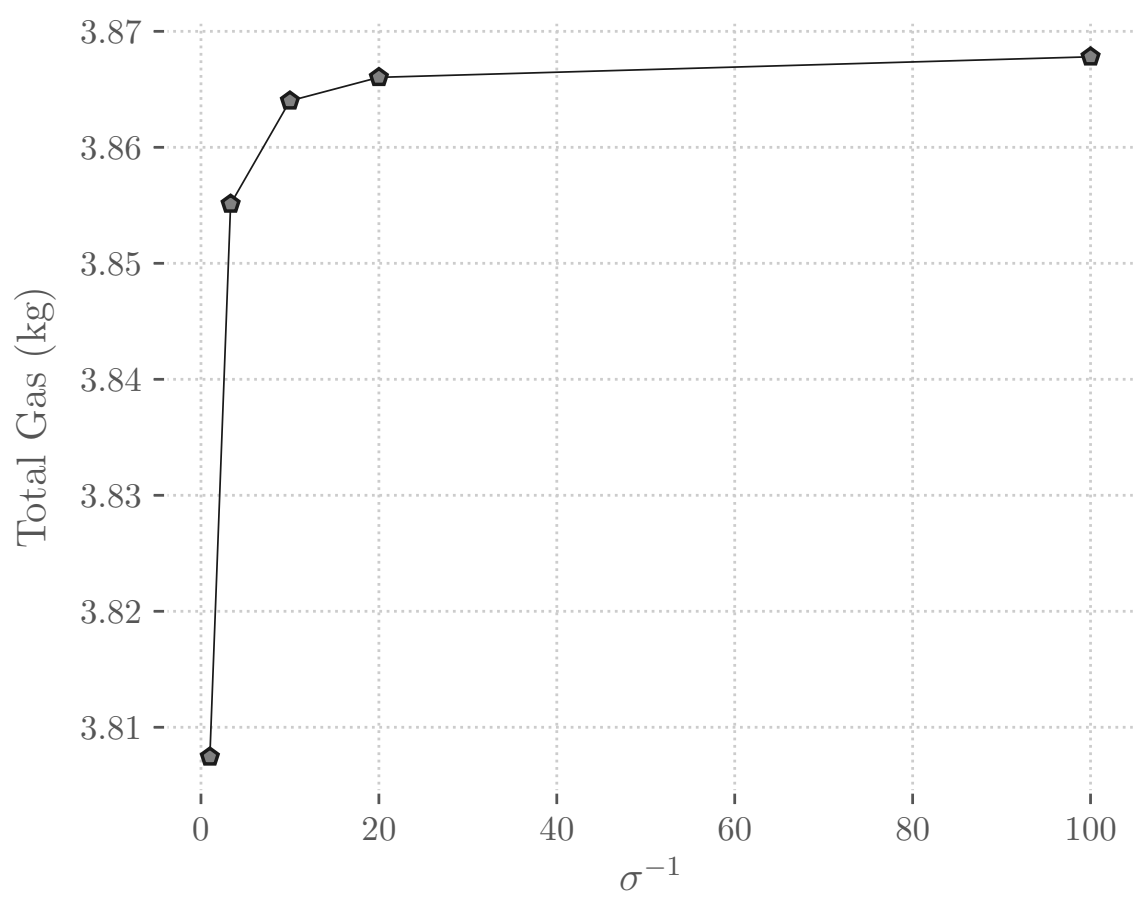

Figure 4.8: Smoothness factor convergence.

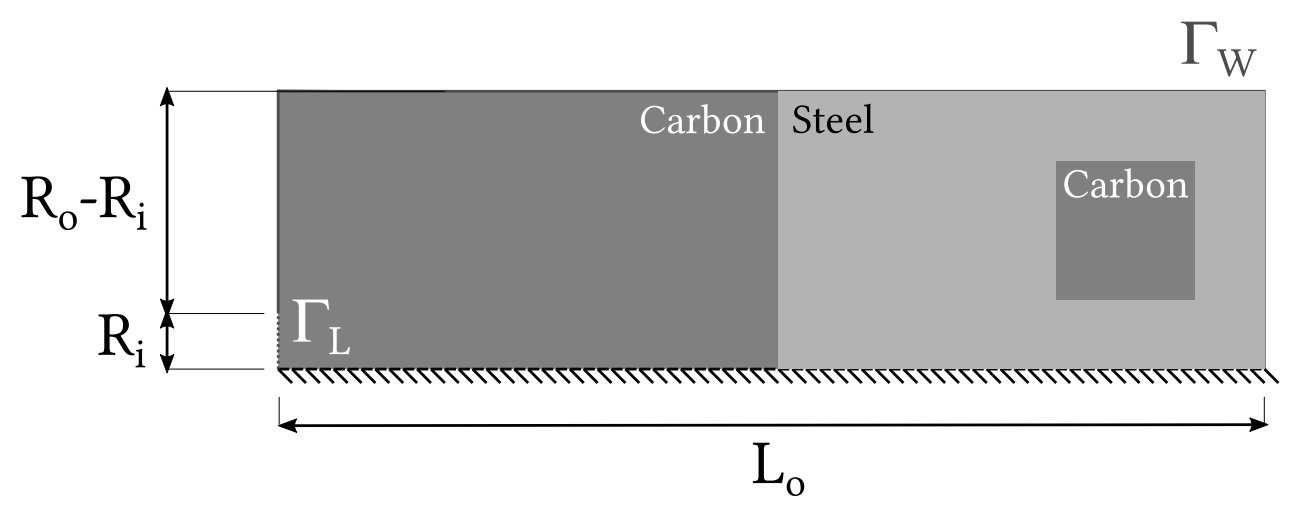

Figure 4.9: Adsorption domain constituted by Activated Carbon and Steel.

half of the domain stays approximately unchanged during the whole cycle, being equal to $P_{i n i}$. The permeability of $1.00 \times 10^{-22}$ has shown to be able to reproduce impermeability even for high pressures and long cycles. In Figure $4.10 \mathrm{c}$ a cycle with $P_{l e t}=8.00 \mathrm{MPa}$ and $t_{f}=3600 \mathrm{~s}$ is considered, demonstrating that that the Carbon region on the right is still not accessed by the gas, although it permeated a slightly larger portion of Steel insulation. 


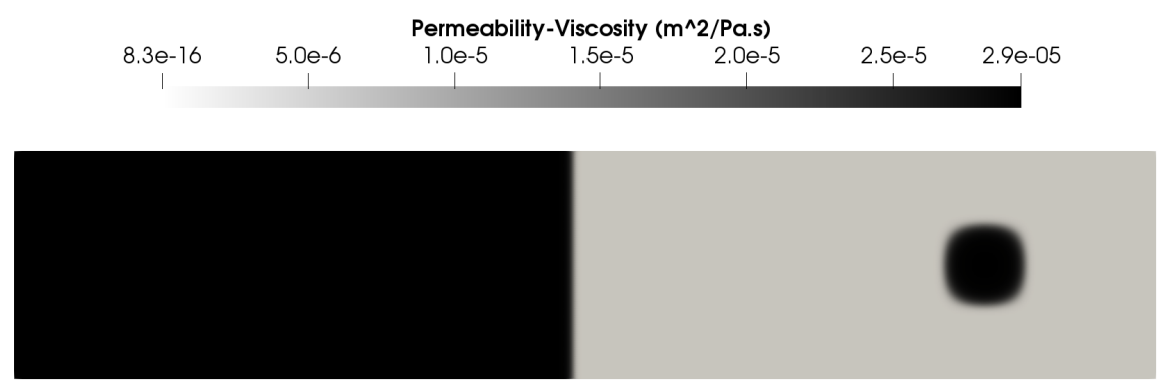

(a) Permeability divided by viscosity $\left(K_{s} \mu_{g}^{-1}\right)$

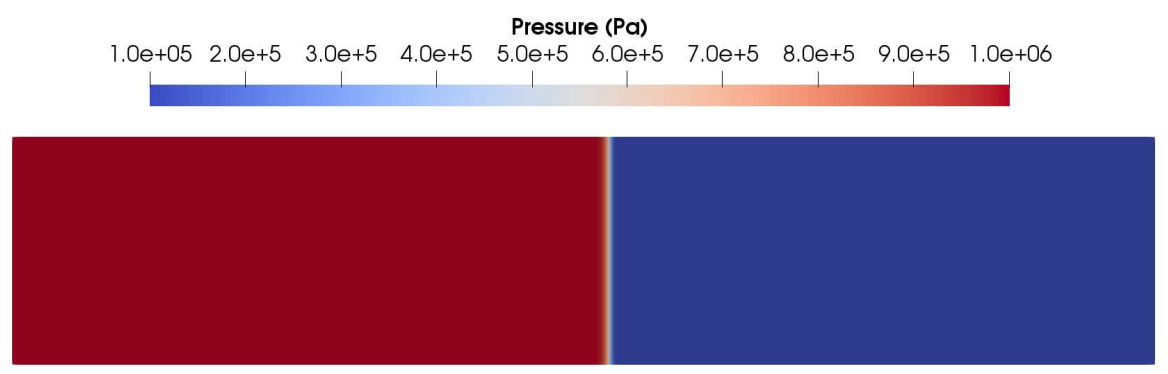

(b) $P_{\text {let }}=1.00 \mathrm{MPa}$ at $t_{f}=600 \mathrm{~s}$

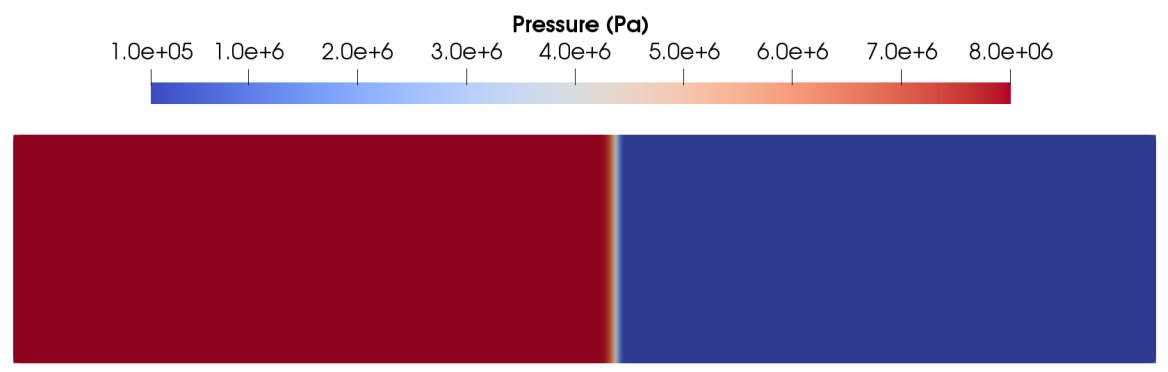

(c) $P_{\text {let }}=8.00 \mathrm{MPa}$ at $t_{f}=3600 \mathrm{~s}$

Figure 4.10: Pressure distribution on Carbon-Steel domain.

\subsubsection{Mesh and Timestep Convergence}

The accuracy of the FEM model is dependent on the mesh and timestep resolutions adopted. The goal of this Section is to identify suitable refinement schemes for the adsorption problems studied here. Let consider the domain depicted in Figure 4.11 subjected to an adsorption cycle following a smooth pressure variation from $P_{i n i}=0.10 \mathrm{MPa}$ to $P_{\text {let }}=1.00 \mathrm{MPa}$ in a $3600 \mathrm{~s}$ cycle with $\sigma=0.1$.

This convergence study is based on the total gas mass intake observed in the adsorption cycle, according to the functional defined in Eq. (3.6). Figure 4.12 presents the variation in the value of such functional for different element sizes, where $\bar{h}^{-1}$ denotes the inverse of the average mesh cell diameter. Very small changes in the functional value are seen for resolutions higher than 50, implying that such value is sufficient for obtaining 


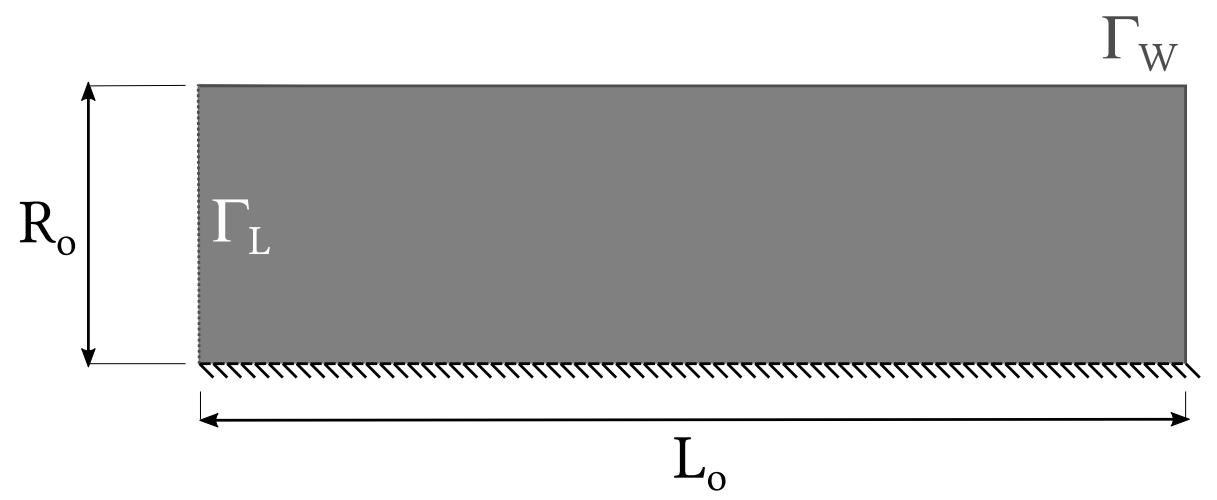

Figure 4.11: Adsorption domain adopted for verifying mesh and timestep convergence.

accurate results. It is important to remark that in topology optimisation problems, higher resolutions can be adopted depending on the desired complexity of the resulting topology.

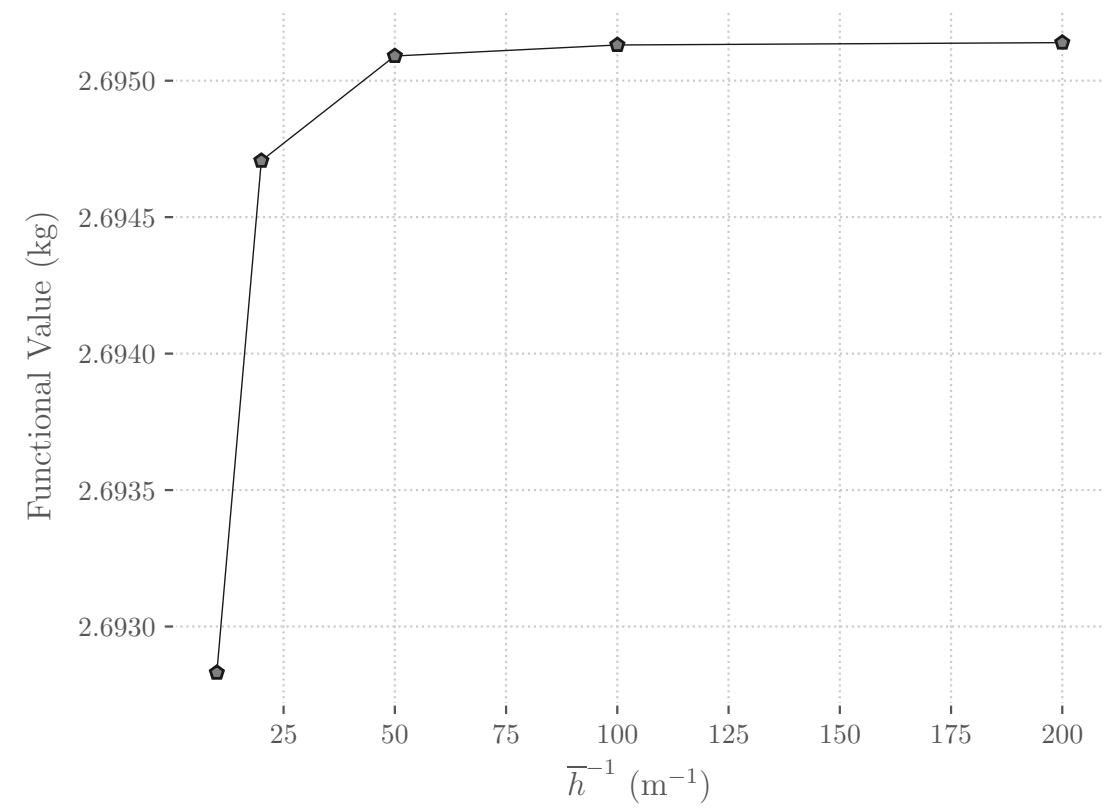

Figure 4.12: Mesh convergence.

Similarly, the timestep adopted in the implicit Euler method for time discretisation can influence the model accuracy. Figure 4.13 presents the total gas mass intake functional for different timesteps $\Delta t$. It can be seen that satisfactory convergence is achieved for $\Delta t=10\left(\Delta t^{-1}=0.1\right)$.

\subsection{Topology Optimisation Implementation}

An important aspect of topology optimisation implementation is the method of obtaining the sensitivities of the functional with respect to the design variables. In this 


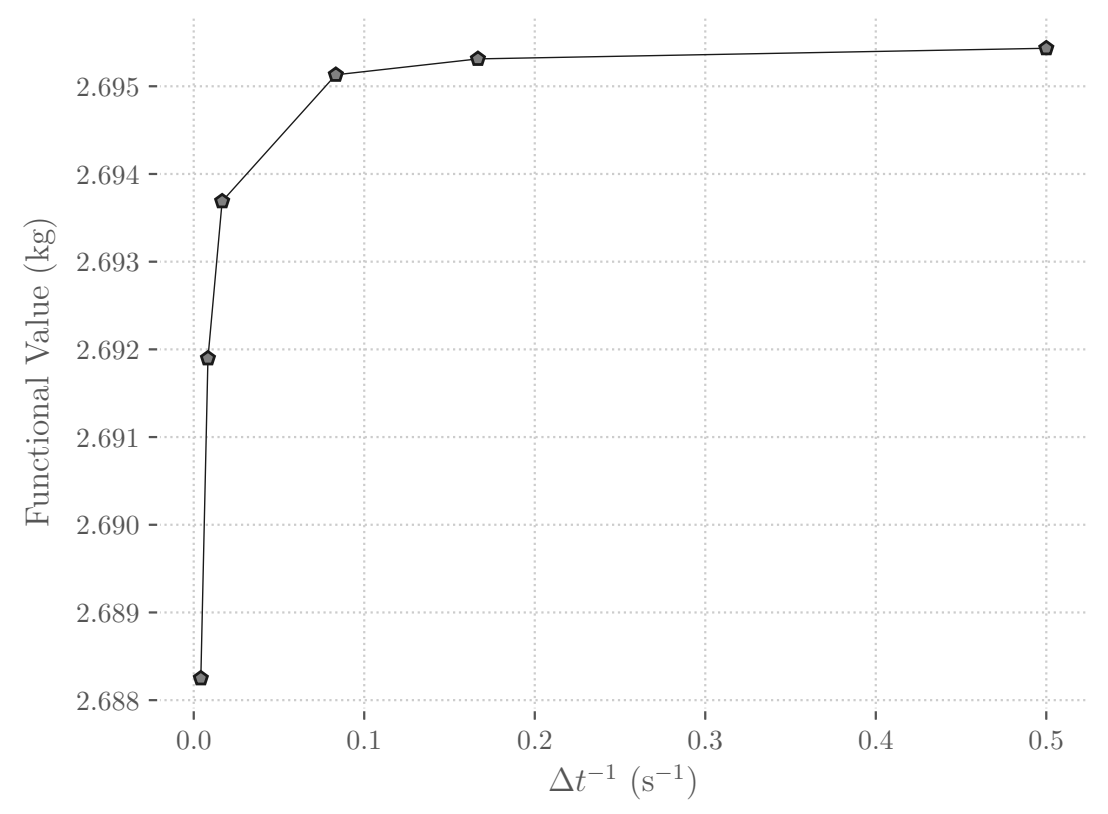

Figure 4.13: Timestep convergence.

work, it is based on the solution of an adjoint problem, which is derived in Section 4.2. Figure 4.14 presents three different approaches for obtaining the adjoint code starting from the continuous forward equations, in the context of the FEniCS packages UFL and UFC. It can be derived by symbolic differentiation of the forward PDE, through packages such as SAGE (STEIN; JOYNER, 2005). The derivation can also be addressed at source code level, treating the forward model as a sequence of elementary instructions by sourceto-source compiler tool for algorithmic differentiation, such as Tapenade (HASCOET; DAUVERGNE, 2008). Another method consists in the derivation of the discrete forward equations, representing a middle-level approach that can be easily automated without depending on low-level implementation details. This work adopts the latter approach aided by the libadjoint library (FUNKE, 2012).

\subsubsection{Design Variables Filtering}

Topology optimisation presents inherent issues such as checker-board instability and mesh dependency, which are usually mitigated by filtering the sensitivities as described in Sigmund \& Petersson (1998). Such method modifies the sensitivity of each design variable based on the values of its neighbours. An alternative approach is to modify the design variables based on the solution of a modified Helmholtz-type PDE, as introduced 


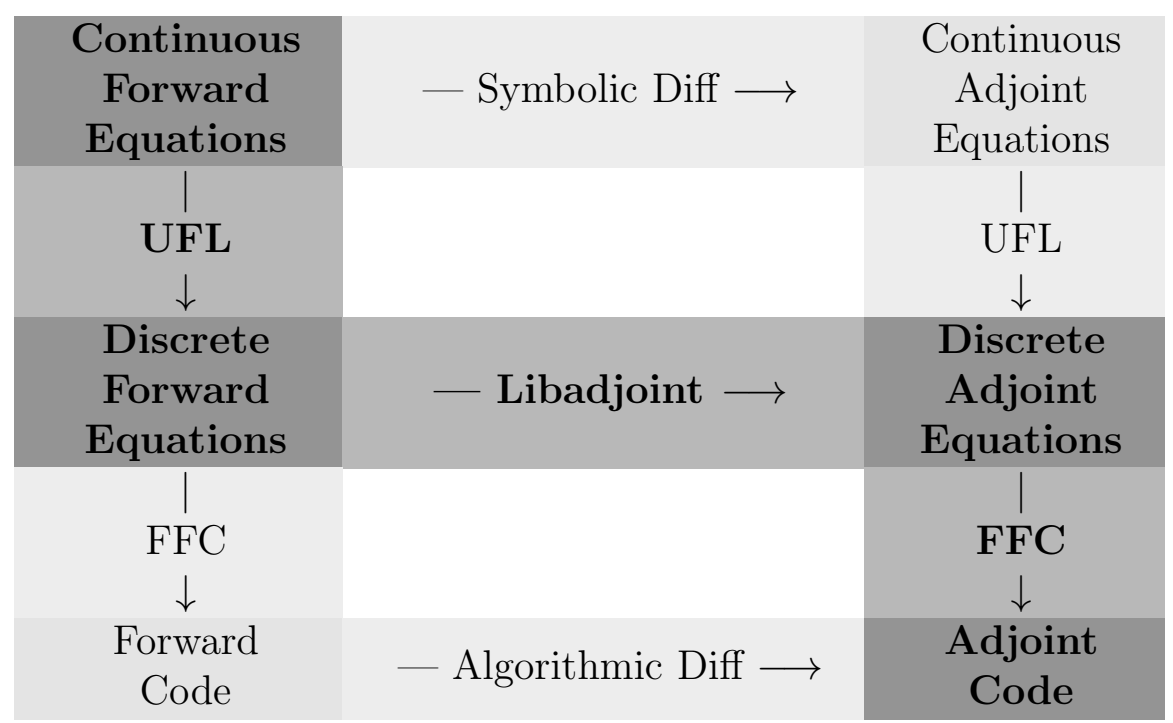

Figure 4.14: Methods for obtaining the adjoint code (FUNKE, 2012).

in Lazarov \& Sigmund (2011), given by

$$
-z^{2} \nabla^{2} \mathrm{e}+\mathrm{e}=\phi
$$

where the distribution $\phi$ is smoothed to e, according to the filter length parameter $z$.

The weak form of the modified Helmholtz-type PDE is given by

$$
\begin{array}{r}
\mathbb{H}=\left\langle\mathrm{e}, v_{H}\right\rangle+\left\langle z^{2} \nabla \mathrm{e}, \nabla v_{H}\right\rangle=\left\langle\phi, v_{H}\right\rangle, \\
\forall v_{H} \in H^{1}(\Omega) .
\end{array}
$$

The discrete filtering system is given by

$$
\mathrm{Ze}=\phi
$$

where $\mathbf{Z}$ and $\boldsymbol{\phi}$ are defined in Appendix A.4.

When applying the Helmholtz filter, despite the functional and PDE systems being expressed in terms of filtered pseudo-densities, $\mathrm{e}_{j}$, the optimisation problem is performed on the field of non-filtered pseudo-densities, $\phi_{j}$, resulting in

$$
\begin{aligned}
\text { Maximise : } & J_{a}(\phi)=\left[\int_{\Omega}\left(Q(\mathrm{e})+\rho_{g}(\mathrm{e})\right) d x\right]_{t_{i n i, a}}^{t_{f i n, a}}, \\
\text { subjected to : } & 0 \leq \phi \leq 1 \\
& \mathbb{M}(P, T, Q)+\mathbb{E}(P, T, Q)+\mathbb{D}(P, T, Q)=0 \\
& -z^{2} \nabla^{2} \mathrm{e}+\mathrm{e}=\phi .
\end{aligned}
$$


Such variable change impacts functionals sensitivities. The implicit derivative of Eq. (4.46) with respect to $\phi_{j}$ results in

$$
\frac{d \mathbf{Z}}{d \phi_{j}} \mathbf{e}+\mathbf{Z} \frac{d \mathbf{e}}{d \phi_{j}}=\frac{d \dot{\phi}}{d \phi_{j}}
$$

where the first term is null, given that $\mathbf{Z}$ is not dependent on pseudo-porosities, and the right-hand side is

$$
\frac{d \dot{\phi}}{d \phi_{j}}=\boldsymbol{\delta}_{j k}
$$

in which $\boldsymbol{\delta}_{j k}$ denotes the Kronecker's delta column vector, being null in all lines, except for the $j$-th, which is equal to 1 .

The sensitivity of the functional $J$ to the non-filtered pseudo-porosity $\phi_{j}$ is then given by

$$
\frac{d J}{d \phi_{j}}=\left(\frac{d J}{d \mathbf{e}}\right)^{T} \frac{d \mathbf{e}}{d \phi_{j}}=\left(\frac{d J}{d \mathbf{e}}\right)^{T} \mathbf{Z}^{-1} \boldsymbol{\delta}_{j k} .
$$

\subsubsection{Optimisation Algorithm}

The optimisation algorithm follows the flow represented in Figure 4.15. In the optimisation loop, the sensitivities are obtained via Dolfin-Adjoint (FUNKE, 2012) based on the equilibrium equations solved on FEniCS. An optimiser receives the sensitivities and output a new distribution of design variables, aiming to improve the functional of interest. The relative functional variation is defined by considering the last two iterations by

$$
\omega=\frac{J_{i t}-\frac{J_{i t-1}+J_{i t-2}}{2}}{\frac{J_{i t-1}+J_{i t-2}}{2}} .
$$

When the functional relative variation is non-negative and smaller than a tolerance $\omega_{\text {min }}$ or when the maximum number of iterations $i t_{\text {max }}$ is reached, the loop is broken. The penalisation $p$ is then increased by $\Delta p$, following the continuation method, and the loop is restarted. This algorithm is performed until reaching the maximum penalisation $p_{\max }$, when the final topology is defined.

The optimiser adopted in all the cases presented in this work is the limited memory Broyden-Fletcher-Goldfarb-Shanno optimiser with box constraints (L-BFGS-B) (BYRD et al., 1995) implemented in scipy.optimize package (JONES et al., 2011). Optimisation problems requiring volume constraints can be approached based on other optimisers contained in this package, such as SLSQP and COBYLA, or via an IPOpt (WACHTER; 


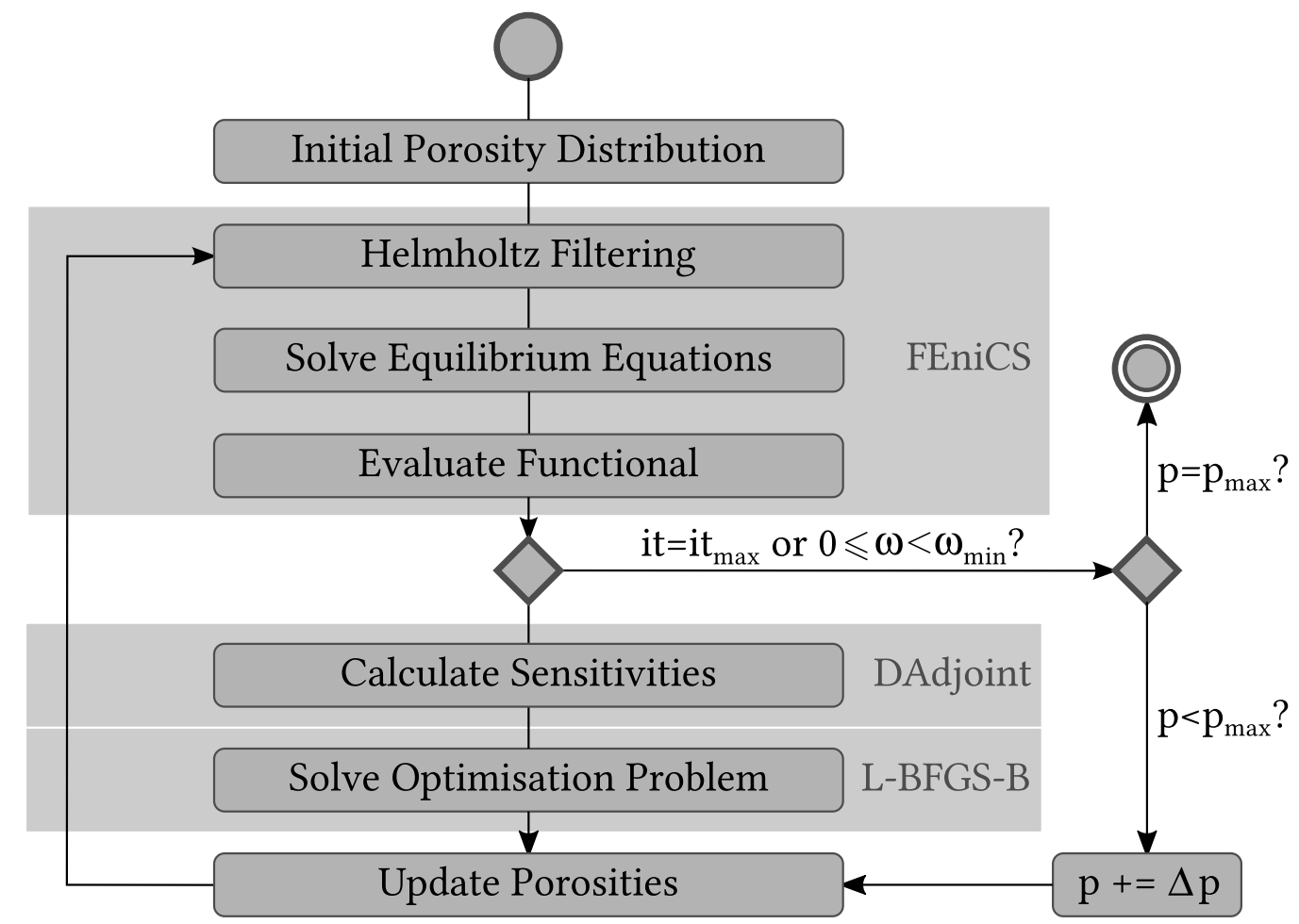

Figure 4.15: Optimisation activity diagram.

BIEGLER, 2006) interface also available in Dolfin-Adjoint.

\subsubsection{Adjoint Sensitivites Verification}

The topology optimisation relies on the accurate computation of the sensitivity of the objective functions to the design variables. The gradients computed based on the adjoint problem described in Section 4.2 can be verified through a Taylor remainder convergence test. Given a reduced functional $\hat{J}(m)$ subjected to an arbitrary perturbation $\delta m$, the first-order Taylor remainder tends to zero at $\mathcal{O}(h)$ while the second-order tends at $\mathcal{O}\left(h^{2}\right)$. That means that by halving $h$ the first-order error also halves while the second-order error is divided by four. Taylor remainders expressions are given in Eq. (4.52). By evaluating the second-order remainder based on the $\nabla \hat{J}$ obtained via adjoint approach, its correctness can be determined by observing $\mathcal{O}\left(h^{2}\right)$ convergence.

$$
\begin{aligned}
|\hat{J}(m+h \delta m)-\hat{J}(m)| \rightarrow 0 & \text { at } \mathcal{O}(h), \\
|\hat{J}(m+h \delta m)-\hat{J}(m)-h \nabla \hat{J} \cdot \delta m| \rightarrow 0 & \text { at } \mathcal{O}\left(h^{2}\right) .
\end{aligned}
$$

Due to current Dolfin-Adjoint limitations, varying Dirichlet boundary conditions will produce inaccurate gradients. Thus, the variation of the inlet pressure on $\Gamma_{L}$ is done 
through the Nitsche's method (JUNTUNEN; STENBERG, 2012) by

$$
\frac{\partial P}{\partial n}=\frac{1}{\delta}\left(P_{l e t}-P\right) \quad \text { on } \Gamma_{L}
$$

in which $P \rightarrow P_{l e t}$ as $\delta \rightarrow 0$.

Such condition is imposed to the system by enforcing

$$
\left\langle\frac{M_{g} K_{s}}{R_{g} \mu_{g}} \frac{P}{T} \nabla P, v_{M}\right\rangle_{\Gamma_{N}}=\left\langle\frac{M_{g} K_{s}}{R_{g} \mu_{g}} \frac{P}{T} \frac{1}{\delta}\left(P_{l e t}-P\right), v_{M}\right\rangle_{\Gamma_{L}} .
$$

Let consider the same cycle adopted in Section 4.3.2, with $\sigma=0.1$, and the functional of total gas mass intake along such cycle, given by Eq. (3.6). From an initial homogeneous distribution of design variables $\mathbf{e}=\mathbf{0 . 5}$ the domain is subjected to a pseudo-random perturbation $\delta e$ scaled to different values of $h$ as shown in Figure 4.16.

Figure 4.16: Original (top) and Perturbed Pseudo-Porosity fields for (from the top) $h=1.00 \times 10^{-3}, h=5.00 \times 10^{-4}, h=2.50 \times 10^{-5}, h=1.25 \times 10^{-5}, h=6.25 \times 10^{-6}$.
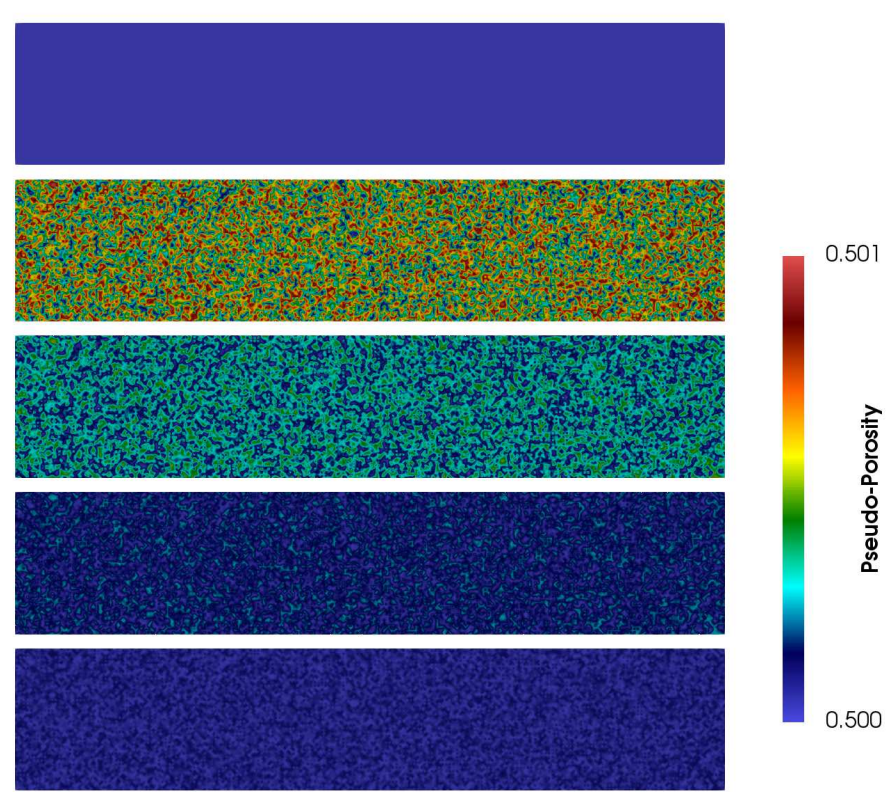

0.500

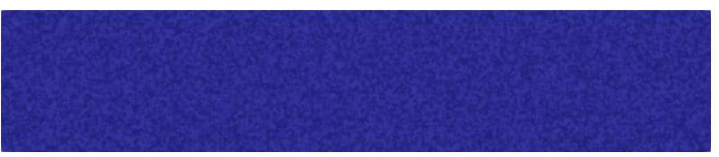

Table 4.1 presents the errors for each perturbation following the first and second Taylor remainder expressions. It demonstrates the correctness of $\nabla \hat{J}$ as the convergence order of second-order Taylor remainders are approximately 2.0.

Such verification of the sensitivities, associated with the afore presented verifications concerning the adsorption problem, sustains the numerical implementation in all its aspects. In the following Chapter, optimisation problems are approached based on the 
Table 4.1: Taylor Remainder Convergence.

\begin{tabular}{lcc}
\hline$h$ & Taylor Remainder & Convergence Order \\
\hline 1st Order $1.00 \times 10^{-3}$ & $1.47 \times 10^{-3}$ & \\
1st Order $5.00 \times 10^{-4}$ & $7.36 \times 10^{-4}$ & 0.99929 \\
1st Order $2.50 \times 10^{-5}$ & $3.68 \times 10^{-4}$ & 0.99964 \\
1st Order $1.25 \times 10^{-5}$ & $1.84 \times 10^{-4}$ & 0.99982 \\
1st Order $6.25 \times 10^{-6}$ & $9.21 \times 10^{-5}$ & 0.99991 \\
\hline 2nd Order $1.00 \times 10^{-3}$ & $1.43 \times 10^{-6}$ & \\
2nd Order $5.00 \times 10^{-4}$ & $3.58 \times 10^{-7}$ & 1.99989 \\
2nd Order $2.50 \times 10^{-5}$ & $8.94 \times 10^{-8}$ & 1.99996 \\
2nd Order $1.25 \times 10^{-5}$ & $2.23 \times 10^{-8}$ & 2.00007 \\
2nd Order $6.25 \times 10^{-6}$ & $5.59 \times 10^{-9}$ & 2.00013 \\
\hline
\end{tabular}

method derived here, resulting in several optimised adsorbent bed topologies. 


\section{Results and Discussion}

This Chapter starts by comparing the OHT and traditional material models. Thereafter, the topology optimisation method derived in this work is adopted for designing ANG tanks for on-demand consumption of fuel.

\subsection{Definition of Standard Domains and Cycles for the Design of ANG Tanks}

The following Sections are focused on the design of ANG tanks for on-demand fuel consumption based on the idea presented in Section 3.1, in which which non-adsorbent materials are distributed in the adsorbent bed. The objective function adopted is given by the total mass intake of gas during adsorption cycles of pre-defined length, as presented in Section 3.3. The major drawback when designing these tanks is the exothermic nature of the adsorption reaction, which increases the adsorbent bed temperature and hinders reaching of the equilibrium density of adsorbed gas. Such behaviour poses several limitations to the range of applicability of ANG tanks and asks for some thermal management, which can be done passively or actively. A passive method is based merely on the heat transfer with the environment, without the addition of any external work for cooling. In the case of aiding the tank filling by the imposition of forced convection, cold walls or any other sort of cooling method which requires additional work, the thermal management is considered to be active. In the latter, exergy analyses must be done in order to quantify how efficient is the system as a whole, although such analyses are beyond the scope of this work.

The domains considered here represent tanks constituted by adsorbent beds wrapped by a thin sheet of metallic material subject to heat exchange through convection on its walls, denoted by $\Gamma_{W}$. The contribution of such wall is assumed to be negligible (SAHOO 
et al., 2011) and it is not included in the model. In fact, being a thin metallic sheet, its Biot number $(\mathrm{Bi})$ is very small and the heat conduction inside it is much faster than the heat convection away from the surface.

The domains studied here represent two-dimensional reductions of three-dimensional tanks. The first domain, presented in Table 5.1, consists in an approximation of a very long prismatic tank with fixed cross-section. Since ANG tanks operate in a much lower pressure than its CNG pairs, prismatic tanks can offer a good trade-off between stress concentration and packing density or efficiency in space usage. Based on symmetry, only one quarter of such section is optimised. The gas inlet is considered to be distant from such section, making the pressure homogeneous on the whole domain and, consequently, the convective term in the energy balance null.

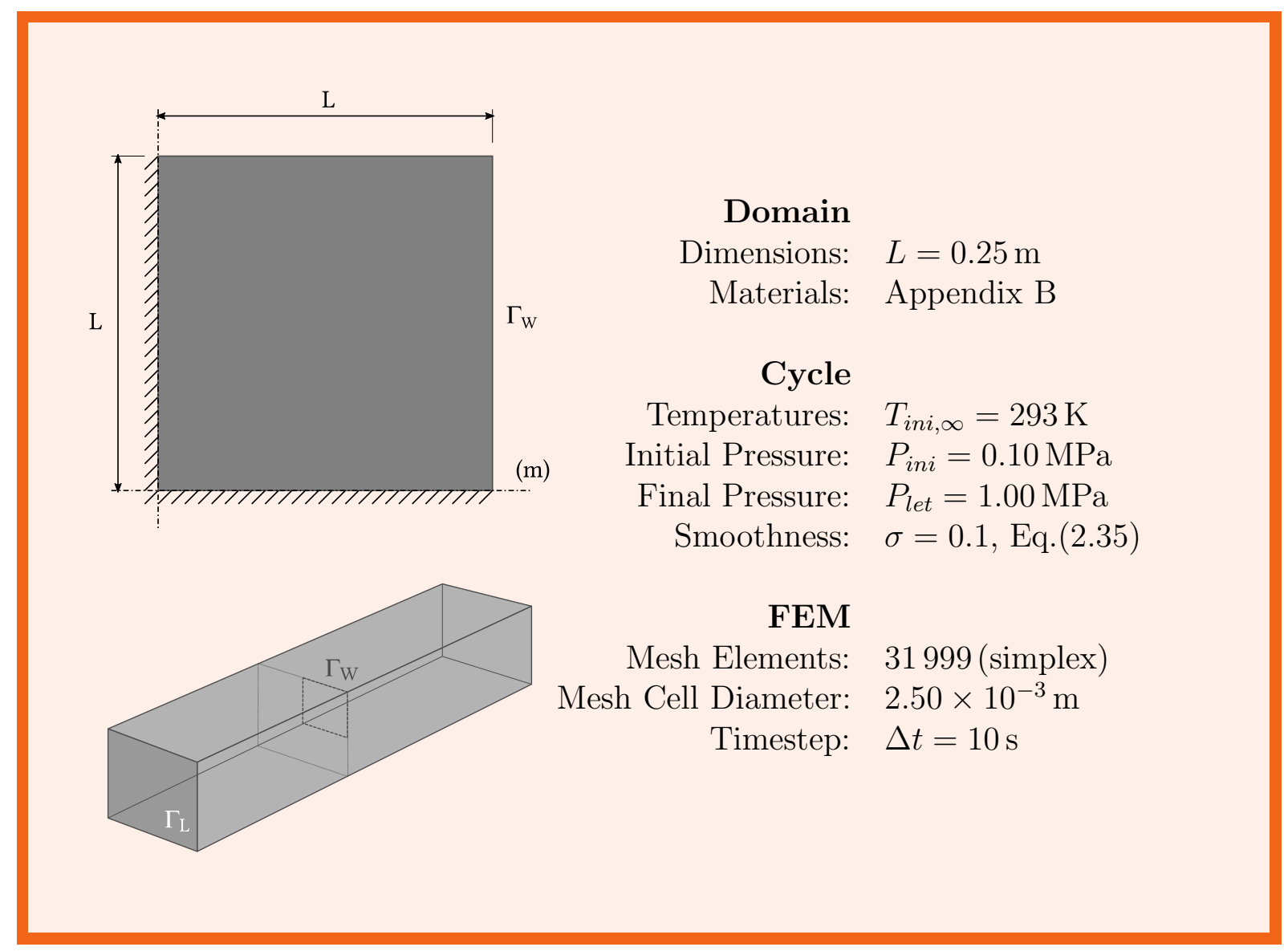

Table 5.1: Standard domain and cycle for prismatic tanks.

The second domain is presented in Table 5.2, consisting of an axisymmetric tank represented by its radial section. In this case, pressure gradient is observed across the domain, originated by the gas inlet region denoted by $\Gamma_{L}$. In some of the optimisation studies presented in this Chapter, low temperature is imposed on the region denoted by 
$\Gamma_{F}$

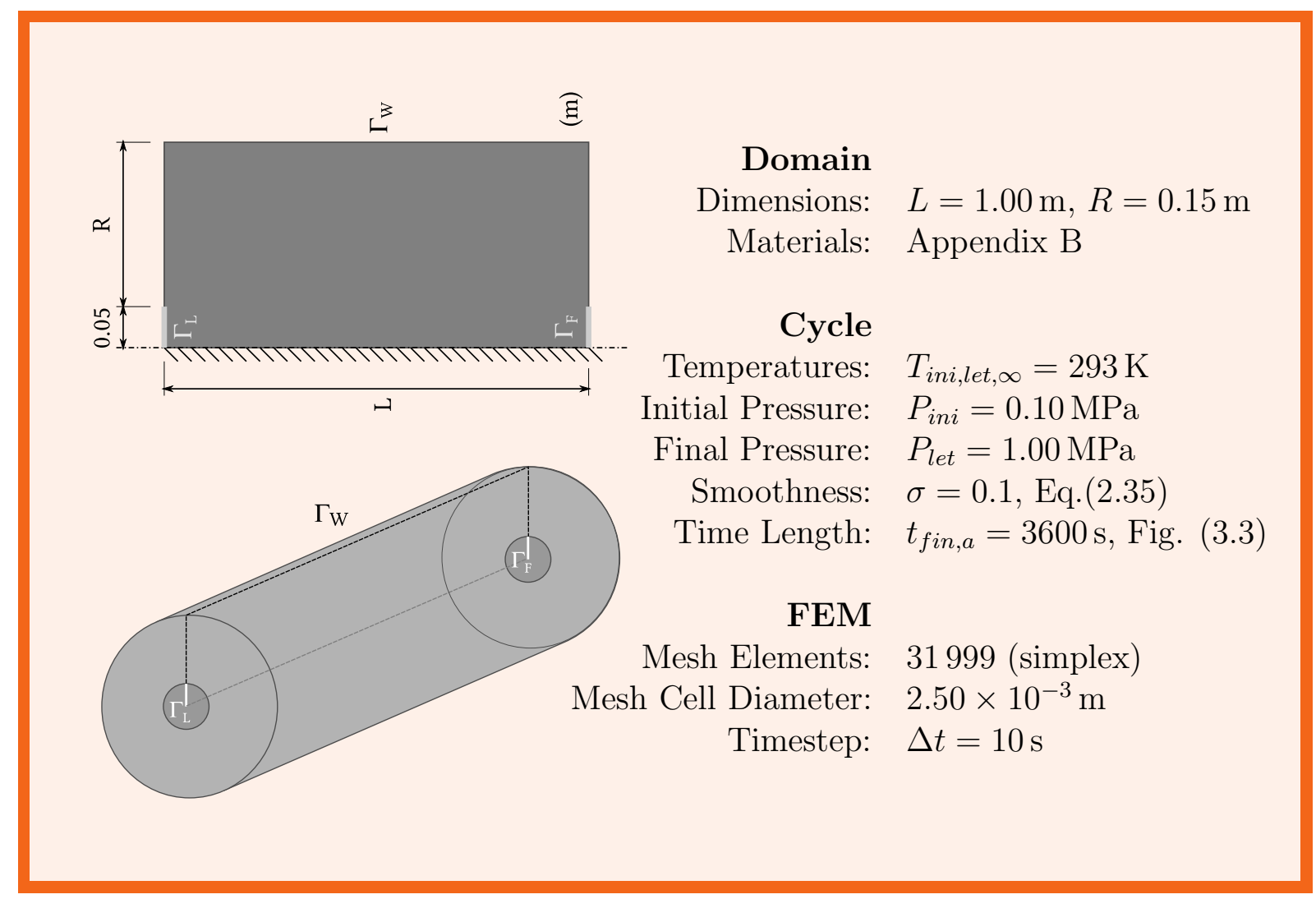

Table 5.2: Standard domain and cycle for cylindrical tanks.

The third and last domain adopted in this work is presented in Table 5.3. It follows the axisymmetry assumption but is focused on assessing the influence of complex shapes on the optimisation of ANG tanks beds. Despite not modelling any specific scenario, such domain presents typical features observed in tanks fitted along other components in constrained spaces. Similar to the previous domain, gas inlet occurs on the region $\Gamma_{L}$ and cold temperature is imposed on $\Gamma_{F}$ in some of the optimisation cases studied. As it will be seen in the sequence of this Chapter, concave and convex corners play an important role in the behaviour of ANG tanks, being, therefore, essential for the scope of this work. 


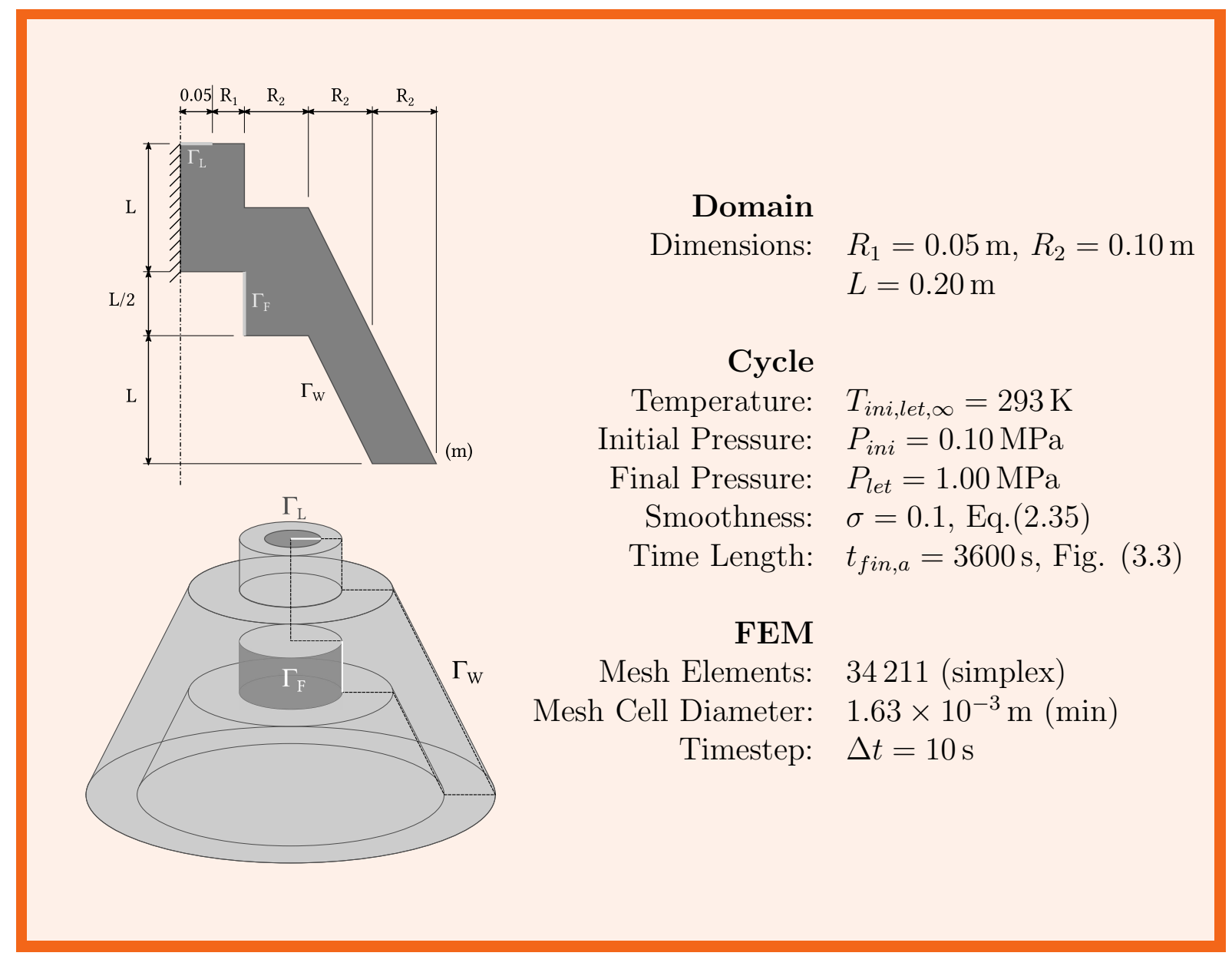

Table 5.3: Standard domain and cycle for a custom axisymmetric tank. 


\subsection{Evaluation of the OHT Material Model for the Design of ANG Tanks}

This Section is focused on studying the properties of the OHT material model. It is initially compared with SIMP and then tested for the dependency on the initial guess and penalisation continuation when adopted in the design of ANG tanks.

\subsubsection{OHT vs. Traditional Material Models}

The OHT material model proposed in Section 3.4.2 aims to mitigate the theoretical advantage of intermediate materials arising from the relaxation of the optimisation problem. This Section compares OHT with the traditional SIMP, emphasazing the relevance of the offset imposed to the model.

The optimisation of a prismatic tank, defined in Table 5.4 is chosen to test different material models. Figure 5.1 shows the evolution of the distribution of the design variables for three different material models. The first column is based on SIMP, in which the penalisation was continuated from $p=1$ to $p=5$, with $\Delta p=1$. The central column shows the evolution for a material model based on hyperbolic tangents without an offset, expressed by the Eq. (3.13) with $\epsilon=0.0$, in which the penalisation is continued from $p=1$ to $p=51$, with $\Delta p=5$. The right column shows the evolution of the same problem when an offset is defined by imposing $\epsilon=0.02$. It is important to note that the penalisation coefficients in SIMP and OHT material models do not present a clear correlation, in a way that $p=51$ in OHT does not represent, necessarily, a more penalised material model than $p=5$ in SIMP.

It can be seen that the adoption of SIMP causes the pseudo-porosity values to be near $\mathrm{e}=0.92$, given that such value offers a reasonable increase of the thermal conductivity for an adsorption capacity near the full-carbon phase. Even when very high penalisation is imposed to the material model, such anomaly will still occur around the phase transition region. If such region is translated from $\mathrm{e}=1.0$ to $\mathrm{e}=0.5$ by adopting an hyperbolic tangent material model, the resulting evolution of the optimisation will be similar to the central column in Figure 5.1. Again, the values of the design variables are all concentrated around the phase transition region, taking advantage from the desired properties from each phase. In this optimisation problem, the offset tangents material model, presented in the third column, is the only one able to avoid unfeasible intermediate properties and define a clear topology. In this last case, as the penalisation increases the intermediate 
pseudo-porosity of $\mathrm{e}=0.5$ becomes increasingly less advantageous for the functional of interest.

Figure 5.2 complements the points aforementioned by presenting the convergence of each one of the optimisations performed. Each drop seen in the curves are due to the increase of the penalisation, following the continuation scheme adopted. In SIMP and nonoffset hyperbolic tangents material models, a theoretical maximum based on unfeasible materials is carried along the iterations, unaffected by the penalisation increase. In the case of OHT, the functional value decreases along the iterations as it recovers the binary nature of the problem as the penalisation is increased.

\section{Domain \\ Heat Convection: $\quad h_{\Gamma_{W}}=100 \mathrm{Wm}^{-2} \mathrm{~K}^{-1}$}

Prismatic Tank

(Table 5.1)

\section{Cycle}

Time Length: $\quad t_{f i n, a}=10800 \mathrm{~s}$, Fig. (3.3)

\section{Optimisation}

Iterations per Step: $i t_{\max }=20$

Tolerance: $\quad \omega_{\min }=1.0 \times 10-6$

Filter Radius: $z=5 \times 10^{-3} \mathrm{~m}$, Eq.(4.44)

Table 5.4: Optimisation definitions for a prismatic tank subjected to forced convection. 

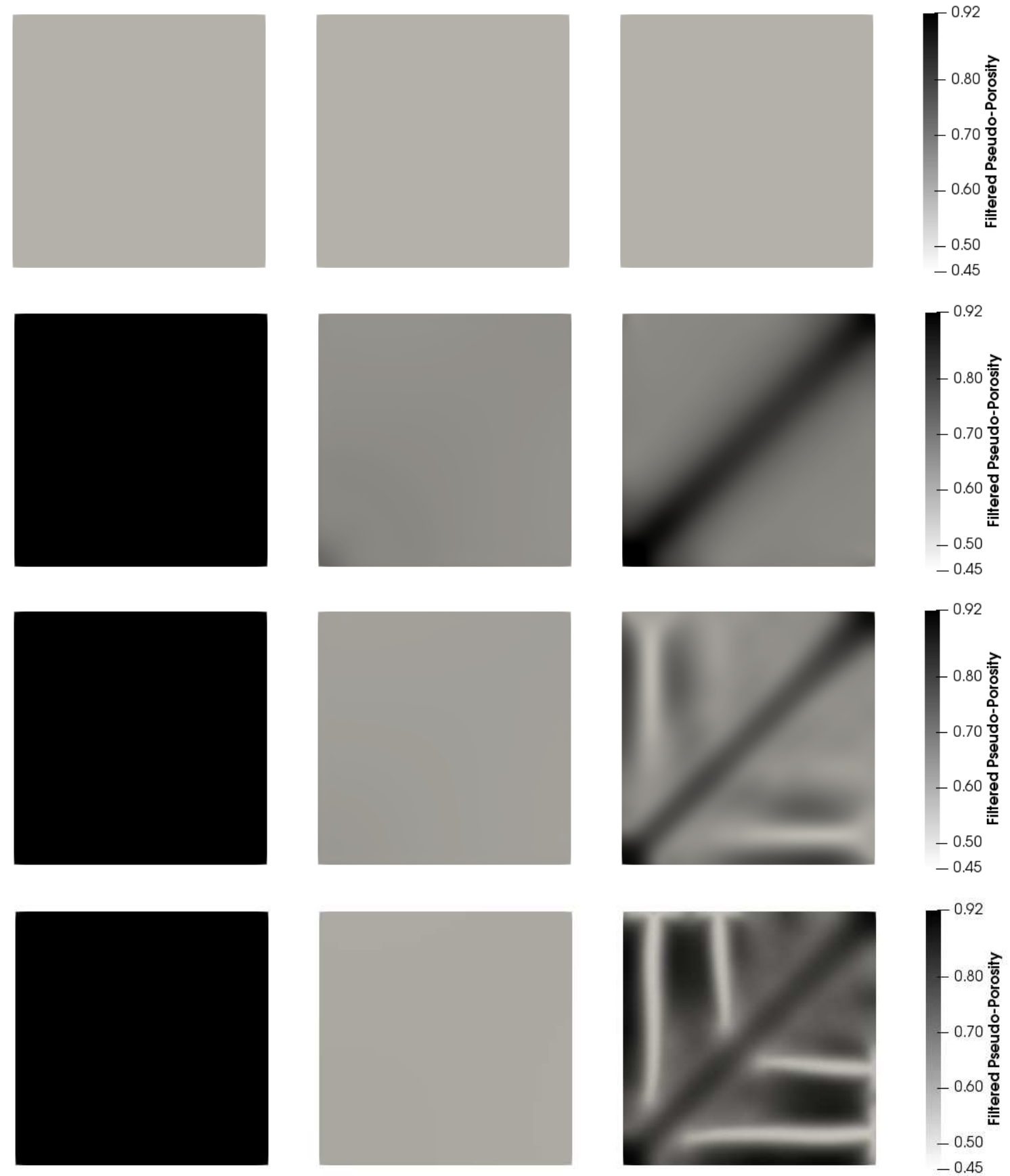

Figure 5.1: Pseudo-porosity fields evolution (top-down) for SIMP (left), non-offset hyperbolic tangents (centre) and offset hyperbolic tangents (right) material models for prismatic tank optimisation. 


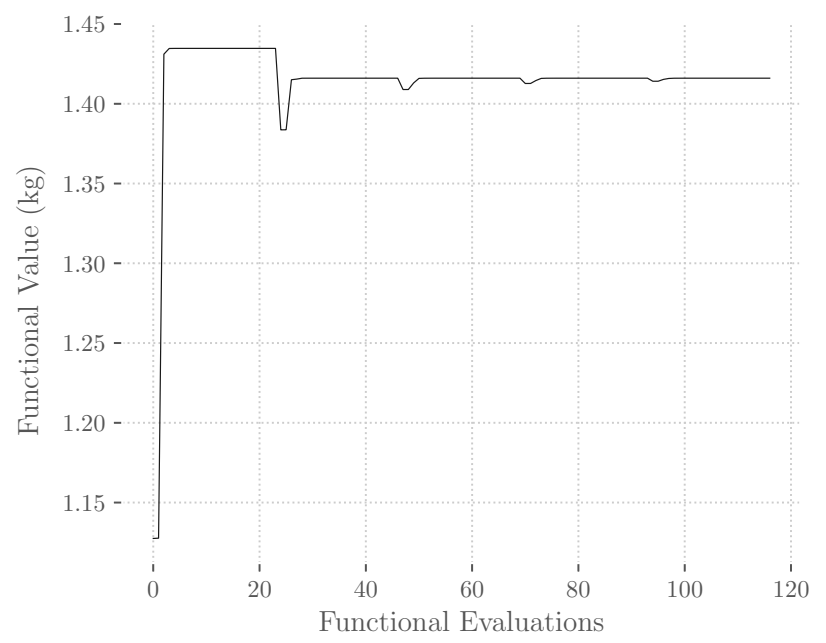

(a) SIMP

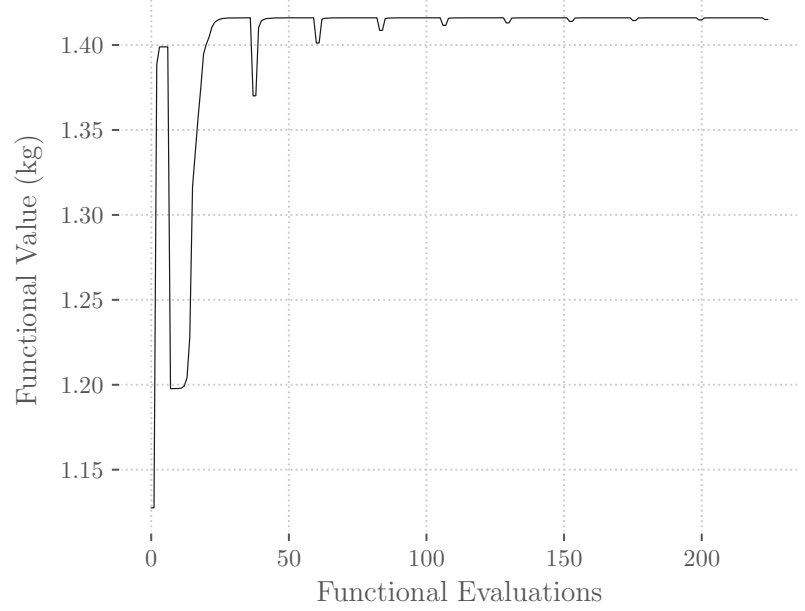

(b) Non-offset hyperbolic tangents

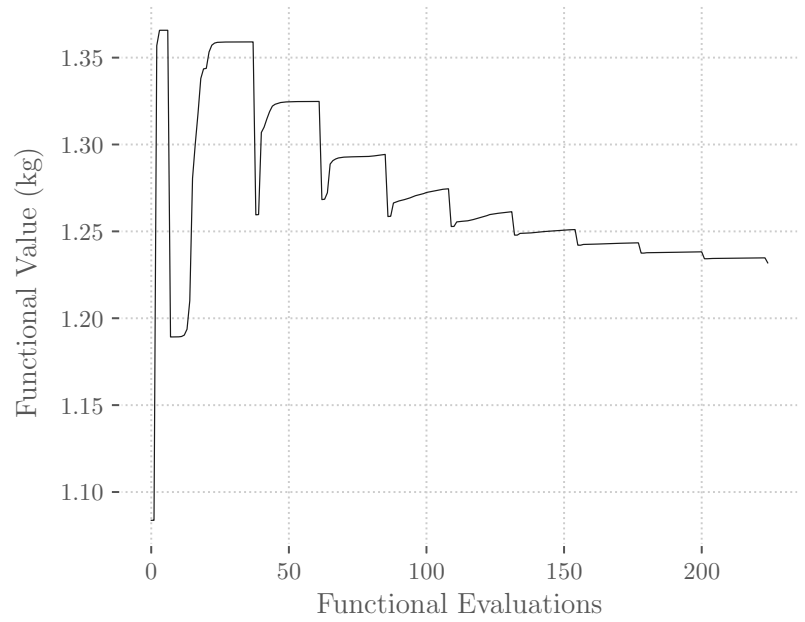

(c) Offset hyperbolic tangents

Figure 5.2: Functional convergence for different material models. 


\subsubsection{Dependency on the Initial Guess and Penalisation}

Topology optimisation problems are non-convex and often lead to different solutions depending on the initial guess and on the strategy adopted for the penalisation continuation on the OHT material model. This Section is limited to the numerical study of such dependency, while the subsequent Sections are focused on the actual design of ANG tanks.

Let consider the optimisation of the prismatic tank defined in Table 5.5. Figure 5.3 demonstrates the independency of the final topology with respect to the initial guess. The evolution of the optimisation starting from an homogeneous pseudo-porosity distribution $\mathbf{e}=\mathbf{0 . 5}$ is shown on the left column while one starting from an arbitrary initial guess is shown on the right. In both cases, the distribution of pseudo-porosities goes towards a fullcarbon domain during the first optimisation iterations, following an identical evolution after that toward the same final layout. The optimisation of this tank is resumed in Section 5.4.1, where a thorough analysis is presented.

$$
\begin{aligned}
\begin{array}{r}
\text { Domain } \\
\text { Heat Convection: }
\end{array} & h_{\Gamma_{W}}=100 \mathrm{Wm}^{-2} \mathrm{~K}^{-1} \\
\text { Cycle } & \\
\text { Time Length: } & t_{\text {fin,a }}=10800 \mathrm{~s} \text {, Fig. }(3.3) \\
\text { Optimisation } & \\
\text { Material Model: } & \epsilon=0.02, \mathrm{Eq} .(3.13) \\
& p=[1,51], \Delta p=5 \\
\text { Iterations per Step: } & i t_{\max }=20 \\
\text { Tolerance: } & \omega_{\min }=1.0 \times 10-6 \\
\text { Filter Radius: } & z=5 \times 10^{-3} \mathrm{~m}, \text { Eq. }(4.44)
\end{aligned}
$$

Prismatic Tank

(Table 5.1)

Table 5.5: Optimisation definitions for a prismatic tank subjected to forced convection.

The cylindrical tank optimisation presented in Table 5.6 is ran from a material model which is already significantly penalised $\left(p_{i n i}=20\right)$. In this case, different initial guesses result in different final layouts as seen in Figure 5.4, where the distributions on the top of each block show the evolution of the homogeneous initial guess, while the bottom ones refers to an arbitrary initial distribution of pseudo-porosity. Despite the different layouts obtained for each case, the final objective function values are very similar: $2.31 \mathrm{~kg}$ of total 

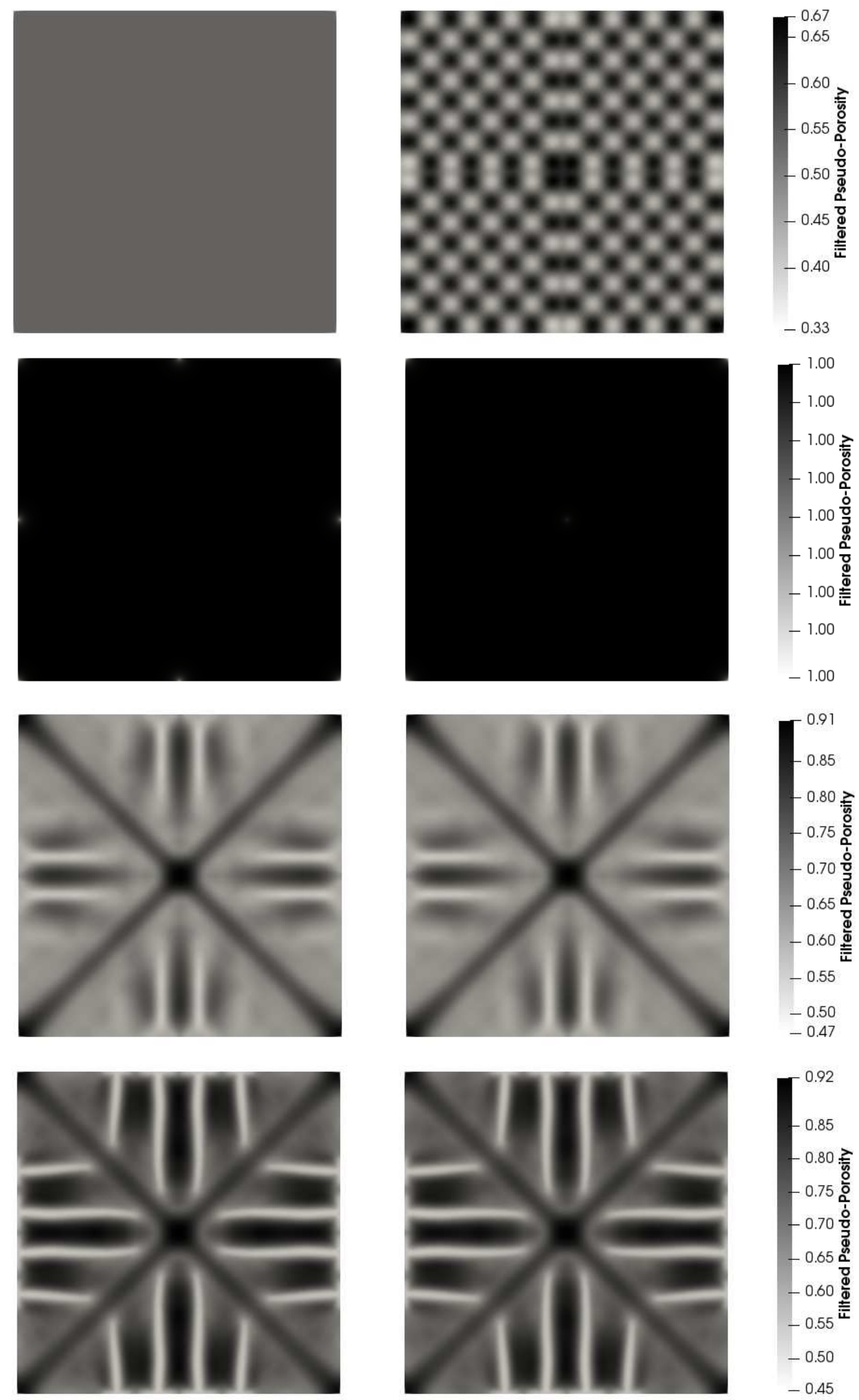

Figure 5.3: Pseudo-porosity fields evolution for homogeneous (left) and arbitrary (right) initial guesses for prismatic tank optimisation starting with $p_{i n i}=1$. 
gas intake for the homogeneous initial guess and $2.28 \mathrm{~kg}$ for the arbitrary.

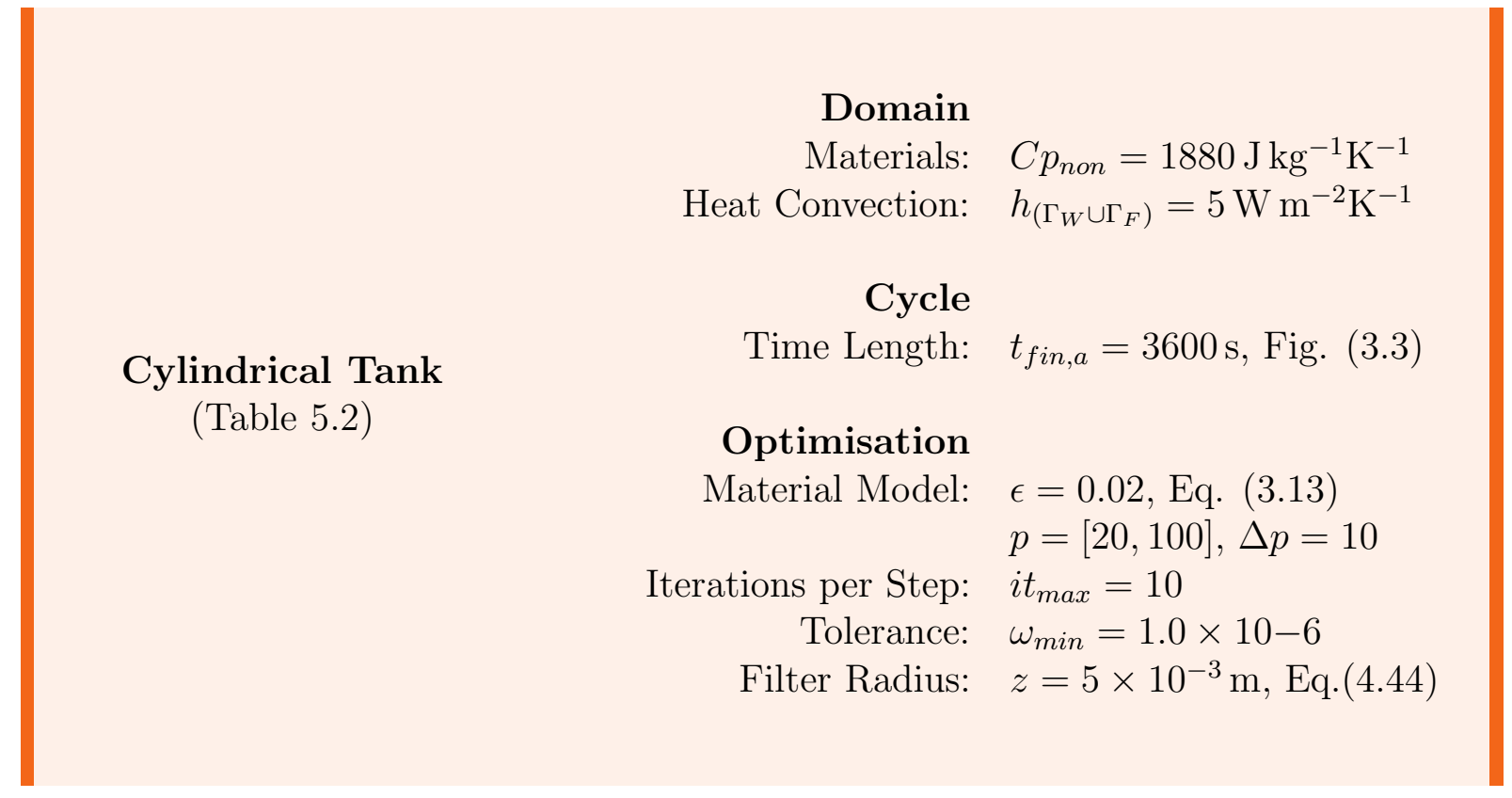

Table 5.6: Optimisation definitions for a cylindrical domain with non-adsorbent material with high heat capacity.

The dependence of the final layouts on the initial guesses can be mitigated by following a continuation approach starting from a material model presenting low penalisation. Figure 5.5 shows the evolution of the pseudo-porosity distribution for an optimisation starting with penalisation $p_{i n i}=1$. Similar to the prismatic case, shown in Figure 5.3, the initial optimisation steps take the pseudo-porosities to a full-carbon distribution, following the same evolution from then on. The final layout for this case results in a total gas intake of $2.30 \mathrm{~kg}$, which is very similar to the $2.31 \mathrm{~kg}$ observed for the case starting the penalisation from $p_{i n i}=20$. This particular case also highlights the non-convexity of such optimisation problem, where a particular distribution of pseudo-porosities in a moderately penalised material model complies the objective function better than a less penalised one following a smooth continuation. The optimisation of this tank is approached in detail in Section 5.3.3.

\subsection{Design of ANG Tanks with Passive Thermal Man- agement}

In this Section, tanks filled without the aid of any external work for managing the temperature of the adsorbent bed are studied. 

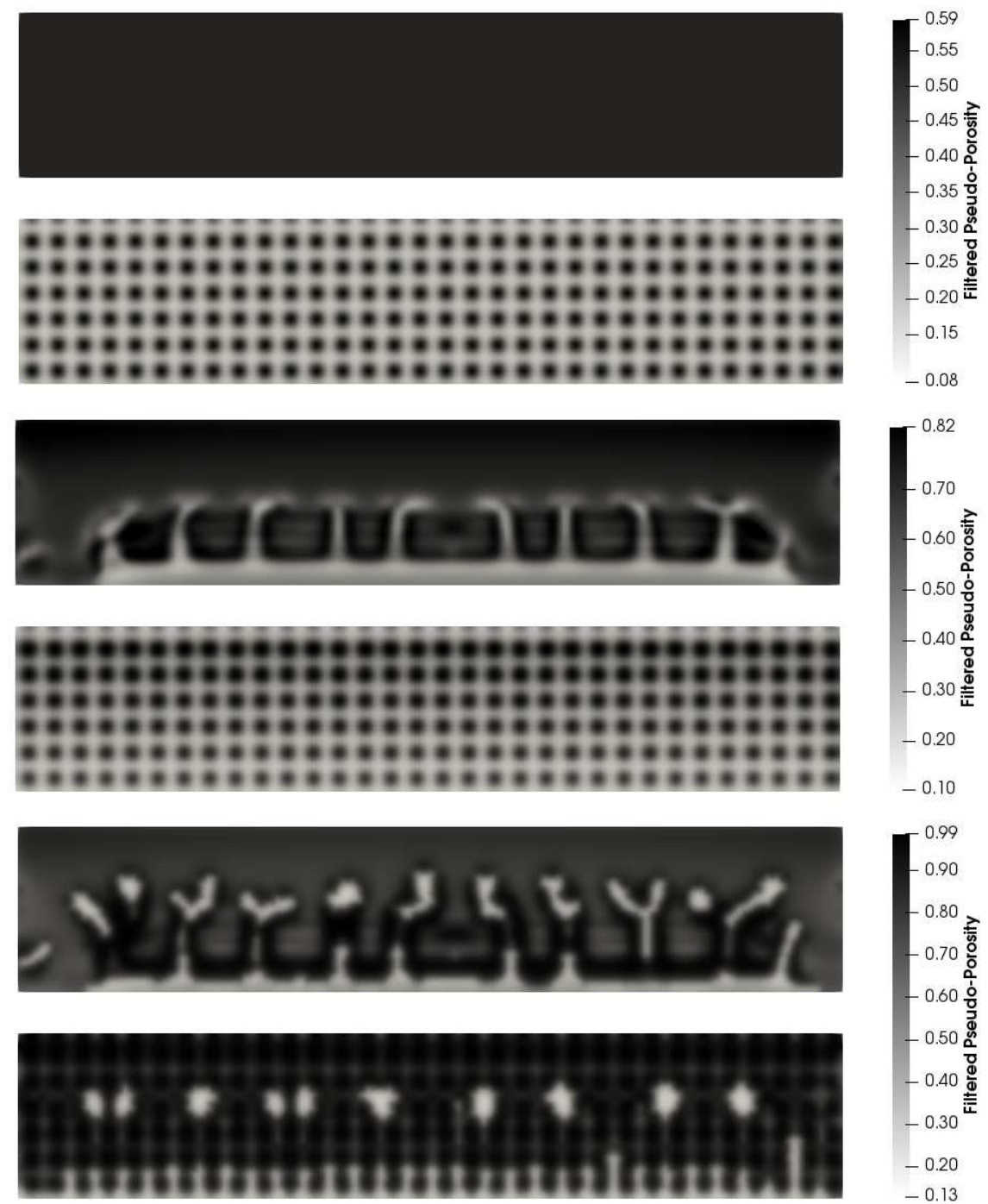

Figure 5.4: Pseudo-porosity fields evolution for homogeneous (top image on each block) and arbitrary (bottom) initial guesses for cylindrical tank optimisation starting with $p_{i n i}=$ 20 . 

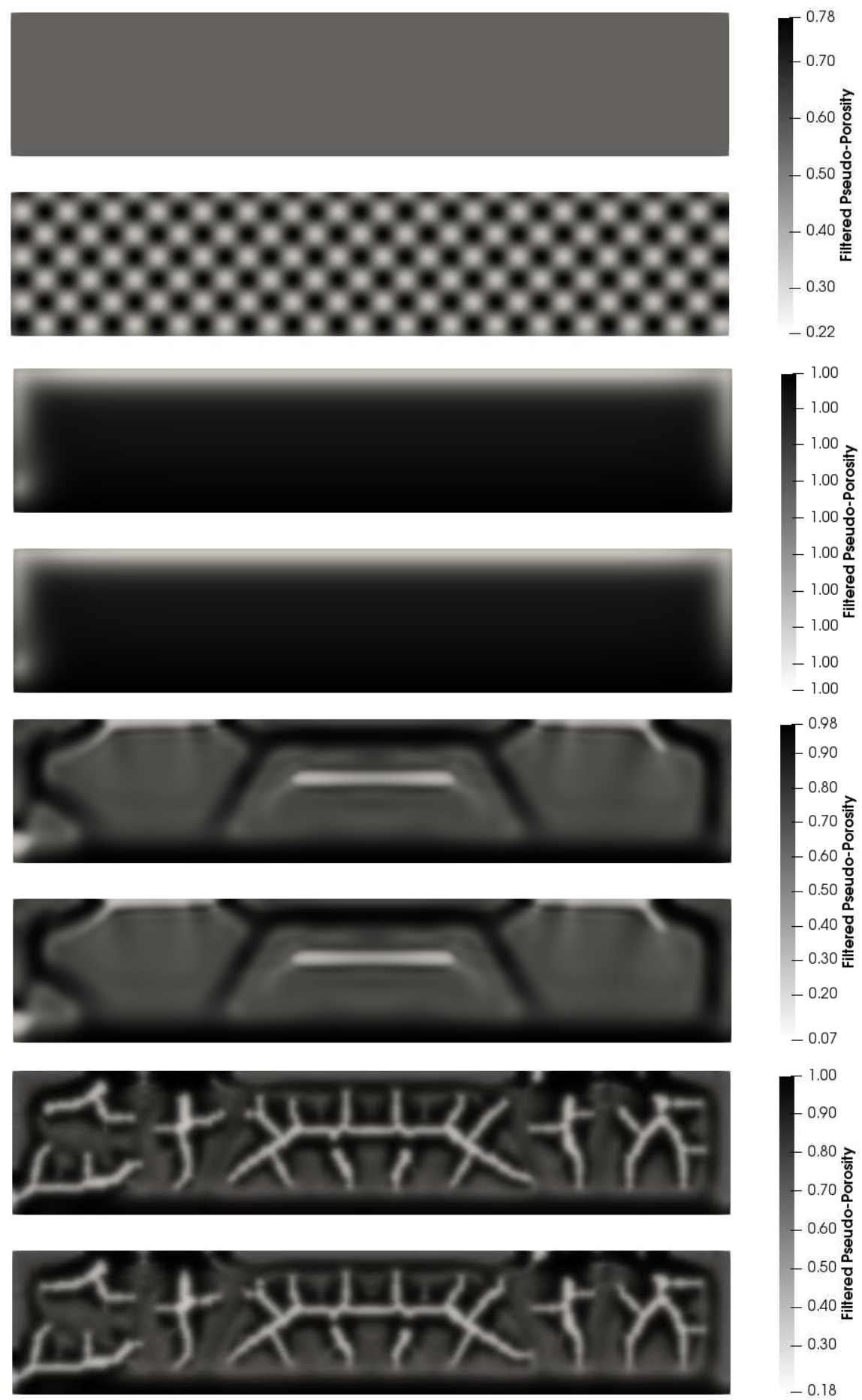

Figure 5.5: Pseudo-porosity fields evolution for homogeneous (top image on each block) and arbitrary (bottom) initial guesses for cylindrical tank optimisation starting with $p_{i n i}=$ 1. 


\subsubsection{Tanks Subjected to Natural Convection}

The optimisation of a cylindrical tank subjected to natural convectionis is defined in 5.7. It starts from an homogeneous distribution of the design variables at $\mathbf{e}=\mathbf{0 . 5}$ and the penalisation is smoothly increased from $p_{\text {ini }}=20$ to $p_{\max }=100$, following the flowchart shown in Figure 4.15 with $i t_{\max }=10$ and $\omega_{\min }=1.0 \times 10-6$. The pseudo-porosity distribution is subjected to Helmholtz filtering with radius $z=5 \times 10^{-3}$, which is equal to the double of the characteristic mesh cell diameter.

In Figure 5.6, the resulting topology is shown along with its three-dimensional revolution. The distribution of the pseudo-porosities obtained from the optimiser is shown in Figure 5.6a, where the colour scale ranges from grey, representing Steel $(\mathrm{e}=0.0)$, to black, representing Activated Carbon $(\mathrm{e}=1.0)$. Given the properties of the material model adopted, the interpretation of binary distribution of materials inside the domain is made on the 0.5 threshold. Figure $5.6 \mathrm{~b}$ shows the convergence of the objective function, which presents a very smooth behaviour. The total density of gas, that is, the sum of gaseous and adsorbed phase density, is shown in Figure 5.6c for a domain fully occupied by Carbon $(\mathbf{e}=\mathbf{1 . 0})$ and for the optimised distribution of Steel. It can be seen that the gas inlet was reduced approximately in $3 / 4$ in order to allow the growth of a metallic region along the center of tank extending the zone of high density of gas adsorbed, which was before limited to the inlet neighbourhood. It is due to the fact that the gas entering the domain is at room temperature $(293 \mathrm{~K})$ and creates a region of low temperature, which was extended because of the higher thermal conductivity of the Steel in relation to Activated Carbon, as shown in the temperature distribution plotted in Figure 5.6d. However, the increase of the gas density in this zone comes with the loss of adsorbent volume, as detailed in Section 3.1, making the net gain in the total mass of gas admitted being only $1.06 \%$. 
Domain

Heat Convection: $\quad h_{\left(\Gamma_{W} \cup \Gamma_{F}\right)}=5 \mathrm{~W} \mathrm{~m}^{-2} \mathrm{~K}^{-1}$

Cycle

Time Length: $\quad t_{\text {fin }, a}=10800 \mathrm{~s}$, Fig. (3.3)

Cylindrical Tank

(Table 5.2)

Optimisation

Material Model: $\epsilon=0.02$, Eq. (3.13)

$p=[20,100], \Delta p=10$

Iterations per Step: $i t_{\max }=10$

Tolerance: $\quad \omega_{\min }=1.0 \times 10-6$

Filter Radius: $z=5 \times 10^{-3} \mathrm{~m}$, Eq.(4.44)

Table 5.7: Optimisation definitions for a cylindrical domain subjected to natural convection. 


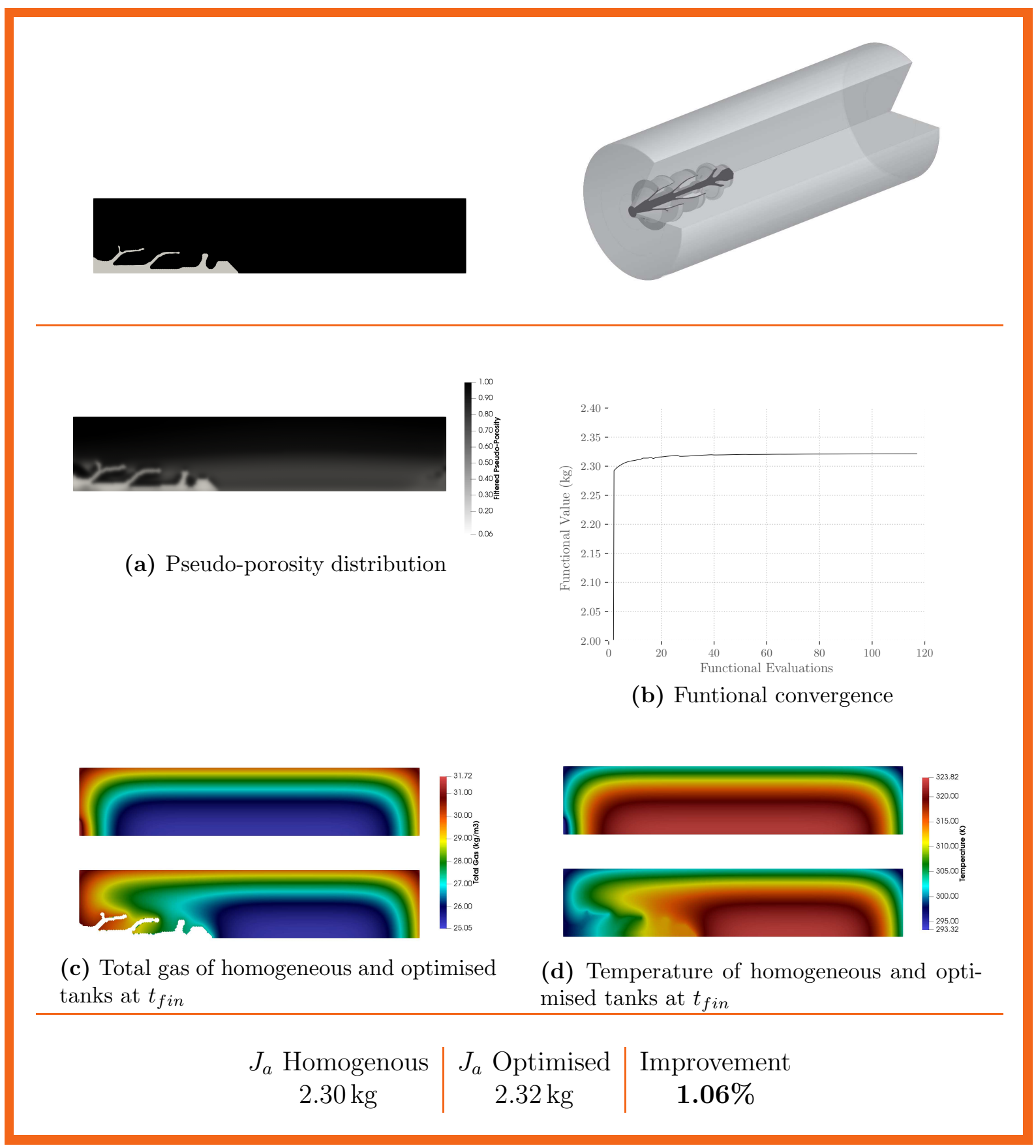

Figure 5.6: Optimised design for a cylindrical domain subjected to natural convection. 


\subsubsection{Sensitivity to Thermal Conductivity}

The non-adsorbent material considered when designing ANG tanks is not restricted to Steel only. Other metals with higher thermal conductivity, such as Aluminium, may be suitable in some cases. Aiming to verify that, a hypothetical material presenting conductivity four times higher than Steel is considered in the cycle defined in Table 5.8. Figure 5.7 shows a clear increase in the complexity of the metallic region connected to the inlet. Besides advancing further along the domain, this region presents more and thinner legs when compared to the optimisation performed with standard Steel properties - Figure 5.6. A new non-adsorbent region is also created from the wall opposite to the gas inlet, which would not compensate the loss in volume in the previous case. Figure 5.7d shows a clear reduction of the overall temperature within the domain, resulting in a net gain of $2.13 \%$ in the total mass of gas admitted. Therefore, the increase of the thermal conductivity of the non-adsorbent material has a positive impact on the optimisation of the ANG tanks considered in this work.

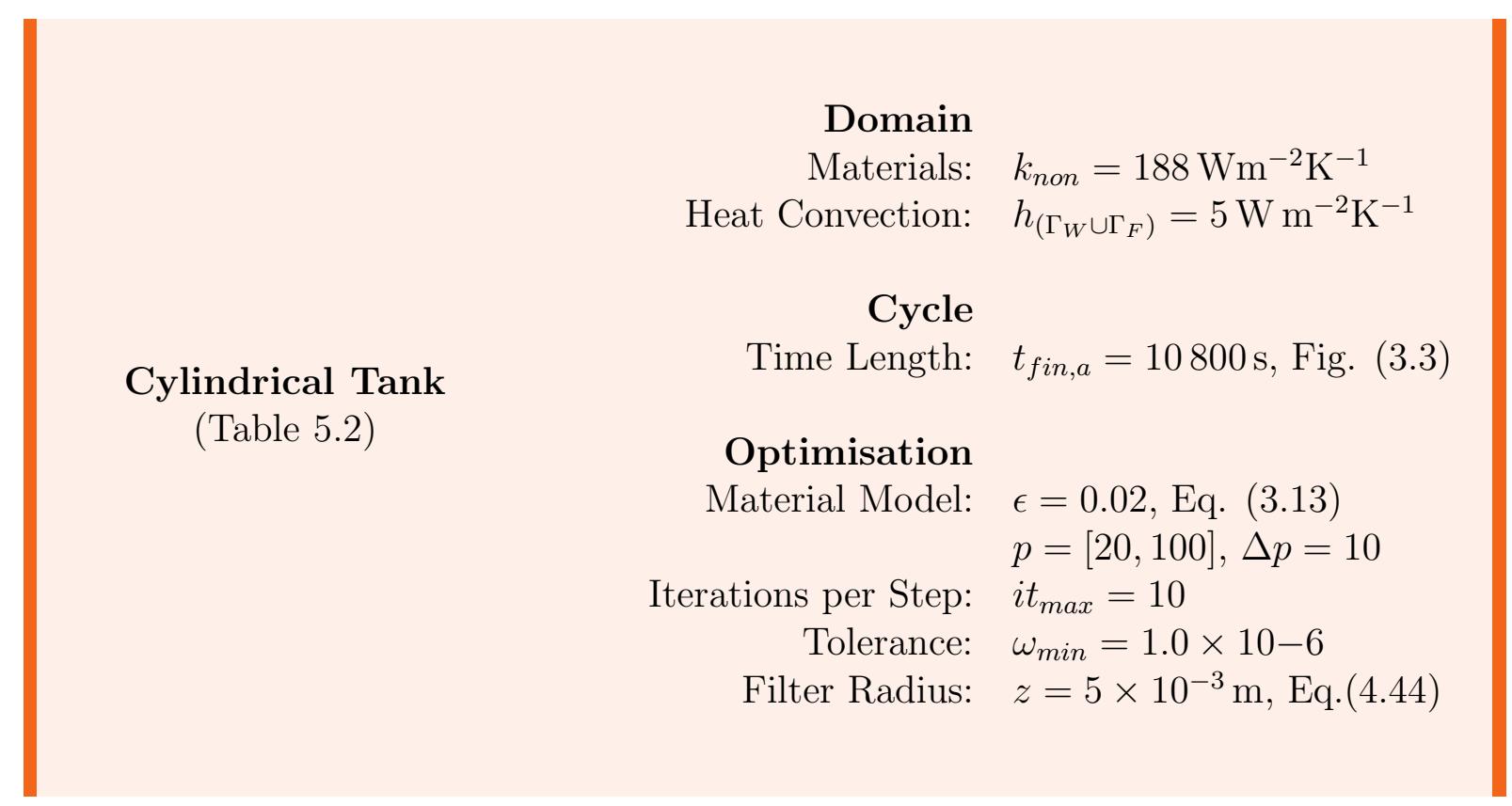

Table 5.8: Optimisation definitions for a cylindrical domain with non-adsorbent material with high thermal conductivity. 


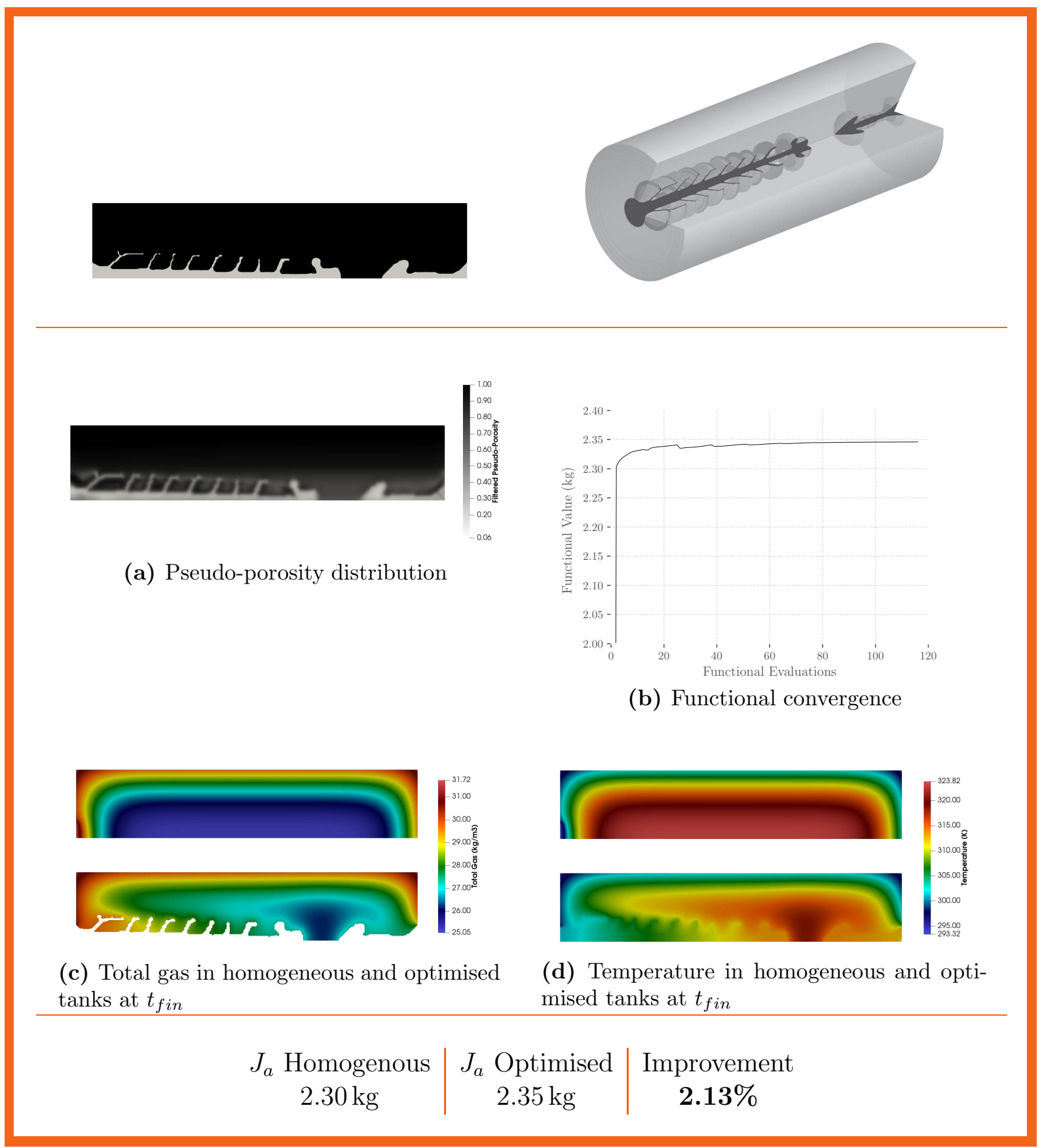

Figure 5.7: Optimised design for a cylindrical domain with non-adsorbent material with high thermal conductivity. 


\subsubsection{Sensitivity to Heat Capacity}

Extending the previous Section, the impact of the increase of the heat capacity of the non-adsorbent material is studied here. Materials presenting enough high heat capacity can be used to roughly approximate the impact of the insertion of Phase Change Materials (PCM) regions inside the domain - see Toledo et al. (2013) for more details about PCM operation. The specific heat capacity of Steel is multiplied by four, resulting in the problem defined in Table 5.9. Figure 5.8 shows the resulting topology for this case, where the non-adsorbent regions are not necessarily connected to the gas inlet or to the walls. The occurrence of this layout is due to the fact that materials with high heat capacity act as heat sinks. This approach seems more promising than using high conductive materials as it is reflected in the high gas density through the whole domain observed in Figure 5.8c. In the comparison with the homogeneous case, the the amount of gas admitted during the filling cycle is $6.30 \%$ higher when compared with the domain filled with Carbon only. The relative gain is even higher for shorter cycles. Table 5.10 defines a cycle lasting 3600 s, keeping all the other parameters the same. It can be seen in Figure 5.9 that more non-adsorbent islands were formed in order to take advantage of its high heat capacity. Such islands represent hoops as depicted in the revolved three-dimensional topology. It is important to highlight that this optimised domain admits more gas in $3600 \mathrm{~s}(2.31 \mathrm{~kg})$ than the homogeneous does in $10800 \mathrm{~s}(2.30 \mathrm{~kg})$. This fact makes the use materials with high heat capacity very promising in fast-filling ANG tanks. Table 5.11 defines an even shorter cycle of $1200 \mathrm{~s}$. In Figure 5.10, it can be seen that the non-adsorbent islands becomes smaller and are located nearer the axis of symmetry, in order to reduce the loss in adsorbent volume. However, the improvement is much smaller than on the previous ones: $2.89 \%$. In this case, the cooling effect is constrained by the poor thermal conductivity of the Activated Carbon, given that the cycle is very short. That is, the non-adsorbent islands posses a very small radius of influence around them, as can be clearly seen in Figure 5.10d. 


$$
\begin{aligned}
\begin{array}{r}
\text { Domain } \\
\text { Materials: }
\end{array} & C p_{\text {non }}=1880 \mathrm{Jkg}^{-1} \mathrm{~K}^{-1} \\
\text { Heat Convection: } & h_{\left(\Gamma_{W} \cup \Gamma_{F}\right)}=5 \mathrm{~W} \mathrm{~m}^{-2} \mathrm{~K}^{-1} \\
\text { Cycle } & \\
\text { Time Length: } & t_{\text {fin, },}=10800 \mathrm{~s}, \text { Fig. }(3.3) \\
\text { Optimisation } & \\
\text { Material Model: } & \epsilon=0.02, \mathrm{Eq} .(3.13) \\
& p=[20,100], \Delta p=10 \\
\text { Iterations per Step: } & i t_{\max }=10 \\
\text { Tolerance: } & \omega_{\min }=1.0 \times 10-6 \\
\text { Filter Radius: } & z=5 \times 10^{-3} \mathrm{~m}, \mathrm{Eq} \cdot(4.44)
\end{aligned}
$$

Cylindrical Tank

(Table 5.2)

Table 5.9: Optimisation definitions for a cylindrical domain with non-adsorbent material with high heat capacity.

\section{Domain}

Materials: $\quad C p_{\text {non }}=1880 \mathrm{Jkg}^{-1} \mathrm{~K}^{-1}$

Heat Convection: $\quad h_{\left(\Gamma_{W} \cup \Gamma_{F}\right)}=5 \mathrm{~W} \mathrm{~m}^{-2} \mathrm{~K}^{-1}$

\section{Cycle}

Cylindrical Tank

(Table 5.2)

Time Length: $\quad t_{f i n, a}=3600 \mathrm{~s}$, Fig. $(3.3)$

$$
\begin{aligned}
\text { Optimisation } & \\
\text { Material Model: } & \epsilon=0.02, \mathrm{Eq} .(3.13) \\
& p=[20,100], \Delta p=10 \\
\text { Iterations per Step: } & i t_{\max }=10 \\
\text { Tolerance: } & \omega_{\min }=1.0 \times 10-6 \\
\text { Filter Radius: } & z=5 \times 10^{-3} \mathrm{~m}, \text { Eq.(4.44) }
\end{aligned}
$$

Table 5.10: Optimisation definitions for a cylindrical domain with non-adsorbent material with high heat capacity in 3600 s filling cycle. 


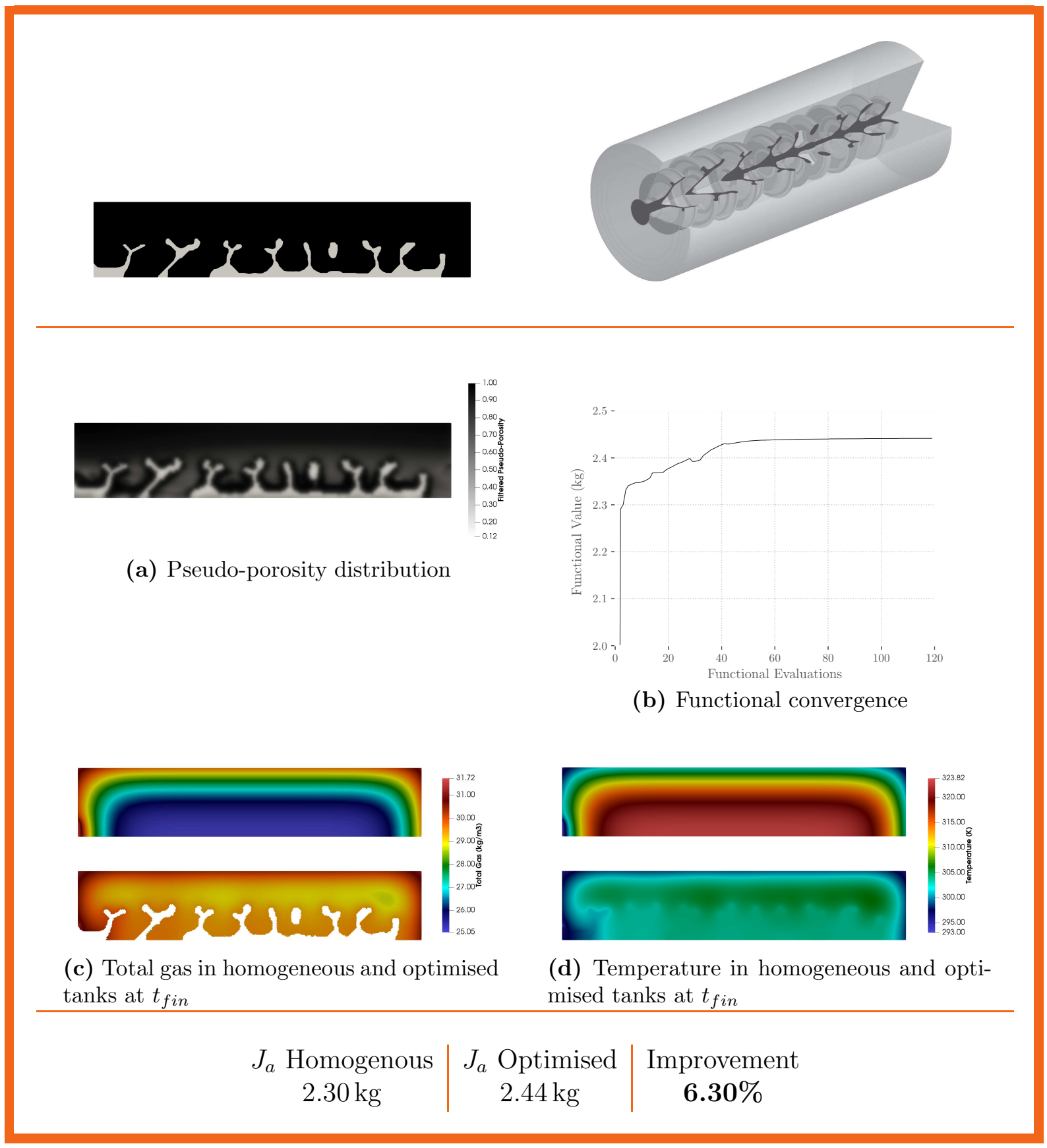

Figure 5.8: Optimised design for a cylindrical domain with non-adsorbent material with high heat capacity. 


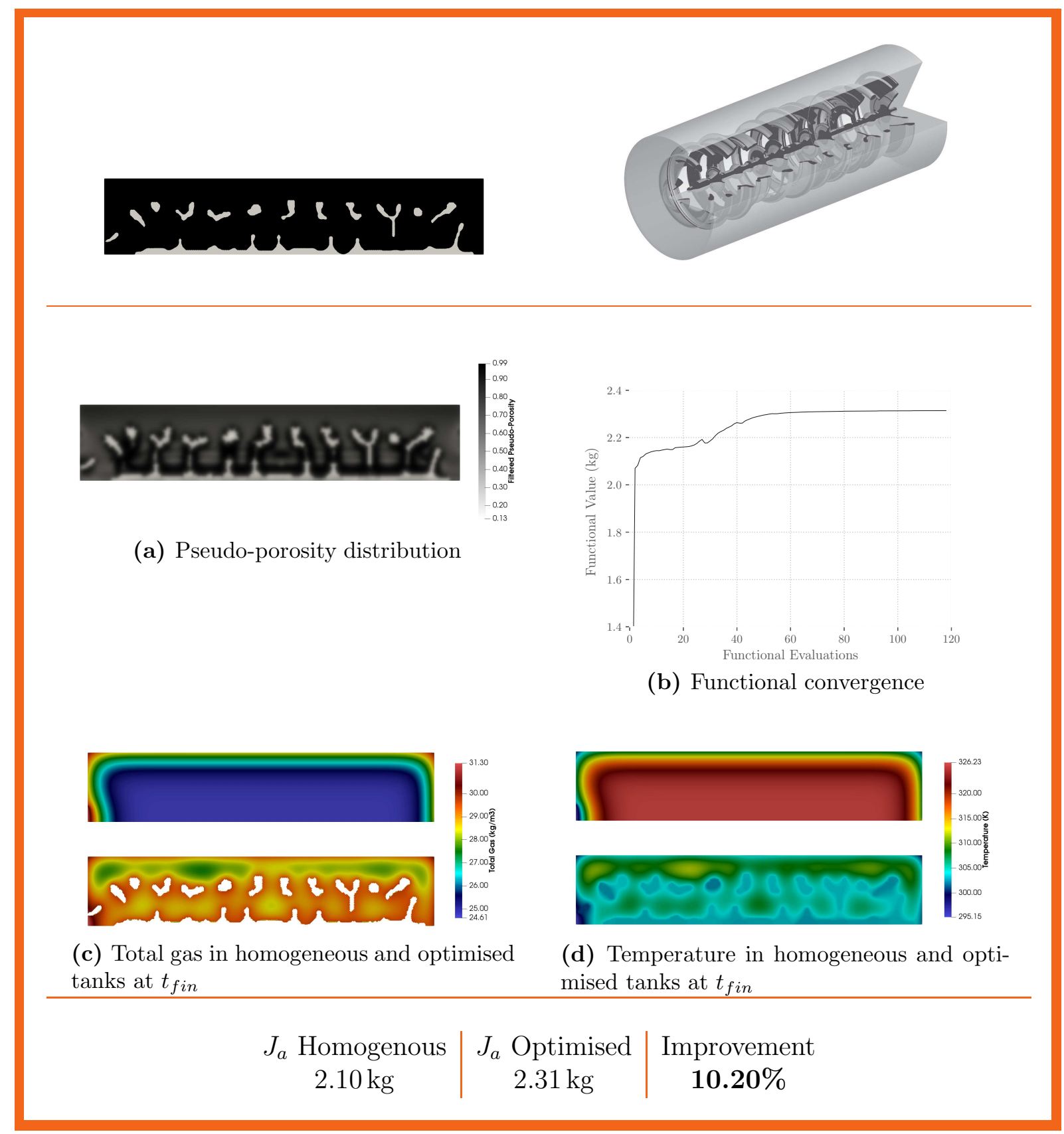

Figure 5.9: Optimised design for a cylindrical domain with non-adsorbent material with high heat capacity in 3600 s filling cycle. 


\section{Domain}

Materials: $\quad C p_{\text {non }}=1880 \mathrm{Jkg}^{-1} \mathrm{~K}^{-1}$

Heat Convection: $\quad h_{\left(\Gamma_{W} \cup \Gamma_{F}\right)}=5 \mathrm{~W} \mathrm{~m}^{-2} \mathrm{~K}^{-1}$

\section{Cycle}

Cylindrical Tank

(Table 5.2)

Time Length: $\quad t_{f i n, a}=1200$ s, Fig. (3.3)

$$
\begin{aligned}
\text { Optimisation } & \\
\text { Material Model: } & \epsilon=0.02, \mathrm{Eq} .(3.13) \\
& p=[20,100], \Delta p=10 \\
\text { Iterations per Step: } & i t_{\max }=10 \\
\text { Tolerance: } & \omega_{\min }=1.0 \times 10-6 \\
\text { Filter Radius: } & z=5 \times 10^{-3} \mathrm{~m}, \text { Eq.(4.44) }
\end{aligned}
$$

Table 5.11: Optimisation definitions for a cylindrical domain with non-adsorbent material with high heat capacity in $1200 \mathrm{~s}$ filling cycle. 


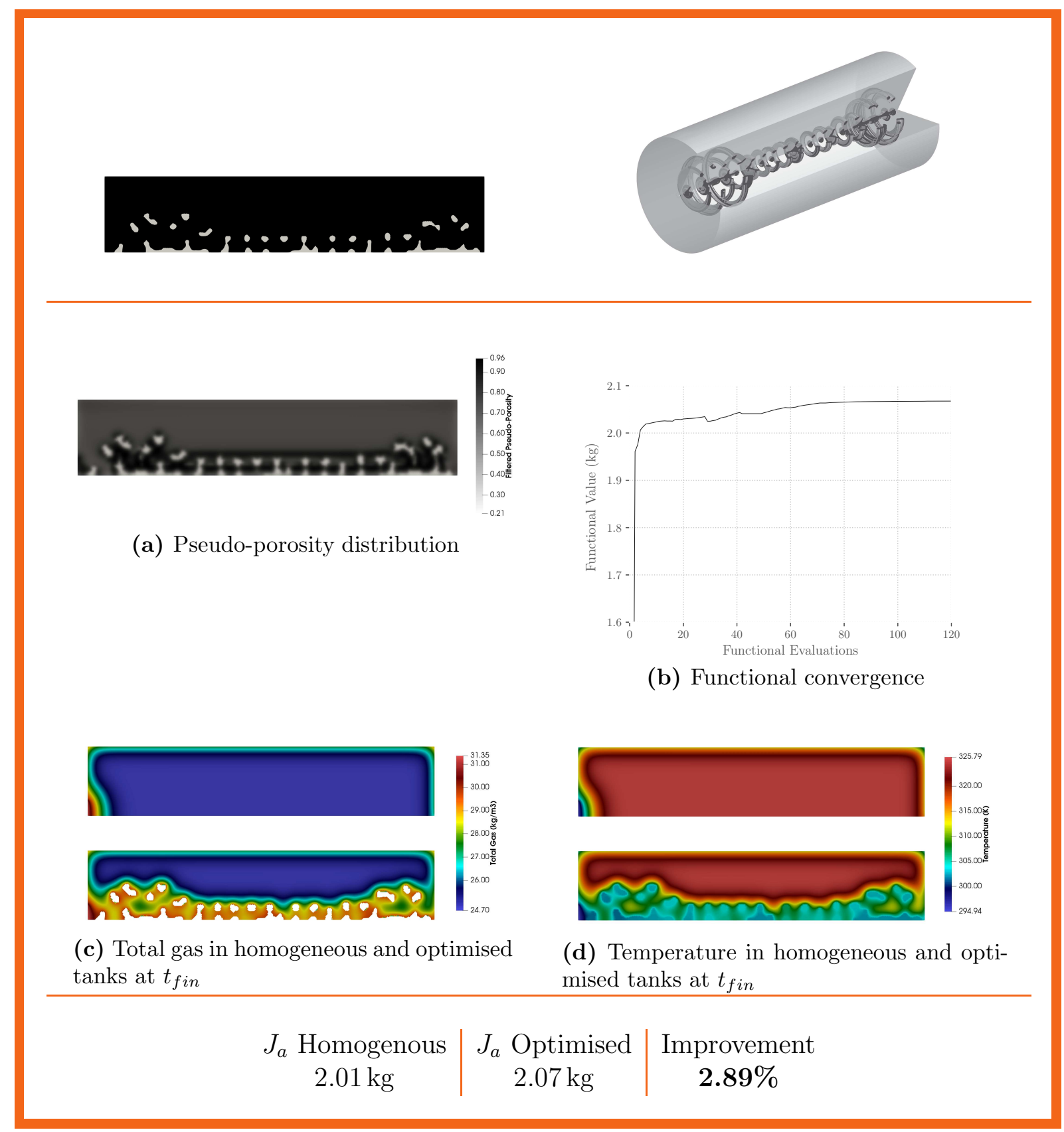

Figure 5.10: Optimised design for a cylindrical domain with non-adsorbent material with high heat capacity in 1200 s filling cycle. 


\subsection{Design of ANG Tanks with Active Thermal Man- agement}

This Section considers tanks filled with the aid of some external work for the thermal management, such as the imposition of forced convection or low temperature at some point of the domain. The latter approach is adopted in Vasiliev et al. (2014), where fins linked to a very cold wall, at $233 \mathrm{~K}$, are distributed radially along an adsorbent cylinder.

\subsubsection{Tanks Subjected to Forced Convection}

The resulting topology depicted in Figure 5.11 shows perfectly symmetric fins connecting the central region of the section to the walls subjected to forced convection. Such symmetry is due to the use of a regular mesh constituted by equal elements. Figure 5.11d shows that the fins made the maximum temperature inside the domain drop from $325 \mathrm{~K}$ to $310 \mathrm{~K}$, allowing $5.46 \%$ more gas to be admitted during the filling cycle. An instability is seen on Figure 5.11b for the convergence of the objective function due to the increase of the penaliser from $p_{\text {ini }}=20$ to $p=30$.

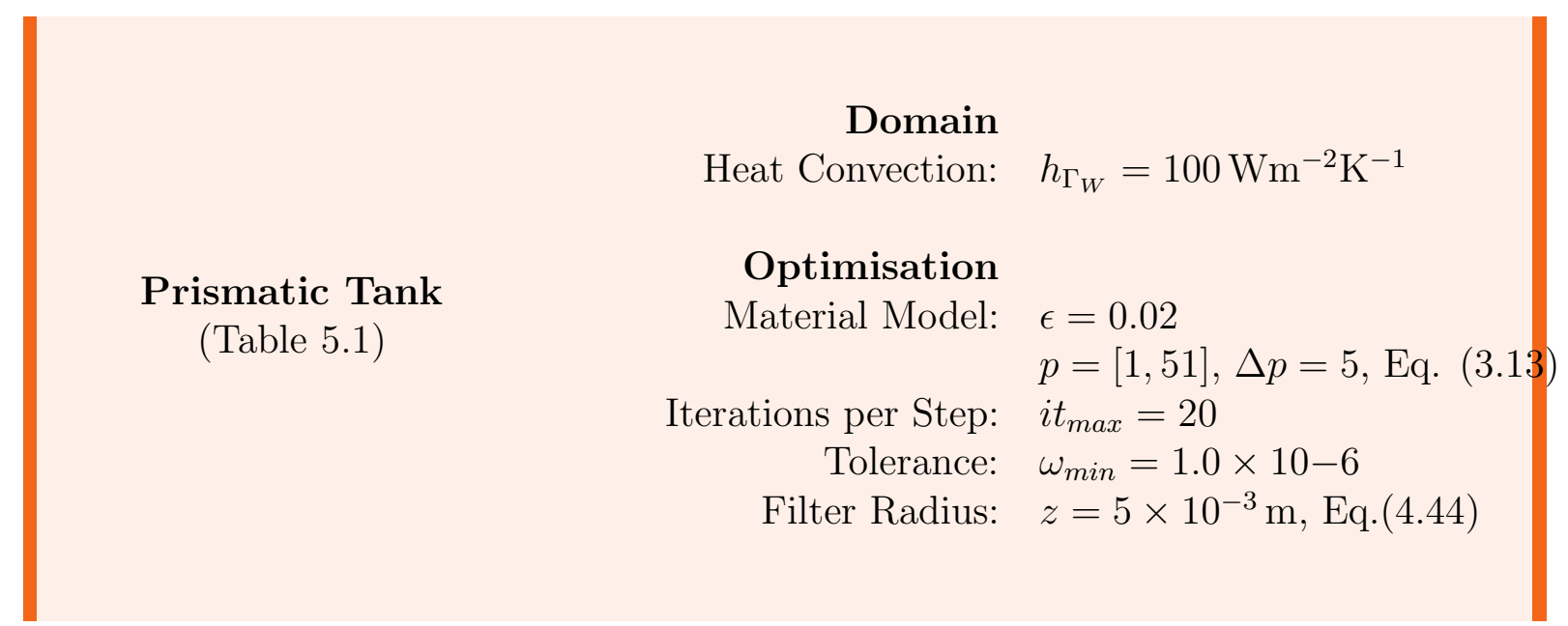

Table 5.12: Optimisation definitions for a prismatic domain subjected to forced convection. 

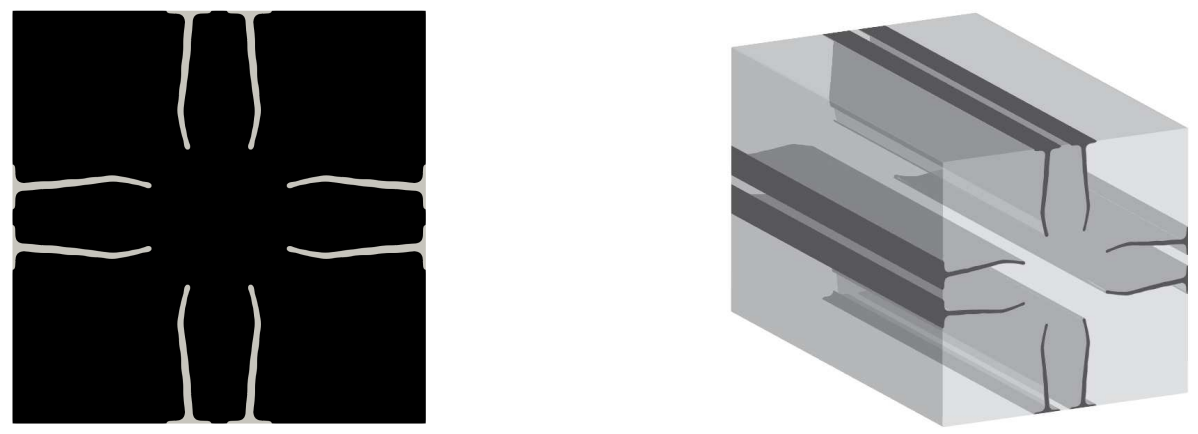

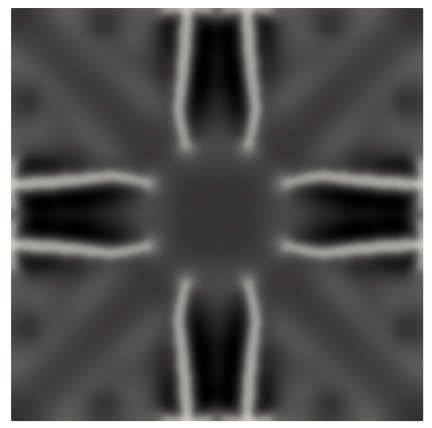

(a) Pseudo-porosity distribution
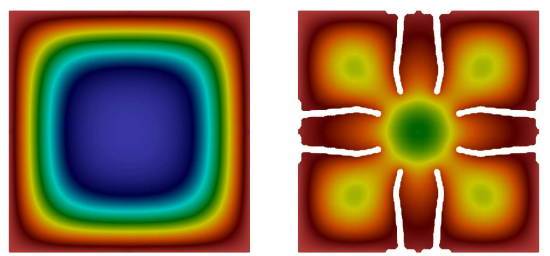

(c) Total gas in homogeneous and optimised tanks at $t_{f i n}$

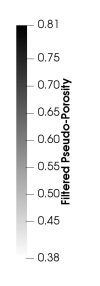

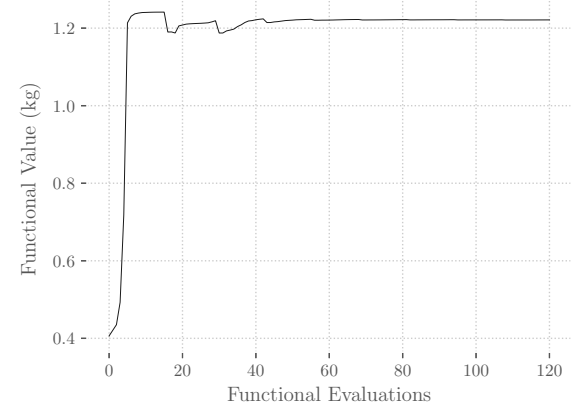

(b) Functional convergence
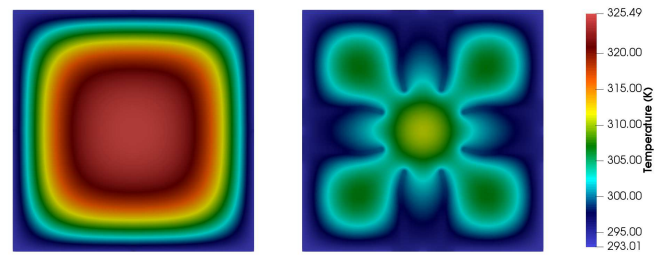

(d) Temperature in homogeneous and optimised tanks at $t_{\text {fin }}$

$$
\begin{array}{c|c|c}
J_{a} \text { Homogenous } & J_{a} \text { Optimised } & \text { Improvement } \\
1.16 \mathrm{~kg} & 1.22 \mathrm{~kg} & \mathbf{5 . 4 6 \%}
\end{array}
$$

Figure 5.11: Optimised design for a prismatic domain subjected to forced convection. 


\subsubsection{Sensitivity to Forced Convection and Cold Walls}

In order to study the impact of different thermal boundary conditions, the complexshaped axisymmetric domain defined in 5.3 is adopted. Initially, forced convection is considered by imposing $h=100 \mathrm{Wm}^{-1} \mathrm{~K}^{-1}$, as defined in Table 5.13. The resulting topology shown in Figure 5.12 presents the trend of growing Steel fins from concave corners. Such behaviour is justified by the temperature distribution in the homogeneous case observed in Figure 5.12d. These corners are the regions with steepest temperature gradient from points on the wall towards the inside of the domain. Accordingly, the curved shape acquired by the fins coincide almost perfectly with the path of maximum gradient. Based on this approach, the optimised domain is able to admit $1.23 \%$ more gas than the homogeneous one. By increasing the convective coefficient to $h=700 \mathrm{Wm}^{-1} \mathrm{~K}^{-1}$, small changes are observed. The most prominent change in the topology shown in Figure 5.12 is the fork of one of the fins. However, a very reticent relative gain is obtained in relation to the first case: $1.94 \%$ in total gas mass admitted. Therefore, the energy required to increase the convective heat transfer in the second case is reasonable. The case defined in Table 5.15 keeps the natural convection on the walls expect for the region $\Gamma_{F}$, which is kept at the fixed temperature of $273 \mathrm{~K}$. As seen in Figure 5.14, the low temperature makes the corners on $\Gamma_{F}$ preferred for the creation of fins. In Figure 5.14c, it can be clearly seen that the vicinity of the fins connected to the cold wall becomes the zone presenting the highest densities of gas, improving the total gas admission during the filling cycle in $7.51 \%$ when compared to the homogeneous case. 


\title{
Domain
}

Heat Convection: $\quad h_{\left(\Gamma_{W} \cup \Gamma_{F}\right)}=100 \mathrm{~W} \mathrm{~m}^{-2} \mathrm{~K}^{-1}$

\section{Cycle}

Custom Axisymmetric Tank

(Table 5.3)

Time Length: $\quad t_{f i n, a}=3600 \mathrm{~s}$, Fig. (3.3)

\author{
Optimisation \\ Material Model: $\epsilon=0.02$, Eq. (3.13) \\ $p=[20,100], \Delta p=10$ \\ Iterations per Step: $i t_{\max }=10$ \\ Tolerance: $\quad \omega_{\min }=1.0 \times 10-6$ \\ Filter Radius: $z=5 \times 10^{-3} \mathrm{~m}$, Eq.(4.44)
}

Table 5.13: Optimisation definitions for a custom axisymmetric domain subjected to forced convection with $h=100 \mathrm{Wm}^{-2} \mathrm{~K}^{-1}$.

\section{Domain}

Heat Convection: $\quad h_{\left(\Gamma_{W} \cup \Gamma_{F}\right)}=700 \mathrm{~W} \mathrm{~m}^{-2} \mathrm{~K}^{-1}$

Cycle

Time Length: $\quad t_{f i n, a}=3600 \mathrm{~s}$, Fig. (3.3)

Custom Axisymmetric Tank

(Table 5.3)

\section{Optimisation}

Material Model: $\epsilon=0.02$, Eq. (3.13)

Iterations per Step:

$p=[20,100], \Delta p=10$

Tolerance: $\quad \omega_{\min }=1.0 \times 10-6$

Filter Radius: $z=5 \times 10^{-3} \mathrm{~m}$, Eq.(4.44)

Table 5.14: Optimisation definitions for a custom axisymmetric domain subjected to forced convection with $h=700 \mathrm{Wm}^{-2} \mathrm{~K}^{-1}$. 

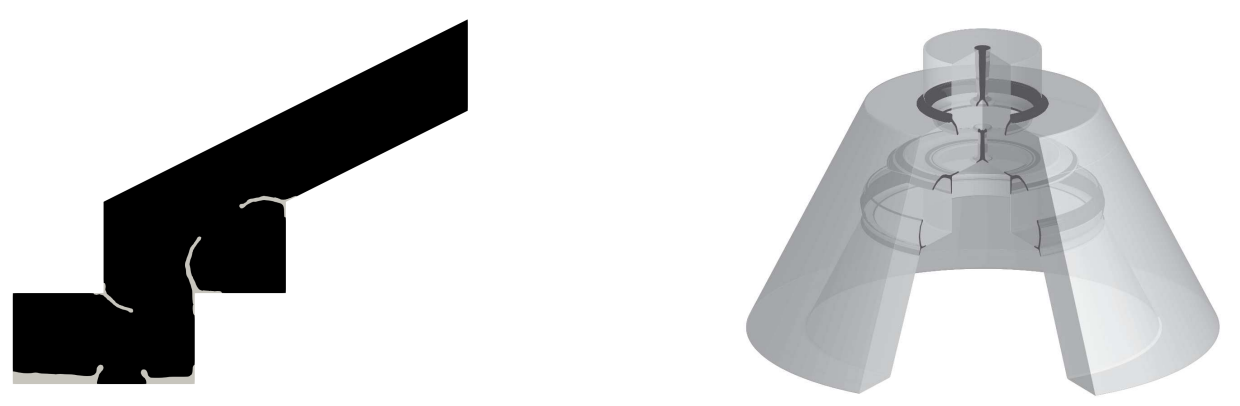

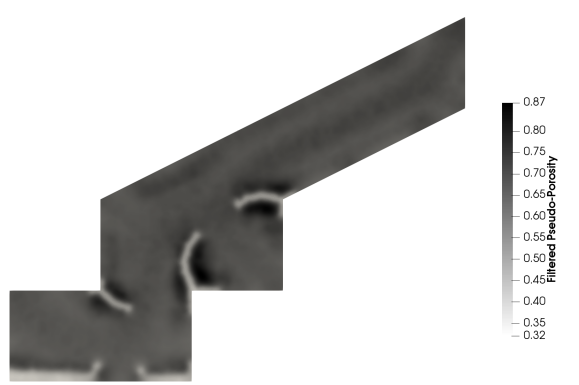

(a) Pseudo-porosity distribution

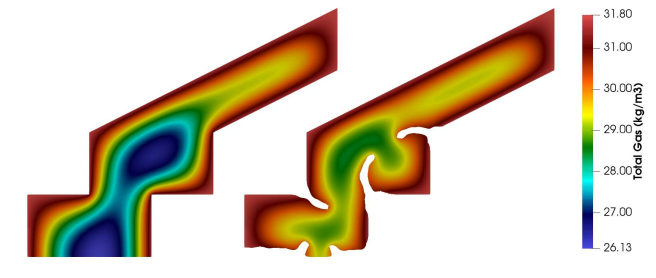

(c) Total gas in homogeneous and optimised tanks at $t_{\text {fin }}$

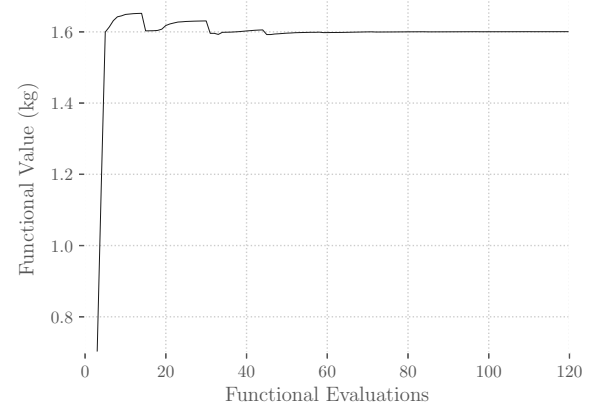

(b) Functional convergence

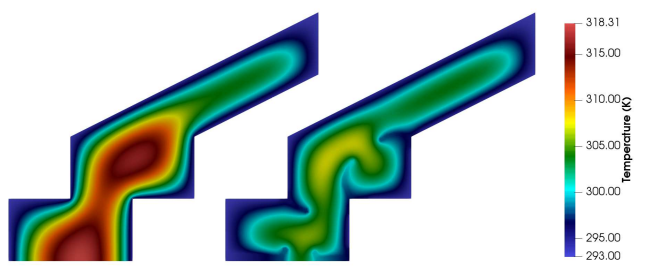

(d) Temperature in homogeneous and optimised tanks at $t_{\text {fin }}$

\section{\begin{tabular}{l|l|l}
$J_{a}$ Homogenous & $J_{a}$ Optimised & Improvement
\end{tabular} \begin{tabular}{l|l|l}
$1.58 \mathrm{~kg}$ & $1.60 \mathrm{~kg}$ & $\mathbf{1 . 2 3 \%}$
\end{tabular}}

Figure 5.12: Optimised design for a custom axisymmetric domain subjected to forced convection with $h=100 \mathrm{Wm}^{-2} \mathrm{~K}^{-1}$. 

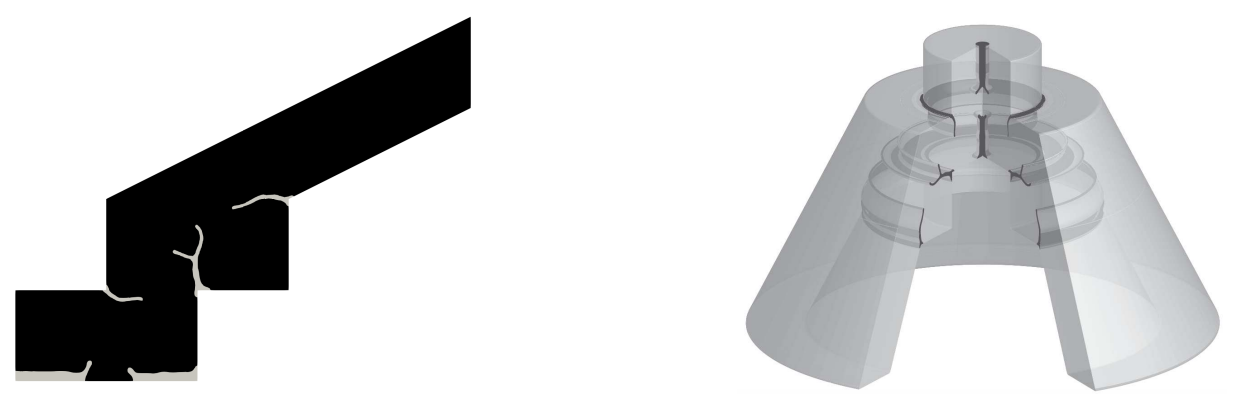

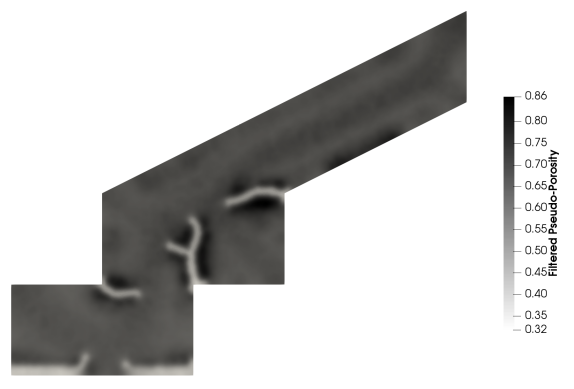

(a) Pseudo-porosity distribution

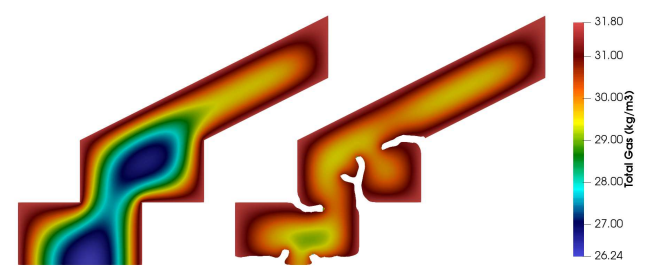

(c) Total gas in homogeneous and optimised tanks at $t_{\text {fin }}$

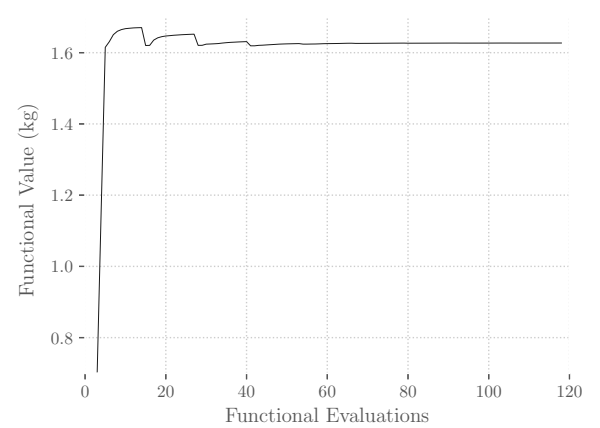

(b) Functional convergence

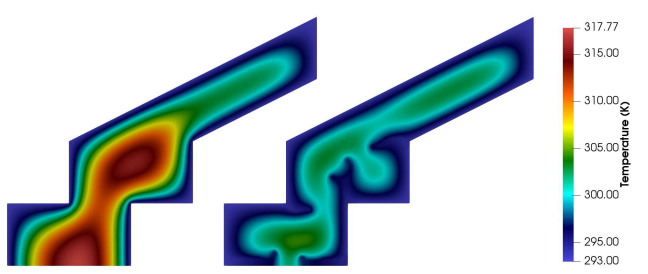

(d) Temperature in homogeneous and optimised tanks at $t_{\text {fin }}$

\section{\begin{tabular}{l|l|l}
$J_{a}$ Homogenous & $J_{a}$ Optimised & Improvement
\end{tabular} \\ \begin{tabular}{l|l|l}
$1.59 \mathrm{~kg}$ & $1.62 \mathrm{~kg}$ & $\mathbf{1 . 9 4 \%}$
\end{tabular}}

Figure 5.13: Optimised design for a custom axisymmetric domain subjected to forced convection with $h=700 \mathrm{Wm}^{-2} \mathrm{~K}^{-1}$. 


\section{Domain}

Heat Convection: $\quad h_{\Gamma_{W}}=5 \mathrm{Wm}^{-2} \mathrm{~K}^{-1}$

Cold Finger: $\quad T_{\Gamma_{F}}=273 \mathrm{~K}$

\section{Cycle}

Custom Axisymmetric Tank

Time Length: $\quad t_{f i n, a}=3600$ s, Fig. (3.3)

(Table 5.3)

$$
\begin{aligned}
\text { Optimisation } & \\
\text { Material Model: } & \epsilon=0.02, \mathrm{Eq} .(3.13) \\
& p=[20,100], \Delta p=10 \\
\text { Iterations per Step: } & i t_{\max }=10 \\
\text { Tolerance: } & \omega_{\min }=1.0 \times 10-6 \\
\text { Filter Radius: } & z=5 \times 10^{-3} \mathrm{~m}, \text { Eq.(4.44) }
\end{aligned}
$$

Table 5.15: Optimisation definitions for a custom axisymmetric domain subjected to natural convection and connected to a cold finger. 

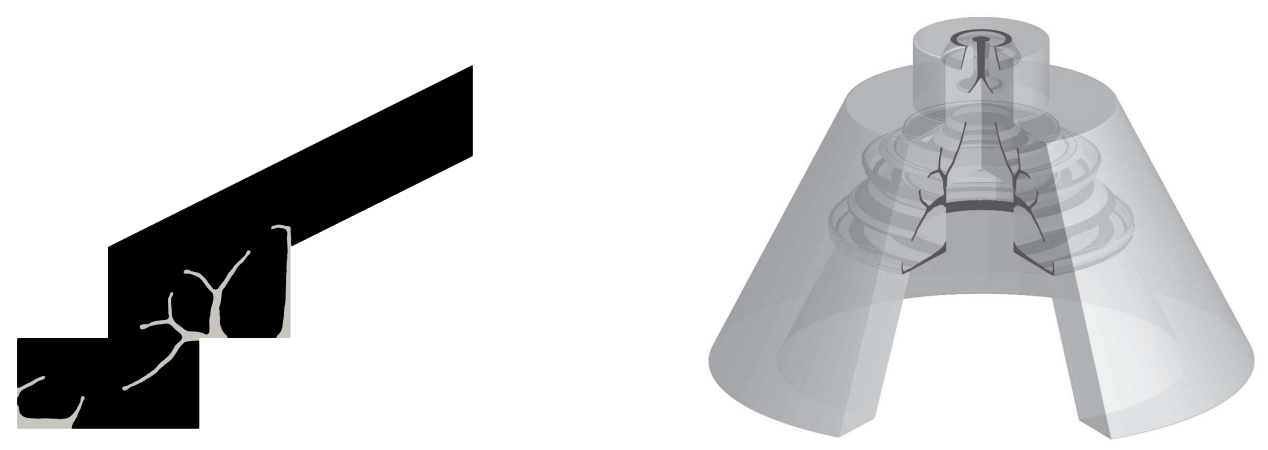

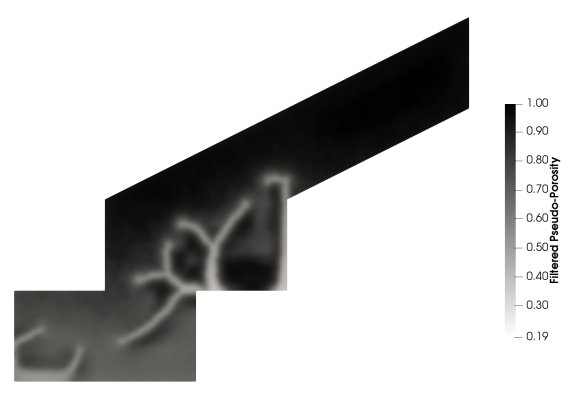

(a) Pseudo-porosity distribution

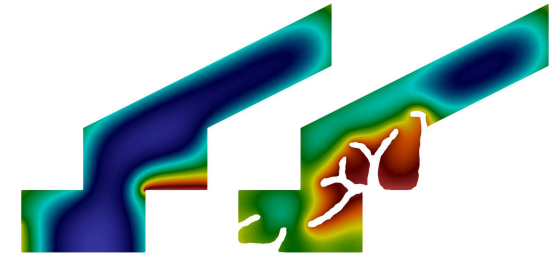

(c) Total gas in homogeneous and optimised tanks at $t_{\text {fin }}$

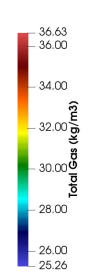

(d) Temperature in
mised tanks at $t_{\text {fin }}$

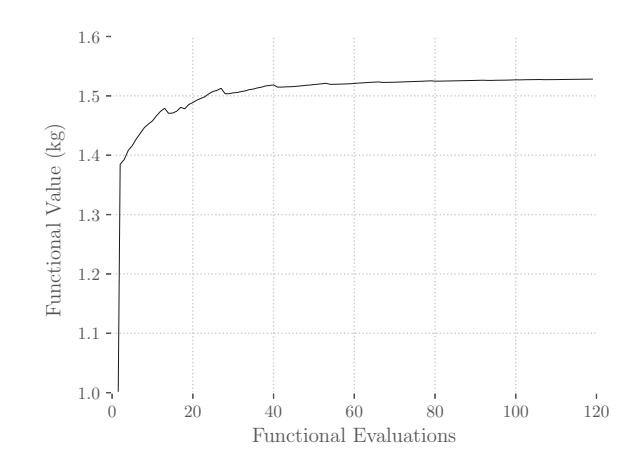

(b) Functional convergence

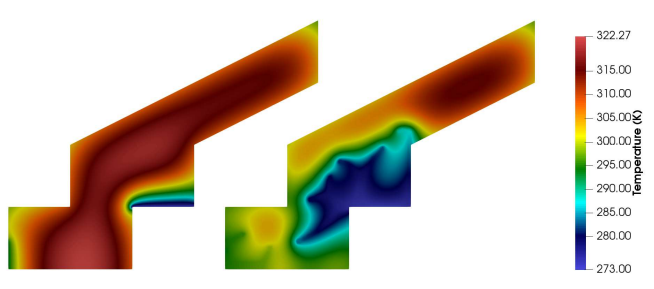

(d) Temperature in homogeneous and optimised tanks at $t_{\text {fin }}$

$$
\begin{array}{c|c|c}
J_{a} \text { Homogenous } & J_{a} \text { Optimised } & \text { Improvement } \\
1.42 \mathrm{~kg} & 1.52 \mathrm{~kg} & \mathbf{7 . 5 1 \%}
\end{array}
$$

Figure 5.14: Optimised design for a cylindrical domain with forced convection. 


\subsubsection{Sensitivity to Shape Complexity}

The cases tested in the previous Section are repeated for a cylindrical tank, in order to identify the contribution of the external shape complexity to the optimisation. Forced convection is imposed on the cylindrical tank, as defined in Table 5.16. As seen in Figure 5.15 , such convection urges the appearance of metallic fins on the wall opposite to the inlet. The main reason for such fins appearing there is the adiabatic condition imposed on the bottom line due to the axisymmetry. In the temperature distribution shown for the homogeneous case in Figure 5.15d it can be seen that the axisymmetry makes the gradient along the bottom line near the walls to be the steepest.

By shortening the cycle defined in Table 5.16 to $3600 \mathrm{~s}$, the volume occupied by the fins are slight smaller, although maintaining the roughly the same layout. The fins on the middle of the vertical walls are longer because the gradient of temperature there is approximately the same of the one observed on the bottom line for a short cycle, as shown in Figure 5.16d.

Keeping natural convection on the short cycle and imposing a cold region on $\Gamma_{F}$ results in the topology shown in Figure 5.17. The presence of such condition stimulate the grow of fins from $\Gamma_{F}$ and leads to a net gain in the mass of gas admitted of $2.47 \%$.

$$
\begin{aligned}
\begin{array}{r}
\text { Domain } \\
\text { Heat Convection: }
\end{array} & h_{\left(\Gamma_{W} \cup \Gamma_{F}\right)}=700 \mathrm{~W} \mathrm{~m}^{-2} \mathrm{~K}^{-1} \\
\text { Cycle } & \\
\text { Time Length: } & t_{f i n, a}=10800 \mathrm{~s} \text {, Fig. }(3.3) \\
\text { Optimisation } & \\
\text { Material Model: } & \epsilon=0.02, \mathrm{Eq} .(3.13) \\
& p=[20,100], \Delta p=10 \\
\text { Iterations per Step: } & i t_{\max }=10 \\
\text { Tolerance: } & \omega_{\min }=1.0 \times 10-6 \\
\text { Filter Radius: } & z=5 \times 10^{-3} \mathrm{~m}, \mathrm{Eq} .(4.44)
\end{aligned}
$$$$
\text { Cylindrical Tank }
$$$$
\text { (Table 5.2) }
$$

Table 5.16: Optimisation definitions for a cylindrical domain with forced convection in 10800 s filling cycle. 


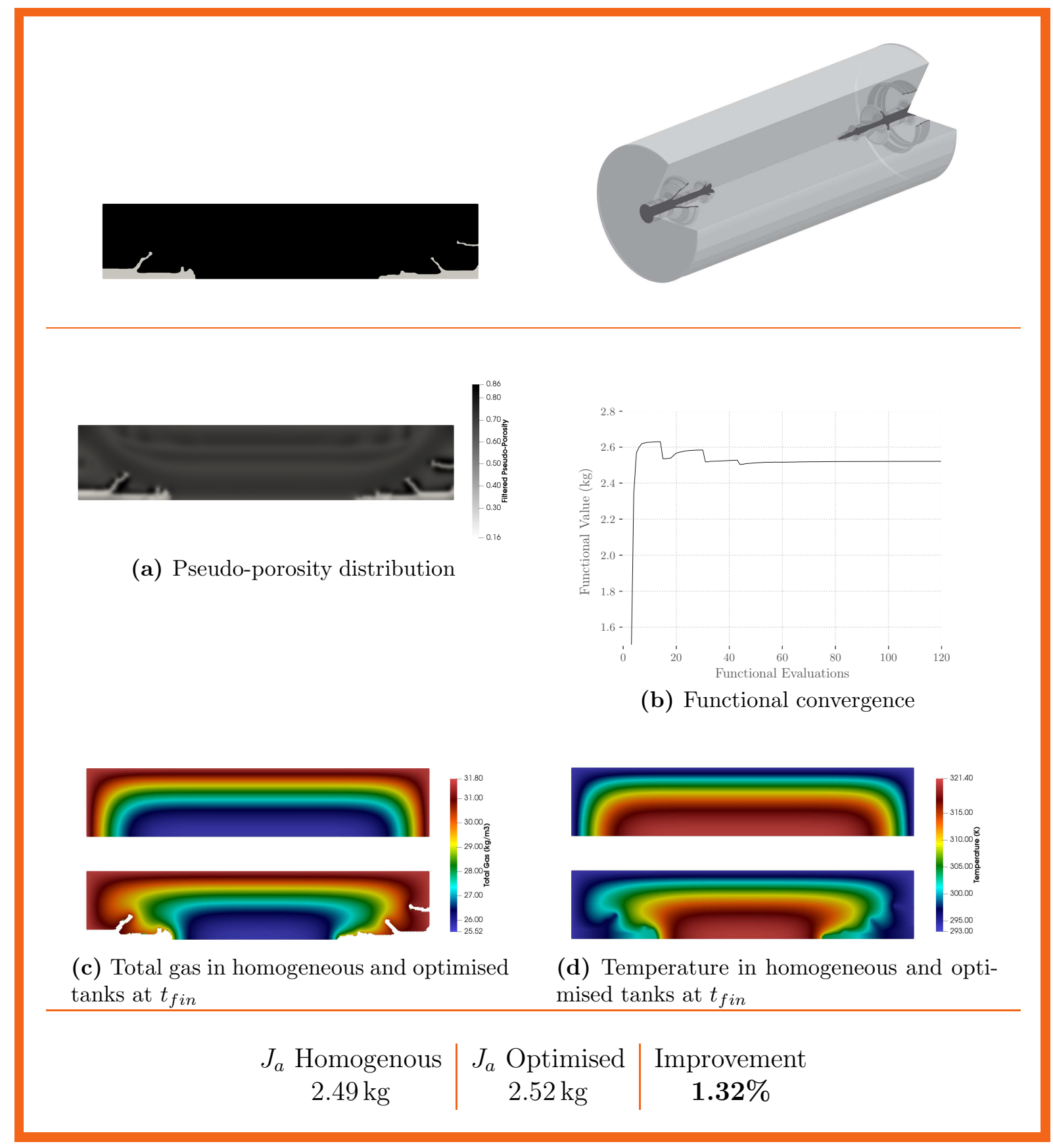

Figure 5.15: Optimised design for a cylindrical domain with forced convection in $10800 \mathrm{~s}$ filling cycle. 


\section{Domain \\ Heat Convection: $\quad h_{\left(\Gamma_{W} \cup \Gamma_{F}\right)}=700 \mathrm{~W} \mathrm{~m}^{-2} \mathrm{~K}^{-1}$}

Cycle

Time Length: $\quad t_{f i n, a}=3600 \mathrm{~s}$, Fig. (3.3)

Cylindrical Tank

(Table 5.2)

\section{Optimisation}

Material Model:

Iterations per Step:

Tolerance:

Filter Radius: $z=5 \times 10^{-3} \mathrm{~m}$, Eq.(4.44)

Table 5.17: Optimisation definitions for a cylindrical domain with forced convection in 3600 s filling cycle.

\section{Domain \\ Heat Convection: $\quad h_{\Gamma_{W}}=5 \mathrm{Wm}^{-2} \mathrm{~K}^{-1}$ \\ Cold Finger: $T_{\Gamma_{F}}=273 \mathrm{~K}$}

\section{Cycle}

Time Length: $\quad t_{f i n, a}=3600 \mathrm{~s}$, Fig. (3.3)

Cylindrical Tank

(Table 5.2)

Table 5.18: Optimisation definitions for a cylindrical domain subjected to natural convection and connected to a cold finger. 


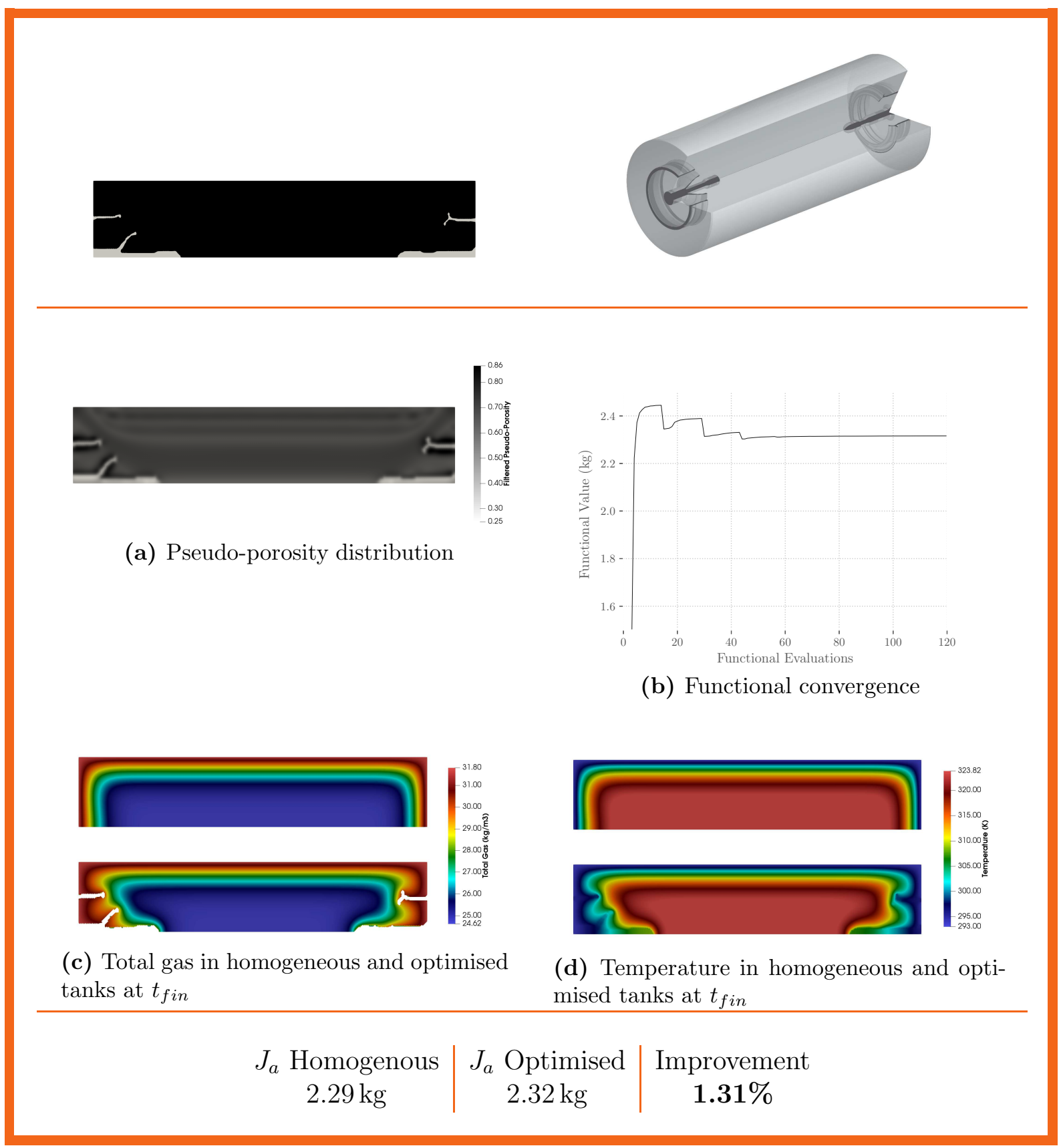

Figure 5.16: Optimised design for a cylindrical domain with forced convection in $3600 \mathrm{~s}$ filling cycle. 


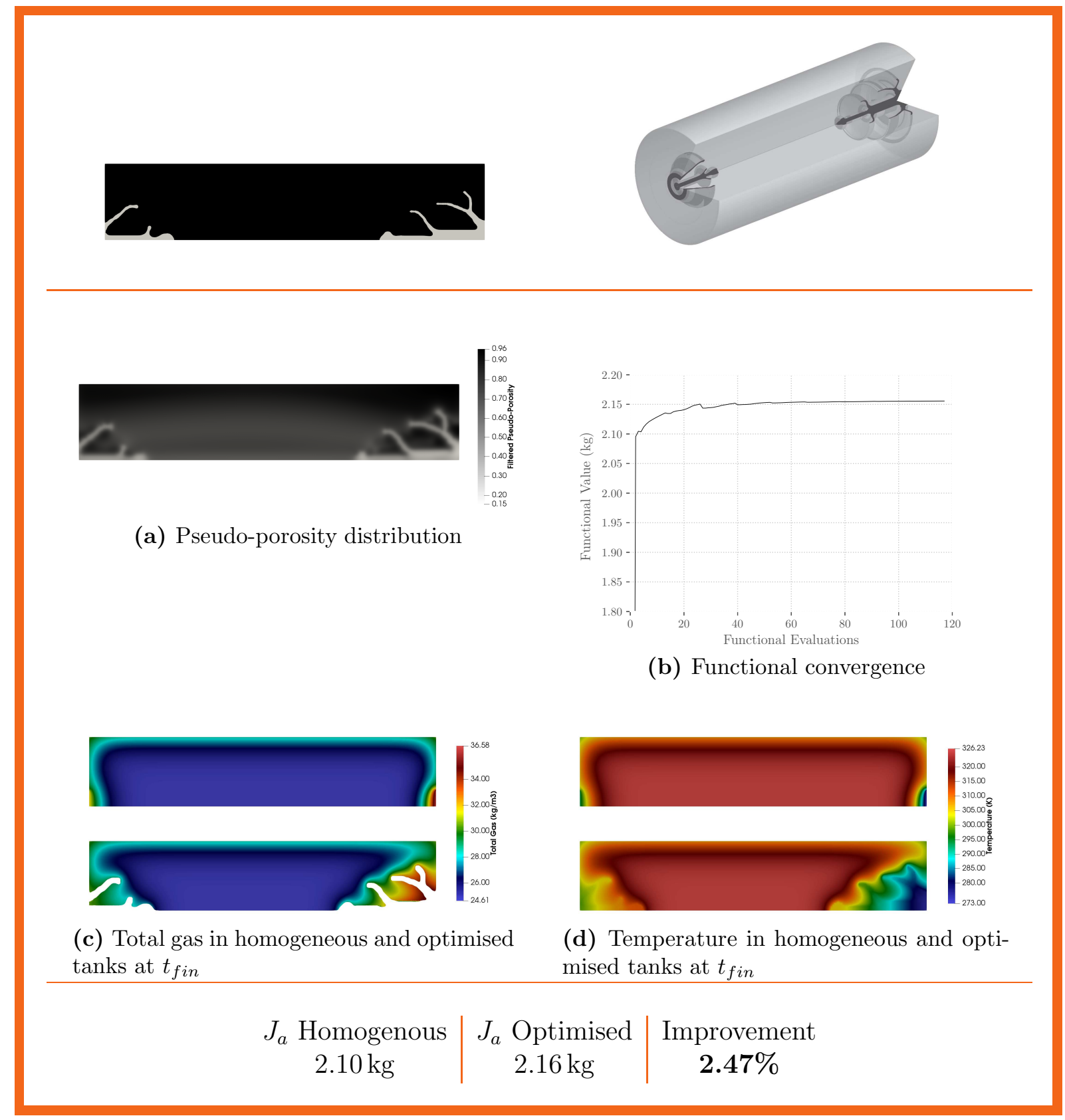

Figure 5.17: Optimised design for a cylindrical domain with natural convection and cold finger. 


\subsubsection{Sensitivity to Tank Dimensions}

Prismatic tanks with different sizes of cross section are optimised aiming to evaluate the sensitivity of the method to tank dimensions. The optimisation of the narrow tanks defined in Table 5.20 is performed with the proportional reduction of the Helmholtz filter radius. In the resulting topology presented in Figure 5.18, it is seen a smaller penetration of the fins towards the center of the domain. However, the distance between the tips of opposite fins are barely the same when compared to the optimised topology for the analogous $0.25 \mathrm{~m}$ tank presented in Figure 5.11. This distance is determined by Activated Carbon heat conduction. Figure 5.18c shows very small gradients in the distribution of gas density when compared to the $0.25 \mathrm{~m}$ reference. While in the homogeneous reference the minimal density observed is $24.74 \mathrm{~kg}$, here it is $29.47 \mathrm{~kg}$, making the loss of adsorbent volume to fins less advantageous. In fact, the improvement of gas intake is much smaller in the small domain: $0.39 \mathrm{~kg}$.

Table 5.19 defines a large prismatic tank, with the proportional increase of the Helmholtz radius filter. The resulting topology shown in Figure 5.19 presents many more fins than in the smaller tanks. The pseudo-porosity distribution in Figure 5.19a shows the formation of perfect squared region on the middle of the domain where the value of the design variables is very near to $e=1.0$. The grey margin presents a thickness of approximately $0.12 \mathrm{~m}$, suggesting that beyond this point the loss in volume for fins is not compensatory. It can be seen in Figure 5.19b that many pseudo-porosity distributions were rejected by L-BFGS-B while the penalisation was weak, evolving to a smooth convergence for higher penalisation. Figure 5.19c makes clear that little improvement could be achieved in the central tank region. The focus of the optimisation was to adsorb more gas near the walls, leaving the central region almost unchanged. The improvement in the mass of gas admitted is roughly equivalent to the gain presented for the $0.25 \mathrm{~m}$ reference: $5.54 \%$.

\subsubsection{Sensitivity to Filling Pressure}

The optimisation of ANG tanks are highly dependent on its filling pressure. Aiming to demonstrate the topology changes caused by the pressure increase, the problem described is Table 5.13 is used as reference. By increasing the filling pressure from $1.00 \mathrm{MPa}$ to 3.00 MPa, the topology shown in Figure 5.20 is obtained. In contrast to the resulting topology for $1.00 \mathrm{MPa}$, it can be seen that the higher gas flow rate is enough to cool down a large area around the inlet. Therefore, fins connected to the inlet region are less interesting in this case, given that a large volume of cold adsorbent would be lost. Fins 


$$
\begin{aligned}
\text { Domain } & \\
\text { Dimensions: } & L=0.10 \mathrm{~m} \\
\text { Heat Convection: } & h_{\Gamma_{W}}=100 \mathrm{Wm}^{-2} \mathrm{~K}^{-1}
\end{aligned}
$$

\section{Cycle}

Time Length: $\quad t_{\text {fin }, a}=10800 \mathrm{~s}$, Fig. (3.3)

Prismatic Tank

(Table 5.1)

\section{Optimisation}

Material Model:

Iterations per Step:

Tolerance:

Filter Radius: $\epsilon=0.02$, Eq. $(3.13)$

$p=[20,100], \Delta p=10$

$i t_{\max }=10$

$\omega_{\min }=1.0 \times 10-6$

$z=2 \times 10^{-3} \mathrm{~m}$, Eq.(4.44)

Table 5.19: Optimisation definitions for a small prismatic domain subjected to forced convection.

$$
\begin{aligned}
\text { Domain } & \\
\text { Dimensions: } & L=0.40 \mathrm{~m} \\
\text { Heat Convection: } & h_{\Gamma_{W}}=100 \mathrm{Wm}^{-2} \mathrm{~K}^{-1}
\end{aligned}
$$

\section{Cycle}

Time Length: $\quad t_{f i n, a}=10800 \mathrm{~s}$, Fig. (3.3)

Prismatic Tank

(Table 5.1)

\section{Optimisation}

Material Model:

Iterations per Step:

Tolerance:

Filter Radius: $\epsilon=0.02$, Eq. $(3.13)$

$p=[20,100], \Delta p=10$

$i t_{\max }=10$

$\omega_{\text {min }}=1.0 \times 10-6$

$z=8 \times 10^{-3} \mathrm{~m}$, Eq.(4.44)

Table 5.20: Optimisation definitions for a small prismatic domain subjected to forced convection.

are then place on the nearest concave corner, producing a slight temperature reduction in the region, which results in an improvement of $0.23 \%$ on the intake mass of gas. Such gain is much smaller than the one obtained for the reference case, demonstrating that the addition of fins are more suitable on the design of low pressure ANG tanks.

By increasing the filling pressure to $5.00 \mathrm{MPa}$, as defined in Table 5.22, the fins are 


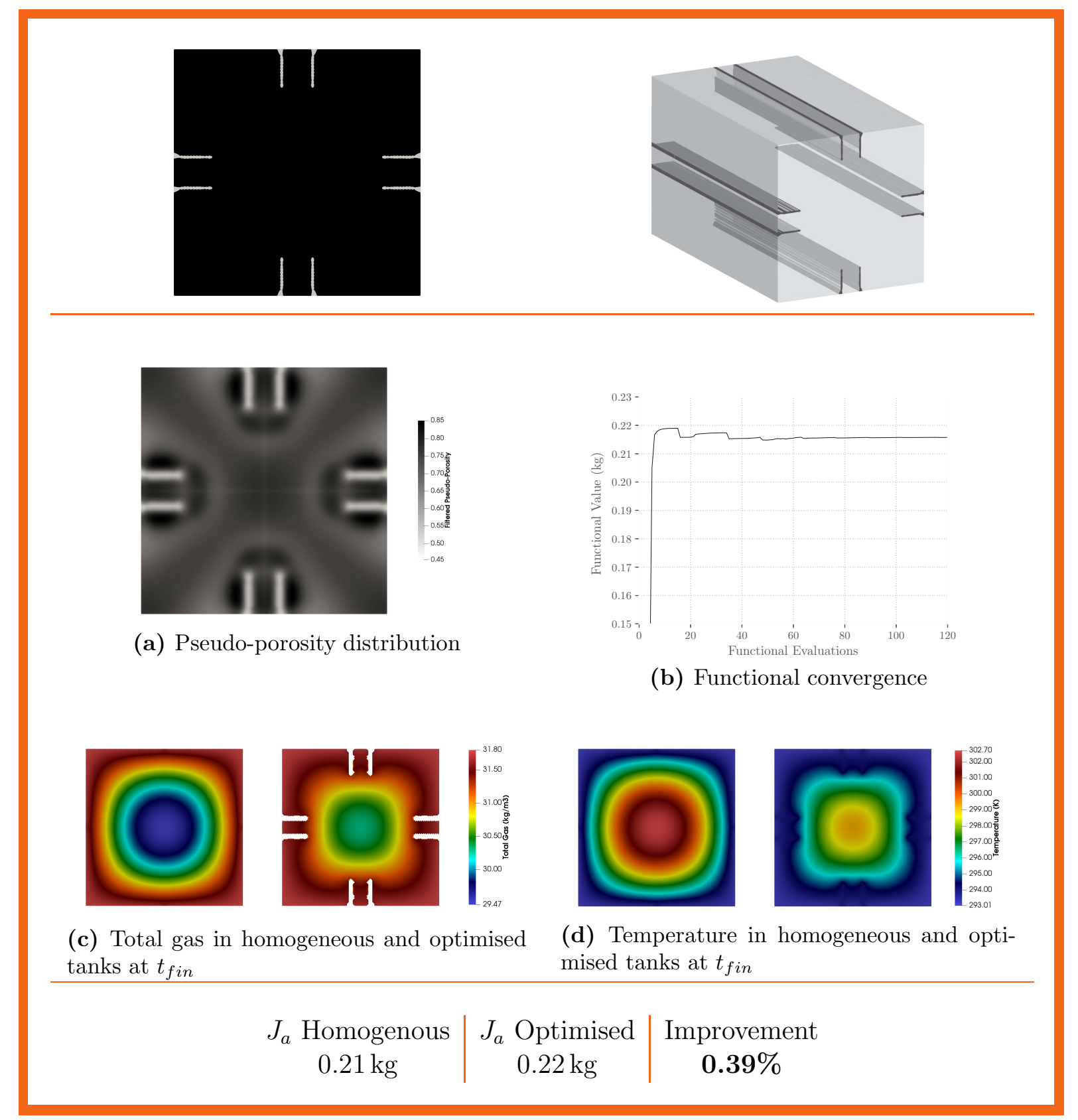

Figure 5.18: Optimised design for a small prismatic domain subjected to forced convection.

placed farther than the inlet region, as shown in Figure 5.21. This shift is explained by extending the points listed on the $3.00 \mathrm{MPa}$ case. The 5.00 MPa pressure intensified the cooling effect produced near the inlet region, as seen in Figure 5.21d. Interestingly, the improvement achieved here is slightly higher than in the $3.00 \mathrm{MPa}$ case: $0.58 \%$. Such improvement is mainly due to the creation of a fin on the next concave corner, which was able to reduce the maximum temperature inside the optimised tank to around $320 \mathrm{~K}$. 


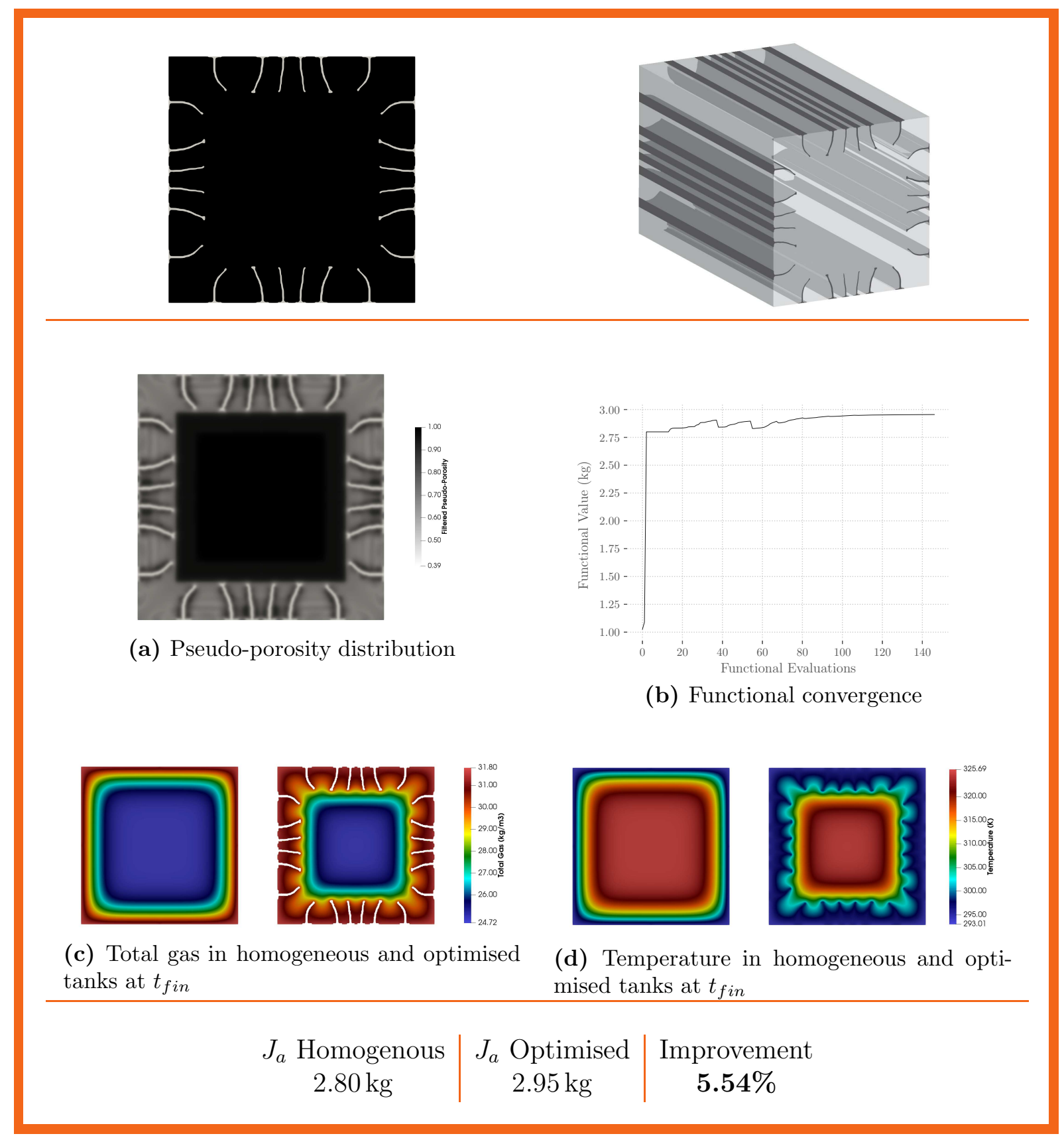

Figure 5.19: Optimised design for a large prismatic domain subjected to forced convection. 


\section{Domain}

Heat Convection: $\quad h_{\left(\Gamma_{W} \cup \Gamma_{F}\right)}=100 \mathrm{~W} \mathrm{~m}^{-2} \mathrm{~K}^{-1}$

\section{Cycle}

Time Length: $\quad t_{f i n, a}=3600 \mathrm{~s}$, Fig. $(3.3)$

Custom Axisymmetric Tank Final Pressure: $\quad P_{l e t}=3.00 \mathrm{MPa}$

(Table 5.3)

$$
\begin{aligned}
\text { Optimisation } & \\
\text { Material Model: } & \epsilon=0.02, \mathrm{Eq} .(3.13) \\
& p=[20,100], \Delta p=10 \\
\text { Iterations per Step: } & i t_{\max }=10 \\
\text { Tolerance: } & \omega_{\min }=1.0 \times 10-6 \\
\text { Filter Radius: } & z=5 \times 10^{-3} \mathrm{~m}, \text { Eq.(4.44) }
\end{aligned}
$$

Table 5.21: Optimisation definitions for a custom axisymmetric domain subjected to $3.00 \mathrm{MPa}$ filling pressure and forced convection with $h=100 \mathrm{Wm}^{-2} \mathrm{~K}^{-1}$.

\section{Domain}

Heat Convection: $\quad h_{\left(\Gamma_{W} \cup \Gamma_{F}\right)}=100 \mathrm{~W} \mathrm{~m}^{-2} \mathrm{~K}^{-1}$

\section{Cycle}

Time Length: $t_{f i n, a}=3600 \mathrm{~s}$, Fig. (3.3)

Custom Axisymmetric Tank

$$
\text { Final Pressure: } P_{l e t}=5.00 \mathrm{MPa}
$$

(Table 5.3)

$$
\begin{aligned}
\text { Optimisation } & \\
\text { Material Model: } & \epsilon=0.02, \text { Eq. }(3.13) \\
& p=[20,100], \Delta p=10 \\
\text { Iterations per Step: } & i t_{\max }=10 \\
\text { Tolerance: } & \omega_{\min }=1.0 \times 10-6 \\
\text { Filter Radius: } & z=5 \times 10^{-3} \mathrm{~m}, \text { Eq. }(4.44)
\end{aligned}
$$

Table 5.22: Optimisation definitions for a custom axisymmetric domain subjected to $5.00 \mathrm{MPa}$ filling pressure and forced convection with $h=100 \mathrm{Wm}^{-2} \mathrm{~K}^{-1}$. 


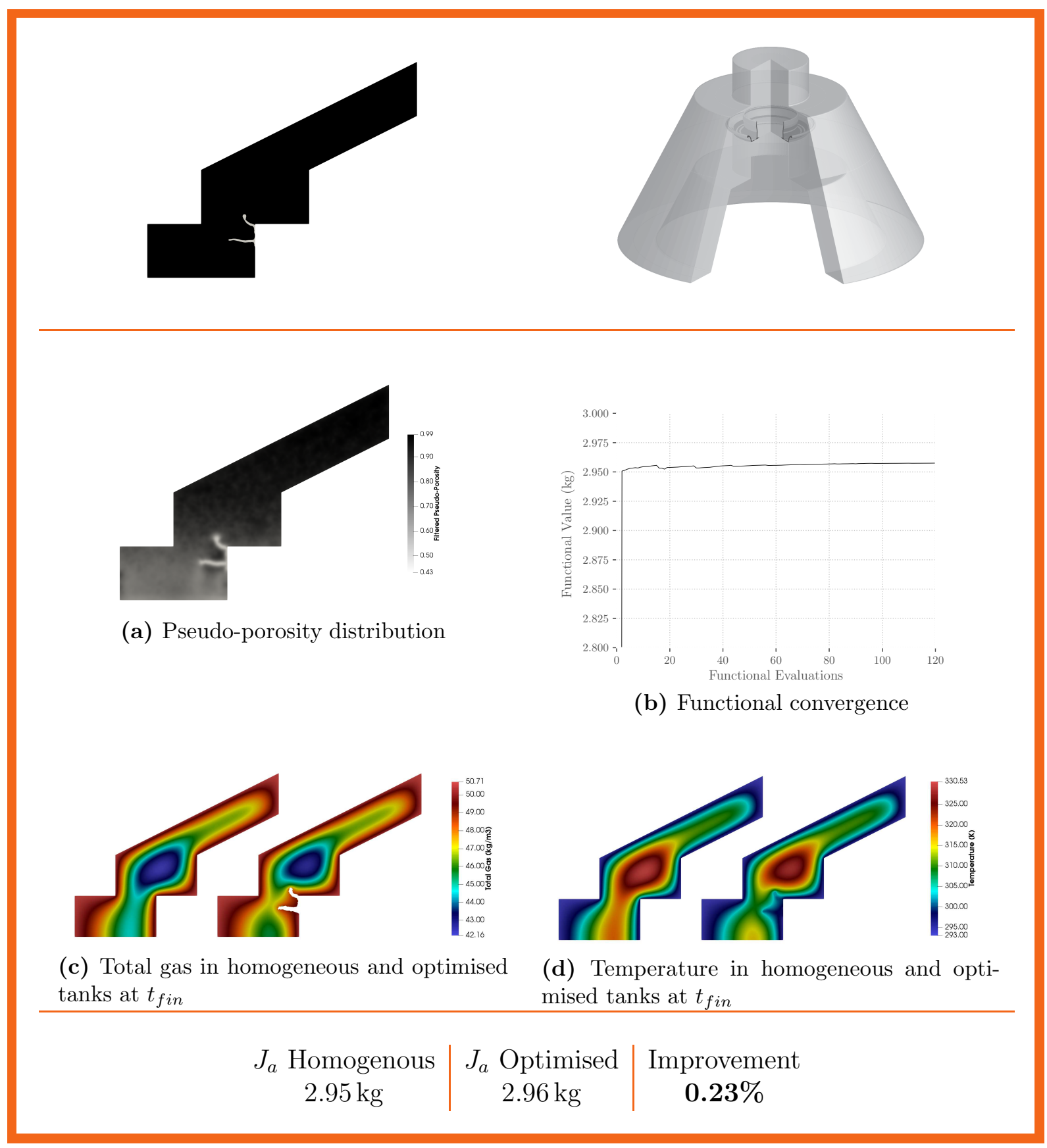

Figure 5.20: Optimised design for a custom axisymmetric domain subjected to $3 \mathrm{MPa}$ filling pressure and forced convection with $h=100 \mathrm{Wm}^{-2} \mathrm{~K}^{-1}$. 

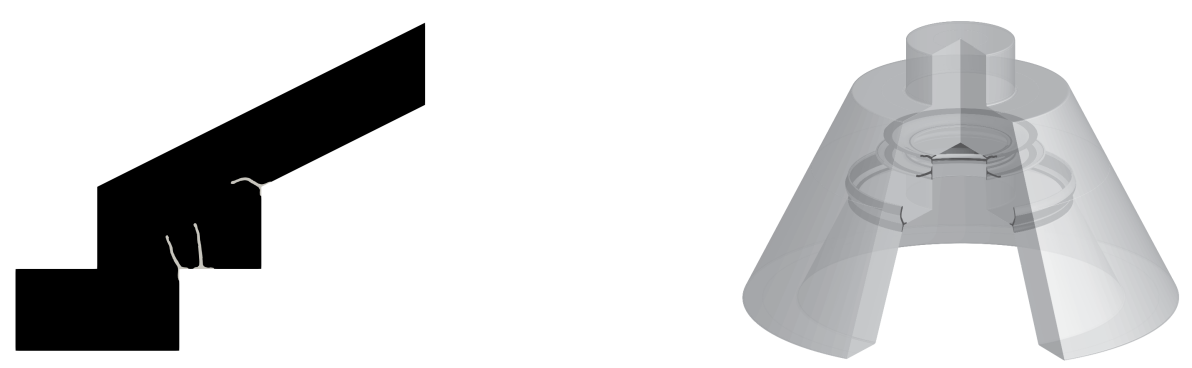

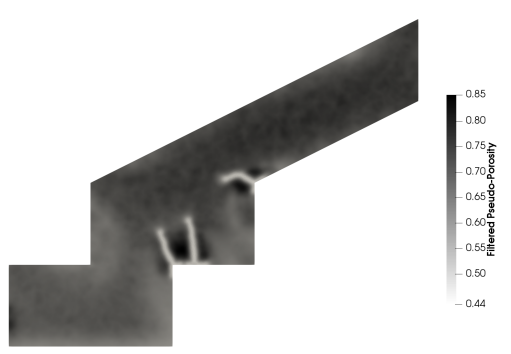

(a) Pseudo-porosity distribution

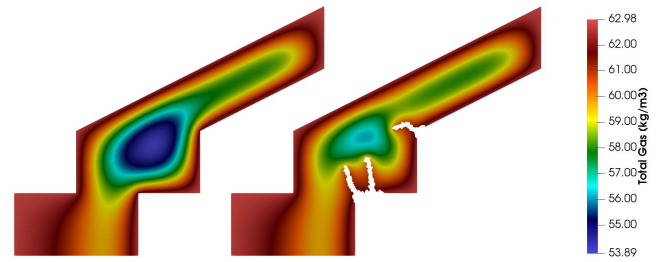

(c) Total gas in homogeneous and optimised tanks at $t_{\text {fin }}$

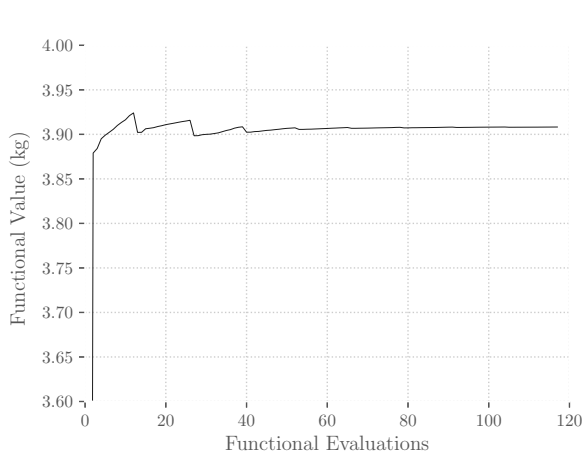

(b) Functional convergence

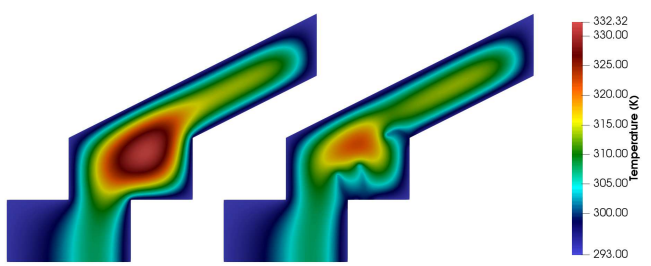

(d) Temperature in homogeneous and optimised tanks at $t_{\text {fin }}$

$$
\begin{array}{c|c|c}
J_{a} \text { Homogenous } & J_{a} \text { Optimised } & \text { Improvement } \\
3.89 \mathrm{~kg} & 3.91 \mathrm{~kg} & \mathbf{0 . 5 8 \%}
\end{array}
$$

Figure 5.21: Optimised design for a custom axisymmetric domain subjected to $5 \mathrm{MPa}$ filling pressure and forced convection with $h=100 \mathrm{Wm}^{-2} \mathrm{~K}^{-1}$. 


\section{Conclusions}

The main goal of the research presented in this work was to formulate and evaluate the use of topology optimisation for designing adsorption systems. Among the several applications of such systems, Adsorbed Natural Gas (ANG) tanks have been chosen to be the focus of this work. Accordingly, a comprehensive model for ANG tanks is presented, including the kinetics of adsorption and the variation of adsorbent properties in function to the porosities. The solution of the resulting set of PDEs governing the adsorption problem is obtained via the Finite Element Method (FEM). The procedure to assemble the FEM matrices from the PDEs weak form is described in detail. The concept of including nonadsorbent materials inside ANG tanks aiming to improve their thermal management is then introduced. The optimisation of the distribution of adsorbent and non-adsorbent materials inside the tank is approached via topology optimisation. The implementation of this method is based on the calculation of the sensitivities via the adjoint method for transient systems, which is comprehensively presented. Resulting optimised topologies are presented for ANG tanks applied to on-demand consumption. The optimisation of tanks with different shapes is tackled and the impact of parameters such as material properties, filling pressure, cycle duration and tank dimensions are studied.

A major scientific contribution presented in this work is the derivation of the full formulation of a gradient-based PDE-constrained optimisation of adsorption systems. Such derivation includes the statement of the weak and discrete formulation of the adsorption problem for FEM implementation and the derivation of the adjoint problem from the augmented Lagrangian form of the functional of interest. Additionally, a material model based on hyperbolic tangents (OHT) is introduced. Such model has shown to be more efficient than traditional ones for the design of biphasic structures. It is due to its novel penalisation approach combined with offsets.

Several ANG tanks optimised according to the distribution of cooling material across 
the adsorbent bed are presented in this work. Designs include axisymmetric and prismatic tanks subjected to natural convection and active thermal boundary conditions. By the results presented, it is concluded that small improvement is achievable by the addition of metallic fins in tanks subjected to natural convection. For the cycles and domain sizes considered, tanks subjected to natural convection presented insufficient improvement in order to justify the cost of including regions constituted by non-adsorbent material across the adsorbent bed. Similarly, small improvement is observed in high pressure tanks. The relative improvement achievable in high pressure tanks is smaller than the ones working at low pressure. It suggests that the design concept adopted by this work is more suitable for low pressure ANG tanks.

In contrast, the insertion of non-adsorbent regions in ANG tanks is very effective when associated with cold regions. It is due to the fact that materials with high conductivity depend on meaningful temperature gradients in order to aid heat transfer across the tank. Such fact is reinforced by the resulting topologies presenting metallic regions around tanks concave corners, where the temperature gradients are higher. Also, materials presenting high heat capacity constitute a suitable solution for passive thermal management. In opposition to phases presenting typical metallic properties, materials with high heat capacity are not dependent on the existence of cold regions in the domain. Results have shown that islands constituted by materials presenting high heat capacity act as a heat sink, creating a zone of influence in the temperature field.

Altogether, adsorbent's low conductivity can represent a limiting issue. In fast adsorption cycles, any optimisation attempt is hindered by the extremely low conductivity of adsorbent materials. It is demonstrated by the reduction of the zone of influence of regions constituted by high capacity materials as the cycles considered gets shorter. Therefore, the design concept presented in this work is complementary to further development of adsorbent materials.

This thesis provides the basis for several future work. The formulation presented here is easily extended to storage and transport applications, by including the desorption cycle in the objective function. Additionally, since results have shown that complex-shaped tanks present better performance than traditional ones. Therefore, it is promising to complement the optimisation of the adsorbent bed with the optimisation of the external shape, by either shape or topology optimisation. Furthermore, given the promising results obtained for non-adsorbent material with high heat capacity, the use of Phase Change Materials (PCMs) is very promising for any application. It consists of including an additional governing equation in order to model the phase change kinetics, leading to new 
source terms in the energy balance.

Due to the numerical focus of this work, manufacturing issues have been addressed marginally only. Yet, the literature review in this matter indicated relative ease for conforming adsorbent beds in any desired geometry by pouring and compacting a slurry constituted by binder-coated carbon particles inside tank segments. Manufacturing complexity depend mainly on the construction of non-adsorbent parts, which can be done via traditional methods such as machining and casting, in the case of metals. However, despite such preliminary indications, further research on this topics is necessary.

Finally, the work presented here is naturally extendible to any other adsorption application. Fluid separation, refrigeration and Carbon Capture and Storage (CCS) are some of the possible applications for adsorption systems that could be explored based on this research. 


\section{References}

ALKAN, M.; DEMIRBAS, O.; DOGAN, M. Adsorption kinetics and thermodynamics of an anionic dye onto sepiolite. Microporous and Mesoporous Materials, v. 101, n. 3, p. 388-396, abr. 2007. ISSN 13871811.

ALNÆS, M. S. et al. Unified form language. ACM Transactions on Mathematical Software, v. 40, n. 2, p. 1-37, nov. 2014. ISSN 00983500.

ANDREASEN, C. S.; SIGMUND, O. Topology optimization of fluid-structureinteraction problems in poroelasticity. Computer Methods in Applied Mechanics and Engineering, Elsevier B.V., v. 258, p. 55-62, maio 2013. ISSN 00457825.

ANGP. Low-Pressure is the Future of Natural Gas Vehicles. 2017. Disponível em: $\langle$ http://www.angpinc.com/phangv/〉.

ANISZEWSKI, W.; MENARD, T.; MAREK, M. Volume of fluid (vof) type advection methods in two-phase flow: A comparative study. Computers $\&$ Fluids, Elsevier Ltd, v. 97, p. 52-73, 2014. ISSN 00457930.

BENDSØE, M. P.; SIGMUND, O. Material interpolation schemes in topology optimization. Archive of Applied Mechanics, v. 69, n. 9-10, p. 635-654, 1999. ISSN 0939-1533.

BILOE, S.; GOETZ, V.; GUILLOT, A. Optimal design of an activated carbon for an adsorbed natural gas storage system. Carbon, v. 40, n. 8, p. 1295-1308, 2002. ISSN 00086223.

BIRD, R. B.; STEWART, W. B.; LIGHTFOOT, E. N. Transport Phenomena. 2nd. ed. [S.l.: s.n.], 2007. ISBN 978-0470115398.

BLANCO, M. V. et al. Hydrogen sorption kinetics of la-ni-sn storage alloys. International Journal of Hydrogen Energy, Elsevier Ltd, v. 39, n. 11, p. 5858-5867, 2014. ISSN 0360-3199.

BORRVALL, T.; PETERSSON, J. Topology optimization of fluids in stokes flow. International Journal for Numerical Methods in Fluids, v. 41, n. 1, p. 77-107, 2003. ISSN 02712091.

BOSE, T. K.; CHAHINE, R.; ST-ARNAUD, J. M. High-density adsorbent and method of producing same. US Patent 4999330A, 1991.

BRUNS, T. E. Topology optimization of convection-dominated, steady-state heat transfer problems. International Journal of Heat and Mass Transfer, v. 50, n. 15-16, p. 2859-2873, 2007. ISSN 00179310.

BYRD, R. et al. A limited memory algorithm for bound constrained optimization. SIAM Journal on Scientific Computing, v. 16, n. 5, p. 1190-1208, 1995. 
CARMAN, P. C. Fluid flow through granular beds. Chemical Engineering Research and Design, Institution of Chemical Engineers, v. 75, p. S32-S48, 1937. ISSN 02638762.

CAVALCANTE, C. L. Industrial adsorption separation processes: Fundamentals, modeling and applications. Latin American Applied Research, v. 30, n. 4, p. 357-364, 2000. ISSN 03270793.

CHAO, T. Y.; CHOW, W. K.; KONG, H. A review on the applications of finite element method to heat transfer and fluid flow. International Journal on Architectural Science, v. 3, n. 1, p. 1-19, 2002.

CHARTON, F.; NICOUD, R. M. Complete design of a simulated moving bed. Journal of Chromatography A, v. 702, n. 1-2, p. 97-112, maio 1995. ISSN 00219673.

CHRISTMANN, K. Introduction to Surface Physical Chemistry. [S.l.]: Springer Berlin Heidelberg, 1991. ISBN 0195105893.

DAHL, J.; JENSEN, J. S.; SIGMUND, O. Topology optimization for transient wave propagation problems in one dimension : Ddesign of filters and pulse modulators. Structural and Multidisciplinary Optimization, v. 36, n. 6, p. 585-595, 2008. ISSN $1615147 \mathrm{X}$.

DIJK, N. P. et al. Level-set methods for structural topology optimization: A review. Structural and Multidisciplinary Optimization, v. 48, n. 3, p. 437-472, 2013. ISSN $1615147 \mathrm{X}$.

ELROD, M. J. Greenhouse warming potentials from the infrared spectroscopy of atmospheric gases. Journal of Chemical Education, v. 76, n. 12, p. 1702, 1999.

FUNKE, S. W. The automation of PDE-constrained optimisation and its applications. Tese (Doutorado) — Imperial College London, 2012.

GERSBORG-HANSEN, A.; SIGMUND, O.; HABER, R. B. Topology optimization of channel flow problems. Structural and Multidisciplinary Optimization, v. 30, n. 3, p. 181-192, jun. 2005. ISSN 1615147X.

GILES, C. H. A general treatment and classification of the solute adsorption isotherm. Journal of Colloid and Interface Science, v. 47, n. 3, 1974.

GUEST, J. K.; PREVOST, J. H. Topology optimization of creeping fluid flows using a darcy-stokes finite element. International Journal for Numerical Methods in Engineering, v. 66, n. 3, p. 461-484, abr. 2006. ISSN 00295981.

HALL, J. et al. A volumetric geometry and topology parameterisation for fluids-based optimisation. Computers and Fluids, Elsevier Ltd, v. 148, p. 137-156, 2017. ISSN 00457930 .

HASCOET, L.; DAUVERGNE, B. Adjoints of large simulation codes through automatic differentiation. European Journal of Computational ..., p. 1-24, 2008. ISSN 17797179.

HIRATA, S. C. et al. Modeling and hybrid simulation of slow discharge process of adsorbed methane tanks. International Journal of Thermal Sciences, Elsevier Masson SAS, v. 48, n. 6, p. 1176-1183, jun. 2009. ISSN 12900729. 
INGEVITY. Turning complex challenges into powerful possibilities. 2017. Disponível em: 〈http://www.ingevity.com/markets/adsorbed-natural-gas/ $\rangle$.

JONES, E.; OLIPHANT, T.; PETERSON, P. SciPy: Open Source Scientific Tools for Python. 2011. Disponível em: 〈http://www.scipy.org $\rangle$.

JUNTUNEN, M.; STENBERG, R. General boundary conditions. v. 78, n. 267, p. 1353-1374, 2012.

KAIST. Box-shaped Pressure Vessel for LNG Developed by KAIST Research Team. 2014. Disponível em: 〈http://www.kaist.edu〉.

KARNIADAKIS, G. et al. Spectral/hp Element Methods for Computational Fluid Dynamics: Second Edition. [S.1.]: OUP Oxford, 2005. (Numerical Mathematics and Scie). ISBN 9780198528692.

KIRBY, R. C. Algorithm 839. ACM Transactions on Mathematical Software, v. 30, n. 4, p. 502-516, 2004. ISSN 00983500.

KOGA, A. A. et al. Development of heat sink device by using topology optimization. International Journal of Heat and Mass Transfer, Elsevier Ltd, v. 64, p. 759-772, set. 2013. ISSN 00179310.

KONTOLEONTOS, E. A. et al. Adjoint-based constrained topology optimization for viscous flows, including heat transfer. Engineering Optimization, v. 0273, n. December, p. 1-21, ago. 2012. ISSN 0305-215X.

LARGITTE, L.; PASQUIER, R. A review of the kinetics adsorption models and their application to the adsorption of lead by an activated carbon. Chemical Engineering Research and Design, v. 109, p. 495-504, 2016. ISSN 18766102.

LAZAROV, B. S.; SIGMUND, O. Filters in topology optimization based on helmholtztype differential equations. International Journal for Numerical Methods in Engineering, v. 86, n. 6, p. 765-781, maio 2011. ISSN 00295981.

LIMOUSIN, G. et al. Sorption isotherms: A review on physical bases, modeling and measurement. Applied Geochemistry, v. 22, n. 2, p. 249-275, 2007. ISSN 08832927.

LOGG, A. Automating the finite element method. Archives of Computational Methods in Engineering, v. 14, n. 2, p. 93-138, dez. 2007. ISSN 11343060.

MATSUMORI, T. et al. Topology optimization for fluid-thermal interaction problems under constant input power. Structural and Multidisciplinary Optimization, v. 47, n. 4, p. 571-581, fev. 2013. ISSN 1615147X.

MCBRIDE, M. B. Environmental Chemistry of Soils. Oxford University Press, 1994. ISBN 9780195070118. Disponível em: 〈https://books.google.co.uk/books? $\mathrm{id}=\mathrm{jgZhQgAACAAJ}\rangle$.

MENON, V. C.; KOMARNENI, S. Porous adsorbents for vehicular natural gas storage: a review. Journal of Porous Materials, v. 5, n. 1, p. 43-58, 1998. ISSN 13802224. 
MORRIS, A. M.; ALLEN, C. B.; RENDALL, T. C. S. Cfd-based optimization of aerofoils using radial basis functions for domain element parameterization and mesh deformation. International Journal for Numerical Methods in Fluids, v. 58, n. 8, p. 827-860, nov. 2008. ISSN 02712091.

MOTA, J. P. B.; ESTEVES, I. A. A. C.; ROSTAM-ABADI, M. Dynamic modelling of an adsorption storage tank using a hybrid approach combining computational fluid dynamics and process simulation. Computers and Chemical Engineering, v. 28, n. 11, p. 2421-2431, out. 2004. ISSN 00981354.

MOTA, J. P. B. et al. Charge dynamics of a methane adsorption storage system: Intraparticle diffusional effects. Adsorption, v. 3, n. 2, p. 117-125, 1996. ISSN 0929-5607.

MULJADI, B. P. et al. The impact of porous media heterogeneity on non-darcy flow behaviour from pore-scale simulation. Advances in Water Resources, Elsevier Ltd., v. 95, p. 329-340, 2016. ISSN 03091708.

PALACIOS, F. et al. Stanford university unstructured (su2): An open-source integrated computational environment for multi-physics simulation and design. 51st AIAA Aerospace Sciences Meeting, n. January, p. 1-60, 2013.

PATIHA et al. The langmuir isotherm adsorption equation: The monolayer approach. IOP Conference Series: Materials Science and Engineering, v. 107, p. 012067, 2016. ISSN 1757-8981.

PIGGOTT, M. D. et al. A new computational framework for multi-scale ocean modelling based on adapting unstructured meshes. International Journal for Numerical Methods in Fluids, v. 56, n. 8, p. 1003-1015, mar. 2008. ISSN 02712091.

QIU, H. et al. Critical review in adsorption kinetic models. Journal of Zhejiang University SCIENCE A, v. 10, n. 5, p. 716-724, maio 2009. ISSN 1673-565X.

QUINN, D. F.; MACDONALD, J. A.; SOSIN, K. Microporous carbons as adsorbents for methane storage. 207th ACS national meeting, v. 39, p. 1304, 121994.

RAHMAN, K. A. et al. Thermal enhancement of charge and discharge cycles for adsorbed natural gas storage. Applied Thermal Engineering, Elsevier Ltd, v. 31, n. 10, p. 1630-1639, jul. 2011. ISSN 13594311.

REDDY, J. N.; GARTLING, D. K. The Finite Element Method in Heat Transfer and Fluid Dynamics, Third Edition. Taylor \& Francis, 2010. (Computational Mechanics and Applied Analysis). ISBN 9781420085983. Disponível em:〈https://books.google.co.uk/ books?id=sv0VKLL5lWUC $\rangle$.

REUTHER, J. J. et al. Constrained multipoint aerodynamic shape optimization using an adjoint formulation and parallel computers, part 1. v. 36, 1999.

SA, L. F. N. et al. Topological derivatives applied to fluid flow channel design optimization problems. Structural and Multidisciplinary Optimization, v. 54, n. 2, p. 249-264, Aug 2016. ISSN 1615-1488. Disponível em: 〈https://doi.org/10.1007/ s00158-016-1399-0〉. 
SAHOO, P. K. et al. Filling characteristics for an activated carbon based adsorbed natural gas storage system. Industrial and Engineering Chemistry Research, v. 50, n. 23, p. 13000-13011, 2011. ISSN 08885885.

SAHOO, S.; RAMGOPAL, M. A simple regression equation for predicting charge characteristics of adsorbed natural gas storage systems. Applied Thermal Engineering, Elsevier Ltd, v. 73, n. 1, p. 1093-1100, 2014. ISSN 13594311.

SANTOS, J. C.; MARCONDES, F.; GURGEL, J. M. Performance analysis of a new tank configuration applied to the natural gas storage systems by adsorption. Applied Thermal Engineering, Elsevier Ltd, v. 29, n. 11-12, p. 2365-2372, ago. 2009. ISSN 13594311.

SEDERBERG, T. W.; PARRY, S. R. Free-form deformation of solid geometric models. ACM SIGGRAPH Computer Graphics, v. 20, n. 4, p. 151-160, 1986. ISSN 00978930.

SIGMUND, O. Design of multiphysics actuators using topology optimization - part i: One material structures. Computer Methods in Applied Mechanics and Engineering, v. 190, n. 49-50, p. 6577-6604, 2001. ISSN 00457825.

SIGMUND, O.; PETERSSON, J. Numerical instabilities in topology optimization: A survey on procedures dealing with checkerboards, mesh-dependencies and local minima. Structural Optimization, v. 16, n. 1, p. 68-75, 1998. ISSN 0934-4373.

SMITH, D. S. et al. Thermal conductivity of porous materials. Journal of Materials Research, v. 28, n. 17, p. 2260-2272, jul. 2013. ISSN 2044-5326.

SOLARNEXT. Adsorption Cooling Technology. [S.l.], 2009. 1 p.

STEIN, W.; JOYNER, D. Sage: System for algebra and geometry experimentation. ... Algebra (SIGSAM Bulletin)(July 2005), v. 39, n. 2, p. 61-64, 2005.

SUN, W.; HUANG, W.; RUSSELL, R. D. Finite difference preconditioning for solving orthogonal collocation equations for boundary value problems. SIAM Journal on Numerical Analysis, v. 33, n. 6, p. 2268-2285, 1996. ISSN 0036-1429.

THACKER, W. E. et al. Modeling variable comparison adsorber performance : concentration and influent of adsorbents. v. 56, n. 3, p. 243-250, 2014.

TOLEDO, M. et al. Use of phase change materials on an adsorbed carbon dioxide storage system. Applied Thermal Engineering, Elsevier Ltd, v. 51, n. 1-2, p. 512-519, 2013. ISSN 13594311.

TURTELTAUB, S. Optimal material properties for transient problems. Structural and Multidisciplinary Optimization, v. 22, n. 2, p. 157-166, 2001. ISSN 1615147X.

VASILIEV, L. L.; KANONCHIK, L. E.; BABENKO, V. A. Thermal management of the adsorption-based vessel for hydrogenous gas storage. Journal of Engineering Physics ..., p. $12-15,2011$.

VASILIEV, L. L.; KANONCHIK, L. E.; RABETSKY, M. I. Thermally regulated cylinder for adsorption storage of a hydrogenous gas. International Journal of Heat and Mass Transfer, Elsevier Ltd, v. 71, n. 2014, p. 125-132, 2014. ISSN 00179310. 
WACHTER, A.; BIEGLER, L. T. On the implementation of an interior-point filter line-search algorithm for large-scale nonlinear programming. Mathematical Programming, v. 106, n. 1, p. 25-57, 2006. ISSN 00255610.

WANG, B. K.; VINEYARD, E. A. Adsorption refrigeration. Ashrae, v. 1, n. September, p. 14-24, 2011.

WELLER, H. G. et al. A tensorial approach to computational continuum mechanics using object orientated techniques. Computers in Physics, v. 12, n. 6, p. 620-631, 1998.

WETENHALL, B. et al. Impact of co2 impurity on co2 compression, liquefaction and transportation. Energy Procedia, v. 63, p. 2764-2778, 2014. ISSN 18766102.

WHITAKER, S. Flow in porous media i: A theoretical derivation of darcy's law. Transport in Porous Media, v. 1, n. 1, p. 3-25, 1986. ISSN 01693913.

WITHERDEN, F. D.; FARRINGTON, A. M.; VINCENT, P. E. Pyfr: An open source framework for solving advection-diffusion type problems on streaming architectures using the flux reconstruction approach. Computer Physics Communications, Elsevier B.V., v. 185, n. 11, p. 3028-3040, 2014. ISSN 00104655.

WOLAK, E.; KRASZEWSKI, S. An overview of adsorptive processes in refrigeration systems refrigerating appliances. E3S Web of Conferences, v. 10, p. 1-6, 2016. ISSN 22671242.

WOOD, G. O. Affinity coefficients of the polanyi/dubinin adsorption isotherm equations. a review with compilations and correlations. Carbon, v. 39, n. 3, p. 343-356, 2001. ISSN 00086223.

WOOD, G. O. Review and comparisons of $\mathrm{d} / \mathrm{r}$ models of equilibrium adsorption of binary mixtures of organic vapors on activated carbons. Carbon, v. 40, n. 3, p. 231-239, 2002. ISSN 00086223.

XIE, Y. M. et al. Shape and topology design for heat conduction by evolutionary structural optimization. International Journal of Heat and Mass Transfer, v. 42, n. 1999, p. 3361-3371, 1999. ISSN 0017-9310.

YONGE, D. R. Finite element modelling of multisolute activated carbon adsorption. Applied mathematical modelling, v. 16, p. 630-637, 1992.

YOON, G. H. Topological design of heat dissipating structure with forced convective heat transfer. Journal of Mechanical Science and Technology, v. 24, n. 6, p. 1225-1233, jun. 2010. ISSN 1738494X.

YOON, G. H. Topological layout design of electro-fluid-thermal-compliant actuator. Computer Methods in Applied Mechanics and Engineering, Elsevier B.V., v. 209-212, p. 28-44, fev. 2012. ISSN 00457825.

YOON, G. H.; KIM, Y. Y. The element connectivity parameterization formulation for the topology design optimization of multiphysics systems. International Journal for Numerical Methods in Engineering, v. 64, n. 12, p. 1649-1677, 2005. ISSN 00295981.

ZENG, Z.; GRIGG, R. A criterion for non-darcy flow in porous media. Transport in Porous Media, v. 63, n. 1, p. 57-69, 2006. ISSN 01693913. 


\section{Appendix A. Finite Element Coefficients}

This chapter presents the integral form of the coefficients of the FEM systems given in Chapter 4.

\section{A.1 Full Adsorption Problem}

$$
\begin{aligned}
& W(T)=\int_{\omega}[2 \pi r] \frac{M_{g}\left(\boldsymbol{\Xi}^{T} \varepsilon_{t}\right)}{R_{g}\left(\boldsymbol{\Psi}^{T} \mathbf{T}\right)} \boldsymbol{\Phi} \boldsymbol{\Phi}^{T} d \mathbf{x} \\
& S(P, T)=\int_{\omega}[2 \pi r] \frac{M_{g}\left(\boldsymbol{\Xi}^{T} \varepsilon_{t}\right)\left(\boldsymbol{\Phi}^{T} \mathbf{P}\right)}{R_{g}\left(\boldsymbol{\Psi}^{T} \mathbf{T}\right)^{2}} \boldsymbol{\Phi} \boldsymbol{\Psi}^{T} d \mathbf{x} . \\
& Z(P, T)=\int_{\omega}[2 \pi r] \frac{M_{g}\left(\boldsymbol{\Xi}^{T} \mathbf{K}_{\mathbf{s}}\right)\left(\boldsymbol{\Phi}^{T} \mathbf{P}\right)}{R_{g} \mu_{g}\left(\mathbf{\Psi}^{T} \mathbf{T}\right)} \nabla \boldsymbol{\Phi} \nabla \boldsymbol{\Phi}^{T} d \mathbf{x} \\
& -\int_{\gamma_{W}}[2 \pi r] \frac{M_{g}\left(\boldsymbol{\Xi}^{T} \mathbf{K}_{\mathbf{s}}\right)\left(\boldsymbol{\Phi}^{T} \mathbf{P}\right)}{R_{g} \mu_{g}\left(\mathbf{\Psi}^{T} \mathbf{T}\right)} \boldsymbol{\Phi} \nabla \boldsymbol{\Phi}^{T} d s . \\
& J=\int_{\omega}[2 \pi r]\left(\boldsymbol{\Xi}^{T} \varepsilon_{t}\right) \boldsymbol{\Psi} \boldsymbol{\Phi}^{T} d \mathbf{x} \\
& N(P, T, Q)=\int_{\omega}[2 \pi r] \frac{C p_{g} M_{g}\left(\boldsymbol{\Xi}^{T} \varepsilon_{\mathbf{t}}\right)}{R_{g}} \frac{\left(\boldsymbol{\Phi}^{T} \mathbf{P}\right)}{\left(\boldsymbol{\Psi}^{T} \mathbf{T}\right)} \boldsymbol{\Psi} \boldsymbol{\Psi}^{T} d \mathbf{x} \\
& +\int_{\omega}[2 \pi r] C p_{g}\left(\boldsymbol{\Xi}^{T} \mathbf{Q}\right) \Psi \Psi^{T} d \mathbf{x} \\
& +\int_{\omega}[2 \pi r]\left(1-\boldsymbol{\Xi}^{T} \varepsilon_{t}\right)\left(\boldsymbol{\Xi}^{T} \mathbf{C} \mathbf{p}_{\mathbf{s}}\right)\left(\boldsymbol{\Xi}^{T} \boldsymbol{\rho}_{s}\right) \boldsymbol{\Psi} \boldsymbol{\Psi}^{T} d \mathbf{x} .
\end{aligned}
$$




$$
\begin{aligned}
& L(P, T)=-\int_{\omega}[2 \pi r] \frac{C p_{g} M_{g}\left(\boldsymbol{\Xi}^{T} \mathbf{K}_{\mathbf{s}}\right)\left(\boldsymbol{\Phi}^{T} \mathbf{P}\right)}{R_{g} \mu_{g}\left(\boldsymbol{\Psi}^{T} \mathbf{T}\right)}\left(\nabla \boldsymbol{\Phi}^{T} \mathbf{P}\right) \nabla \boldsymbol{\Psi} \nabla \boldsymbol{\Psi}^{T} d \mathbf{x} . \\
& K=\int_{\omega}[2 \pi r]\left(\boldsymbol{\Xi}^{T} \mathbf{k}_{\mathbf{f}}\right) \nabla \boldsymbol{\Psi} \nabla \boldsymbol{\Psi}^{T} d \mathbf{x}-\int_{\gamma_{W}}[2 \pi r]\left(\boldsymbol{\Xi}^{T} \mathbf{k}_{\mathbf{f}}\right) \boldsymbol{\Psi} \nabla \boldsymbol{\Psi}^{T} d s \\
& Y_{M}=\int_{\omega}[2 \pi r] \boldsymbol{\Phi} \boldsymbol{\Xi}^{T} d x \\
& Y_{E}=\int_{\omega}-[2 \pi r]|\Delta H| \Psi \boldsymbol{\Xi}^{T} d x \\
& Y_{D}=\int_{\omega}[2 \pi r] \boldsymbol{\Xi} \boldsymbol{\Xi}^{T} d x \\
& R(T)=\int_{\omega}[2 \pi r]\left(\boldsymbol{\Xi}^{T} \mathbf{G}\right) \boldsymbol{\Xi} \boldsymbol{\Xi}^{T} d x \\
& V(P, T)=\int_{\omega}[2 \pi r]\left(\boldsymbol{\Xi}^{T} \mathbf{G}\right)\left(\boldsymbol{\Xi}^{T} \mathbf{Q}_{\mathbf{e q}}\right) \boldsymbol{\Xi} d x
\end{aligned}
$$

A.2 Adsorption Problem after Decoupling the Adsorbed Density Field

$$
\begin{gathered}
F=-\int_{\omega}[2 \pi r]\left(\boldsymbol{\Xi}^{T} \frac{\partial \mathbf{Q}}{\partial t}\right) \boldsymbol{\Phi} d \mathbf{x} . \\
H=\int_{\omega}[2 \pi r]|\Delta H|\left(\boldsymbol{\Xi}^{T} \frac{\partial \mathbf{Q}}{\partial t}\right) \mathbf{\Psi} d \mathbf{x} .
\end{gathered}
$$


A.3 Adsorption Problem after Decoupling the Pressure Field

$$
\begin{aligned}
& W=\int_{\omega}[2 \pi r]\left(\boldsymbol{\Xi}^{T} \varepsilon_{t}\right)\left(\boldsymbol{\Xi}^{T} \frac{\partial \boldsymbol{\rho}_{g}}{\partial t}\right) \boldsymbol{\Phi} \boldsymbol{\Phi}^{T} d \mathbf{x} \\
& Z=\int_{\omega}[2 \pi r] \frac{\left(\boldsymbol{\Xi}^{T} \mathbf{K}_{\mathbf{s}}\right)\left(\boldsymbol{\Xi}^{T} \boldsymbol{\rho}_{g}\right)}{\mu_{g}} \nabla \boldsymbol{\Phi} \nabla \boldsymbol{\Phi}^{T} d \mathbf{x} \\
& -\int_{\gamma_{W}}[2 \pi r] \frac{\left(\boldsymbol{\Xi}^{T} \mathbf{K}_{\mathbf{s}}\right)\left(\boldsymbol{\Xi}^{T} \boldsymbol{\rho}_{g}\right)}{\mu_{g}} \boldsymbol{\Phi} \nabla \boldsymbol{\Phi}^{T} d s . \\
& F=-\int_{\omega}[2 \pi r]\left(\boldsymbol{\Xi}^{T} \frac{\partial \mathbf{Q}}{\partial t}\right) \boldsymbol{\Phi} d \mathbf{x} \\
& N=\int_{\omega}[2 \pi r]\left(\boldsymbol{\Xi}^{T} \boldsymbol{\varepsilon}_{t}\right) C p_{g}\left(\boldsymbol{\Xi}^{T} \boldsymbol{\rho}_{g}\right) \boldsymbol{\Psi} \boldsymbol{\Psi}^{T} d \mathbf{x} \\
& +\int_{\omega}[2 \pi r]\left(1-\left(\boldsymbol{\Xi}^{T} \boldsymbol{\varepsilon}_{t}\right)\right)\left(\boldsymbol{\Xi}^{T} \boldsymbol{C} p_{s}\right)\left(\boldsymbol{\Xi}^{T} \boldsymbol{\rho}_{s}\right) \boldsymbol{\Psi} \boldsymbol{\Psi}^{T} d \mathbf{x} \\
& +\int_{\omega}[2 \pi r] C p_{g}\left(\boldsymbol{\Xi}^{T} \mathbf{Q}\right) \Psi \boldsymbol{\Psi}^{T} d \mathbf{x} . \\
& L=-\int_{\omega}[2 \pi r] \frac{C p_{g} M_{g}\left(\boldsymbol{\Xi}^{T} \mathbf{K}_{\mathbf{s}}\right)\left(\boldsymbol{\Phi}^{T} \mathbf{P}\right)}{R_{g} \mu_{g}\left(\boldsymbol{\Psi}^{T} \mathbf{T}\right)}\left(\nabla \boldsymbol{\Phi}^{T} \mathbf{P}\right) \nabla \boldsymbol{\Psi} \nabla \boldsymbol{\Psi}^{T} d \mathbf{x} . \\
& K=\int_{\omega}[2 \pi r]\left(\boldsymbol{\Xi}^{T} \mathbf{k}_{\mathbf{f}}\right) \nabla \boldsymbol{\Psi} \nabla \boldsymbol{\Psi}^{T} d \mathbf{x} \\
& -\int_{\gamma_{W}}[2 \pi r]\left(\boldsymbol{\Xi}^{T} \mathbf{k}_{\mathbf{f}}\right) \Psi \nabla \boldsymbol{\Psi}^{T} d s \\
& H=\int_{\omega}[2 \pi r]\left(\boldsymbol{\Xi}^{T} \boldsymbol{\varepsilon}_{t}\right)\left(\boldsymbol{\Xi}^{T} \frac{\partial \mathbf{P}}{\partial t}\right) \boldsymbol{\Psi} d \mathbf{x}+\int_{\omega}[2 \pi r]|\Delta H|\left(\boldsymbol{\Xi}^{T} \frac{\partial \mathbf{Q}}{\partial t}\right) \boldsymbol{\Psi} d \mathbf{x} .
\end{aligned}
$$




\section{A.4 Helmholtz Filtering}

The interpolation of the pseudo-porosities is done over a finite element by using the shape function $\xi$ as

$$
\mathrm{e}(\mathbf{x}, t) \cong \sum_{l=1}^{L} \xi_{l}(\mathbf{x}) \mathrm{e}(t)=\mathbf{\Xi}^{T} \mathbf{e}
$$

Making $v_{H}=\xi$, the FEM system is assembled based on the coefficients

$$
\begin{gathered}
Z=\int_{\omega} \boldsymbol{\Xi} \boldsymbol{\Xi}^{T} d \mathbf{x}+\int_{\omega} z^{2} \nabla \boldsymbol{\Xi} \nabla \boldsymbol{\Xi}^{T} d \mathbf{x} \\
\phi=\int_{\omega}\left(\boldsymbol{\Xi}^{T} \boldsymbol{\phi}\right) \boldsymbol{\Xi} d \mathbf{x} .
\end{gathered}
$$




\section{Appendix B. Material Properties}

Table B.1: Methane properties (SAHOO et al., 2011; SAHOO; RAMGOPAL, 2014).

\begin{tabular}{lc|rl}
\hline Property & Symbol & Value & Unit \\
\hline Specific Heat & $C p_{g}$ & 14890 & $\mathrm{~J} \mathrm{~kg}^{-1} \mathrm{~K}^{-1}$ \\
Thermal Conductivity & $k_{g}$ & $34.30 \times 10^{-3}$ & $\mathrm{~W} \mathrm{~m}^{-1} \mathrm{~K}^{-1}$ \\
Molar Mass & $M_{g}$ & $16.03 \times 10^{-3}$ & $\mathrm{~kg} \mathrm{~mol}^{-1}$ \\
Critical Pressure & $P_{c r}$ & $4.60 \times 10^{6}$ & $\mathrm{~Pa}$ \\
Boiling Point Temperature & $T_{b o i l}$ & 111.2 & $\mathrm{~K}$ \\
Critical Temperature & $T_{c r}$ & 191 & $\mathrm{~K}$ \\
Thermal Expansion of Liquefied Gases & $\alpha_{e}$ & $2.50 \times 10^{-3}$ & $\mathrm{~K}^{-1}$ \\
Density at Boiling Point & $\rho_{b o i l}$ & 422.62 & $\mathrm{~kg} \mathrm{~m}^{-3}$ \\
Viscosity & $\mu_{g}$ & $12.50 \times 10^{-6}$ & $\mathrm{~Pa} \mathrm{~s}$ \\
\hline
\end{tabular}

Table B.2: Activated Carbon properties (SAHOO et al., 2011).

\begin{tabular}{lc|rl}
\hline Property & Symbol & Value & Unit \\
\hline Bulk Specific Heat & $C p_{s}$ & 650 & $\mathrm{~J} \mathrm{~kg}^{-1} \mathrm{~K}^{-1}$ \\
Bulk Thermal Conductivity & $k_{s}$ & 0.54 & $\mathrm{~W} \mathrm{~m}^{-1} \mathrm{~K}^{-1}$ \\
Micro-Pores Dispersion & $n_{s}$ & 1.8 & \\
Specific Volume of Micro-Pores & $w$ & $0.33 \times 10^{-3}$ & $\mathrm{~m}^{3} \mathrm{~kg}^{-1}$ \\
Particle Diameter & $\emptyset_{s}$ & $1.00 \times 10^{-3}$ & $\mathrm{~m}$ \\
Macro-Porosity & $\varepsilon_{M}$ & 0.3 & \\
Micro-Porosity & $\varepsilon_{m}$ & 0.5 & \\
Bulk Density & $\rho_{s}$ & 1428.57 & $\mathrm{~kg} \mathrm{~m}$ \\
Micro-Pore Tortuosity & $\tau_{s}$ & 2.08 & \\
\hline
\end{tabular}


Table B.3: Adsorption parameters for Methane on Activated Carbon (SAHOO et al., 2011; SAHOO; RAMGOPAL, 2014).

\begin{tabular}{lc|rl}
\hline Parameter & Symbol & Value & Unit \\
\hline Effective Gas Diffusivity & $D_{0}$ & $8.00 \times 10^{-7}$ & $\mathrm{~m}^{2} \mathrm{~s}^{-1}$ \\
Characteristic Energy & $E_{0}$ & 25040 & $\mathrm{~J} \mathrm{~mol}^{-1}$ \\
Activation Energy of Adsorption & $E_{a}$ & 6000 & $\mathrm{~J} \mathrm{~mol}^{-1}$ \\
Activation Energy of Desorption & $E_{d}$ & 22000 & $\mathrm{~J} \mathrm{~mol}^{-1}$ \\
Affinity Coefficient & $\beta$ & 0.35 & \\
Isosteric Heat of Adsorption & $\Delta H$ & $-16000 \times 10^{3}$ & $\mathrm{~J} \mathrm{~mol}^{-1}$ \\
\hline
\end{tabular}

${ }^{1}$ Calculated from $E_{a}$ and $\Delta H$ values (CHRISTMANN, 1991).

Table B.4: Steel properties.

\begin{tabular}{lc|rl}
\hline Parameter & Symbol & Value & Unit \\
\hline Specific Heat & $C p_{s}$ & 470 & $\mathrm{~J} \mathrm{~kg}^{-1} \mathrm{~K}^{-1}$ \\
Thermal Conductivity & $k_{s}$ & 47 & $\mathrm{~W} \mathrm{~m}^{-1} \mathrm{~K}^{-1}$ \\
Density & $\rho_{s}$ & 7900 & $\mathrm{~kg} \mathrm{~m}^{-3}$ \\
Permeability $^{1}$ & $K_{s}$ & $1.00 \times 10^{-22}$ & $\mathrm{~m}^{2}$ \\
\hline
\end{tabular}

${ }^{1}$ Approximation adopted for representing impermeable medium. 